\title{
A Comprehensive Survey on Particle Swarm Optimization Algorithm and Its Applications
}

\author{
Yudong Zhang, ${ }^{1}$ Shuihua Wang, ${ }^{1,2}$ and Genlin Ji ${ }^{1}$ \\ ${ }^{1}$ School of Computer Science and Technology, Nanjing Normal University, Nanjing, Jiangsu 210023, China \\ ${ }^{2}$ School of Electronic Science and Engineering, Nanjing University, Nanjing, Jiangsu 210046, China \\ Correspondence should be addressed to Yudong Zhang; zhangyudongnuaa@gmail.com
}

Received 15 December 2014; Revised 12 February 2015; Accepted 12 February 2015

Academic Editor: Shuming Wang

Copyright (c) 2015 Yudong Zhang et al. This is an open access article distributed under the Creative Commons Attribution License, which permits unrestricted use, distribution, and reproduction in any medium, provided the original work is properly cited.

\begin{abstract}
Particle swarm optimization (PSO) is a heuristic global optimization method, proposed originally by Kennedy and Eberhart in 1995. It is now one of the most commonly used optimization techniques. This survey presented a comprehensive investigation of PSO. On one hand, we provided advances with PSO, including its modifications (including quantum-behaved PSO, bare-bones PSO, chaotic PSO, and fuzzy PSO), population topology (as fully connected, von Neumann, ring, star, random, etc.), hybridization (with genetic algorithm, simulated annealing, Tabu search, artificial immune system, ant colony algorithm, artificial bee colony, differential evolution, harmonic search, and biogeography-based optimization), extensions (to multiobjective, constrained, discrete, and binary optimization), theoretical analysis (parameter selection and tuning, and convergence analysis), and parallel implementation (in multicore, multiprocessor, GPU, and cloud computing forms). On the other hand, we offered a survey on applications of PSO to the following eight fields: electrical and electronic engineering, automation control systems, communication theory, operations research, mechanical engineering, fuel and energy, medicine, chemistry, and biology. It is hoped that this survey would be beneficial for the researchers studying PSO algorithms.
\end{abstract}

\section{Introduction}

Artificial intelligence (AI) is the intelligence exhibited by machines. It is defined as "the study and design of intelligent agents" [1], where an intelligent agent represents a system that perceives its environment and takes action that maximizes its success chance. AI research is highly technical and specialized and is deeply divided into subfields that often fail to communicate with each other. Currently popular approaches of AI include traditional statistical methods [2], traditional symbolic AI, and computational intelligence (CI) [3]. CI is a fairly new research area. It is a set of nature-inspired computational methodologies and approaches to address complex real-world problems to which traditional approaches are ineffective or infeasible. CI includes artificial neural network (ANN), fuzzy logic, and evolutionary computation (EC).

Swarm intelligence (SI) is a part of EC. It researches the collective behavior of decentralized, self-organized systems, natural or artificial. Typical SI systems consist of a population of simple agents or boids interacting locally with one another and with their environment. The inspiration often comes from nature, especially biological systems [4].

The agents in a SI system follow very simple rules. There is no centralized control structure dictating how individual agents should behave. The agents' real behaviors are local, and to a certain degree random; however, interactions between such agents lead to the emergence of "intelligent" global behavior, which is unknown to the individual agents. Wellknown examples of SI include ant colonies, bird flocking, animal herding, bacterial growth, and fish schooling.

Dorigo [5] proposed an ant colony optimization (ACO) method based on ant colony. Kennedy and Eberhart [6] proposed a particle swarm optimization (PSO) method based on bird flocking. Those are two most famous SI-based optimization algorithms. In addition to them, scholars have shown great interest in proposing new intelligent approaches. Storn and Price [7] proposed a differential evolution (DE). Müller et al. [8] and Passino [9] proposed the bacterial foraging optimization (BFO), inspired by the group foraging behavior of bacteria such as E. coli and M. xanthus. Karaboga 


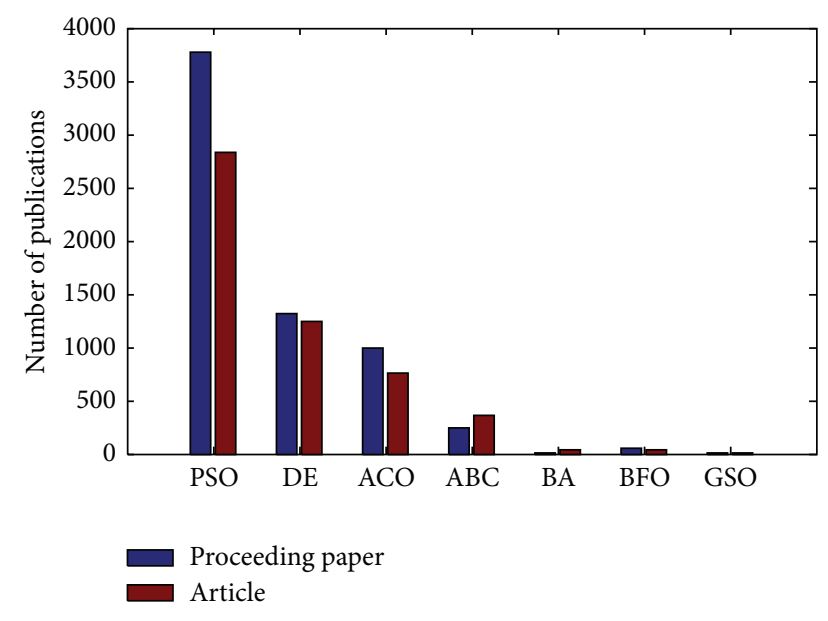

FIgURE 1: Number of all publications w.r.t. SI-based algorithms.

and Basturk [10] proposed artificial bee colony (ABC), which simulates the foraging behavior of honey bees. Krishnanand and Ghose [11] proposed glowworm swarm optimization (GSO) method, the agents in which are thought of as glowworms that carry a luminescence quantity called luciferin along with them. Yang [12] proposed a bat algorithm (BA), inspired by the echolocation behavior of microbats.

The distribution of all publications and publication per year w.r.t. SI-based algorithms is presented in Figures 1 and 2 , respectively. As seen from the figures, the number of total publications related to PSO is even higher than the sum of six other algorithms, and the number of publication per year related to PSO is the highest among all seven SI-based algorithms. This suggests PSO is the most prevalent SI-based optimization algorithms. Therefore, we center this review on PSO.

Several public websites related to PSO (Table 1) were set up [13], dedicated to share the codes, ideas, and latest advances on PSO. There are several types of source codes, written in different programming languages, in those websites. In addition, many publications about PSO and its applications were presented.

This work first checked the coherency of PSO with principles required by SI. Second, we reviewed the studies on advances of PSO. Third, various applications of PSO is given. Finally, we conclude the paper by summarizing the improvements and analyzing potential research directions. This survey was carried out mainly by examining the "Web of Science Core Collection" database. In addition, IEEE Explorer and Google Scholar were also used.

\section{Particle Swarm Optimization: PSO Approach}

2.1. Features of Self-Organization. Self-organization is a key feature of SI system. It is a process where global order or coordination arises out of the local interactions between the components of an initially disordered system. This process is

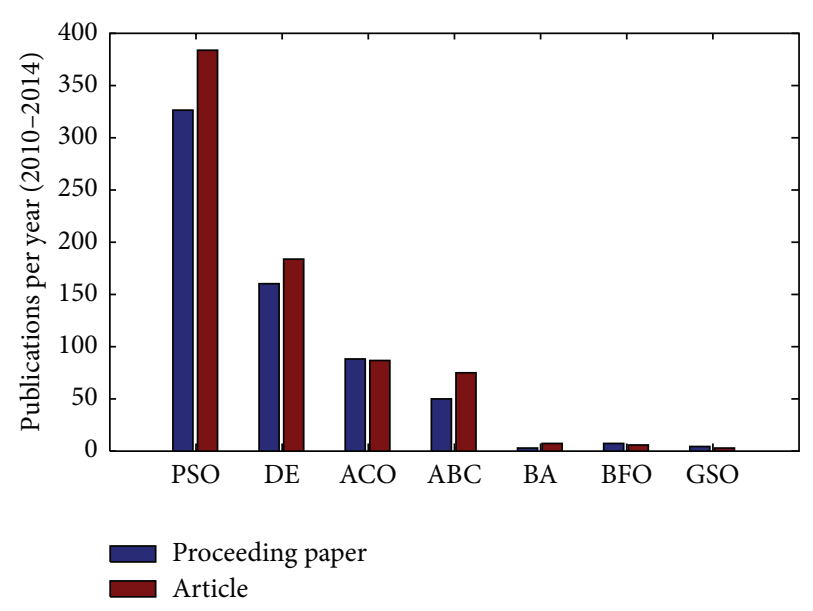

Figure 2: Publication per year (2010-2014) w.r.t. SI-based algorithms.

spontaneous; that is, it is not controlled by any agent inside or outside of the system. Bonabeau et al. [14] interpreted the self-organization in swarms through three basic ingredients as follows.

(1) Strong dynamical nonlinearity (often involving positive and negative feedback): positive feedback helps promote the creation of convenient structures, while negative feedback counterbalances positive feedback and helps to stabilize the collective pattern.

(2) Balance of exploitation and exploration: SI identifies a suitable balance to provide a valuable mean artificial creativity approach.

(3) Multiple interactions: agents in the swarm use information coming from neighbor agents so that the information spreads throughout the network.

2.2. Features of SI. In addition, Millonas [15] in Santa Fe Institute proposed five principles that SI must satisfy. They are proximity principle, quality principle, diverse response principle, stability principle, and adaptability principle. Their meanings are listed in Table 2.

2.3. Algorithmic Structure of Standard PSO. PSO performs searching via a swarm of particles that updates from iteration to iteration. To seek the optimal solution, each particle moves in the direction to its previously best (pbest) position and the global best (gbest) position in the swarm [16]. One has

$$
\begin{aligned}
& \text { pbest }(i, t)=\underset{k=1, \ldots, t}{\arg \min }\left[f\left(P_{i}(k)\right)\right], \quad i \in\left\{1,2, \ldots, N_{P}\right\}, \\
& \text { gbest }(t)=\underset{\substack{i=1, \ldots, N_{P} \\
k=1, \ldots, t}}{\arg \min }\left[f\left(P_{i}(k)\right)\right],
\end{aligned}
$$

where $i$ denotes the particle index, $N_{P}$ the total number of particles, $t$ the current iteration number, $f$ the fitness 
TABle 1: Public websites of PSO.

\begin{tabular}{|c|c|}
\hline Website name & URL \\
\hline Particle swarm optimization & http://www.swarmintelligence.org/ \\
\hline Particle swarm optimization & http://www.scholarpedia.org/article/Particle_swarm_optimization \\
\hline Particle swarm central & http://www.particleswarm.info/ \\
\hline PSO toolbox & http://psotoolbox.sourceforge.net/ \\
\hline PSO visualization & http://www.projectcomputing.com/resources/psovis/ \\
\hline Particle swarm optimization toolbox & http://atoms.scilab.org/toolboxes/PSO \\
\hline
\end{tabular}

TABLE 2: Five basic principles of SI.

\begin{tabular}{ll}
\hline Principle & Definition \\
\hline Proximity principle & The swarm should be able to do simple space and time computations \\
Quality principle & The swarm should be able to respond to quality factors in the environment \\
Diverse response principle & The swarm should not commit its activities along excessively narrow channels \\
Stability principle & The swarm should not change its mode of behavior every time the environment changes \\
Adaptability principle & The swarm should be able to change its behavior mode when it is worth the computational price \\
\hline
\end{tabular}

function, and $P$ the position. The velocity $V$ and position $P$ of particles are updated by the following equations:

$$
\begin{gathered}
V_{i}(t+1)=\omega V_{i}(t)+c_{1} r_{1}\left(\text { pbest }(i, t)-P_{i}(t)\right) \\
+c_{2} r_{2}\left(\text { gbest }(t)-P_{i}(t)\right) \\
P_{i}(t+1)=P_{i}(t)+V_{i}(t+1)
\end{gathered}
$$

where $V$ denotes the velocity, $\omega$ is the inertia weight used to balance the global exploration and local exploitation, $r_{1}$ and $r_{2}$ are uniformly distributed random variables within range $[0,1]$, and $c_{1}$ and $c_{2}$ are positive constant parameters called "acceleration coefficients."

It is common to set an upper bound for the velocity parameter. "Velocity clamping" [17] was used as a way to limit particles flying out of the search space. Another method is the "constriction coefficient" strategy, proposed by Clerc and Kennedy [18], as an outcome of a theoretical analysis of swarm dynamic, in which the velocities are constricted too.

The first part of formula (2), known as "inertia," represents the previous velocity, which provides the necessary momentum for particles to roam across the search space. The second part, known as the "cognitive" component, represents the individual particle thinking of each particle. It encourages the particles to move toward their own best positions found so far. The third part, the "cooperation" component, represents the collaborative effect of the particles to find the global optimal solution [19].

2.4. Pseudocode of PSO. Let $f: \mathbb{R}^{N} \rightarrow \mathbb{R}$ be the cost function to be minimized. The function takes a candidate solution of a vector of $N_{P}$ real numbers and produces a real number as output that indicates the cost function value. The gradient of $f$ is either unknown or hard to calculate. The goal is to find the global minimal $x^{*}$ (Pseudocode 1 ).

\section{Studies on PSO}

In this review, we center in reporting the advances on PSO in the form of formal publications. We divide advances into following six aspects:

(i) modifications of PSO, including quantum-behaved PSO, bare-bones PSO, chaotic PSO, fuzzy PSO, PSOTVAC, opposition-based PSO, topology, and other slight modifications,

(ii) hybridization of PSO with other metaheuristic methods, including genetic algorithm (GA), artificial immune system (AIS), Tabu search (TS), ACO, simulated annealing (SA), ABC, DE, biogeography-based optimization (BBO), and harmonic search (HS),

(iii) extensions of PSO to other optimization fields, including multiobjective, constrained, discrete, and binary optimization,

(iv) theoretical analysis of PSO, parameter selection and convergence analysis.

(v) parallel implementation of PSO, including multicore, GPU computing, and cloud computing.

\subsection{Modifications}

3.1.1. QPSO. Some researchers proposed quantum-behaved PSO (QPSO), which was motivated by concepts from quantum mechanics. For example, Jau et al. [20] proposed a modified QPSO, which used a high breakdown regression estimator and a least-trimmed-squares method to eliminate the influence caused by observations containing outliers. Besides, elitist crossover of GA and adaptive decay of SA are used for conquering premature and controlling search policy. Jamalipour et al. [21] presented QPSO with differential mutation operator (QPSO-DM) for optimizing WWER-1000 core fuel management. The results showed that QPSODM performs better than the others. Bagheri et al. [22] used QPSO to forecast financial time series, especially for 
Step 1. Initialization

For each particle $i=1, \ldots, N_{p}$, do

(a) Initialize the particle's position with a uniformly distribution as $P_{i}(0) \sim U(\mathrm{LB}, \mathrm{UB})$, where $\mathrm{LB}$ and $\mathrm{UB}$ represent the lower and upper bounds of the search space

(b) Initialize pbest to its initial position: pbest $(i, 0)=P_{i}(0)$.

(c) Initialize gbest to the minimal value of the swarm: gbest $(0)=\operatorname{argmin} f\left[P_{i}(0)\right]$.

(d) Initialize velocity: $V_{i} \sim U(-|\mathrm{UB}-\mathrm{LB}|,|\mathrm{UB}-\mathrm{LB}|)$.

Step 2. Repeat until a termination criteria is met

For each particle $i=1, \ldots, N_{P}$, do

(a) Pick random numbers: $r_{1}, r_{2} \sim U(0,1)$.

(b) Update particle's velocity. See formula (2).

(c) Update particle's position. See formula (3).

(d) If $f\left[P_{i}(t)\right]<f[$ pbest $(i, t)]$, do

(i) Update the best known position of particle $i$ : pbest $(i, t)=P_{i}(t)$.

(ii) If $f\left[P_{i}(t)\right]<f[g b e s t(t)]$, update the swarm's best known position: $g$ best $(t)=P_{i}(t)$.

(e) $t \leftarrow(t+1)$;

Step 3. Output gbest $(t)$ that holds the best found solution.

Pseudocode 1: A standard PSO.

the foreign exchange market. Tang et al. [23] proposed an improved QPSO algorithm for continuous nonlinear largescale problems based on memetic algorithm and memory mechanism. The memetic algorithm was used to make all particles gain some experience through a local search before being involved in the evolutionary process, and the memory mechanism was used to introduce a "bird kingdom" with memory capacity, both of which can improve the global search ability of the algorithm. Davoodi et al. [24] proposed a new approach, based on a hybrid algorithm combining of improved QPSO and simplex algorithms. QPSO was the main optimizer of algorithm, which can give a good direction to the optimal global region. Nelder-Mead simplex method was used as a local search to fine-tune the obtained solution from QPSO. Li and Xiao [25] proposed an encoding approach based on qubits described on the Bloch sphere. Each particle contained three groups of Bloch coordinates of qubits, and all three groups of coordinates were regarded as approximate solutions describing the optimization result. Particles were updated using the rotation of qubits about an axis on the Bloch sphere. Yumin and Li [26] integrated artificial fish swarm to QPSO and used adaptive parameters to avoid premature. Jia et al. [27] proposed an enhanced QPSO based on GA to realize a synchronous optimization of sensor array and classifier. Gholizadeh and Moghadas [28] proposed an improved QPSO metaheuristic algorithm to implement performance-based optimum design process. Two numerical examples were presented to illustrate the efficiency of the presented method.

3.1.2. BBPSO. The bare-bones PSO (BBPSO) [29] is a version of the PSO algorithm in which the velocity and position update rules are substituted by a procedure that samples a parametric probability density function. Zhang et al. [30] used both mutation and crossover operators of DE algorithm to modify original BBPSO in order to update certain particles in the population. The performance of the resulting algorithm was tested on 10 benchmark functions and applied to 16 vapor-liquid equilibrium problems. Zhang et al. [31] analyzed the sampling distribution in BBPSO, based on which they propose an adaptive version inspired by the cloud model, which adaptively produced a different standard deviation of the Gaussian sampling for each particle according to the evolutionary state in the swarm, which provided an adaptive balance between exploitation and exploration on different objective functions. Zhang et al. [32] proposed three global optimization algorithms for phase and chemical equilibrium calculations, which played a significant role in the simulation, design, and optimization of separation processes in chemical engineering. The proposed algorithms were unified BBPSO (UBBPSO), integrated DE (IDE), and IDE without Tabu list and radius (IDE_N). Zhang et al. [33] proposed a new bare-bones multiobjective PSO algorithm to solve the environmental/EDPs. The algorithm had three distinctive features: a particle updating strategy that did not require tuning up control parameters, a mutation operator with action range varying over time to expand the search capability, and an approach based on particle diversity to update the global particle leaders. Blackwell [34] formulated the dynamic update rule of PSO as a secondorder stochastic difference equation. General relations were derived for search focus, search spread, and swarm stability at stagnation. The relations were applied to three particular PSO implementations: the standard PSO, a PSO with discrete recombination, and the BBPSO. Wang et al. [35] proposed a novel hybrid algorithm, called SM-MBBPSO, based on the Nelder-Mead simplex method (SM) and a modified BBPSO (MBBPSO). A new strategy based on $K$-means clustering was proposed to combine the powerful global search capability of MBBPSO and the high accurate local search capability of SM. This made the proposed algorithm achieve a nice balance between exploitation and exploration capability. Meanwhile, an adaptive reinitialization strategy on inactive particles was proposed to help the swarm get away from local optimal positions. Jiang and Wang [36] used 
cooperative coevolution (CC) to improve the performance of PSO on clustering high-dimensional datasets. Based on CC framework, the original partitional clustering problem was first decomposed to several subproblems, each of which was then evolved by an optimizer independently. BBPSO was employed as the optimizer to solve each subproblem cooperatively. In addition, a new centroid-based encoding schema was designed for each particle, and the Chernoff bounds were applied to decide a proper population size. Liu et al. [37] proposed a novel disruption strategy, originating from astrophysics, to shift the abilities between exploration and exploitation during the search process, with the aim of enhancing population diversity and speeding up convergence rate of BBPSO. They researched the distribution and diversity on the proposed disruption operator and illustrated the position relationship between the original and disrupted position. Campos et al. [38] proposed a variant of BBPSO with scale matrix adaptation (SMA), SMA-BBPSO for short reference, to address the drawback of premature convergence and improve the performance of the BBPSO. The position of a particle was selected from a multivariate $t$-distribution with a rule for adaptation of its scale matrix. The multivariate $t$-distribution was used in its hierarchical form, as a scale mixture of normal distributions. In addition, the approach included the normal distribution as a particular case. As a consequence, the $t$-distribution could be applied during the optimization process by maintaining the proper balance between exploration and exploitation. Zhang et al. [39] proposed a binary BBPSO to find optimal feature subset, which was a useful preprocessing technique for solving classification problems. In this algorithm, a reinforced memory strategy was designed to update the local leaders of particles for avoiding the degradation of outstanding genes in the particles, and a uniform combination was proposed to balance the local exploitation and the global exploration of algorithm.

3.1.3. CPSO. Concepts related to chaos theory have been integrated with PSO to improve its performance. This type of PSO variant is called chaotic PSO (CPSO). Chuang et al. [40] introduced chaotic maps into catfish particle swarm optimization. The proposed method increased the search capability via the chaos approach. Zhang and Wu [41] proposed adaptive CPSO (ACPSO) to train the weights/biases of two-hidden-layer forward neural network in order to develop a hybrid crop classifier for polarimetric synthetic aperture radar images. Dai et al. [42] proposed a novel adaptive chaotic embedded PSO (ACEPSO) and applied it in wavelet parameters estimation. ACEPSO embedded chaotic variables in standard PSO and adjusted parameters nonlinearly and adaptively. It also estimated particles whether being focusing or discrete by judging the population fitness variance of particle swarm and average distance amongst points; then chaotic researching was applied to escaping from premature convergence. Li et al. [43] proposed a novel chaotic particle swarm fuzzy clustering (CPSFC) algorithm based on a new CPSO and gradient method. The new CPSO algorithm is the combination of adaptive inertia weight factor (AIWF) and iterative chaotic map with infinite collapses (ICMIC) based chaotic local search. The CPSFC algorithm utilized CPSO to search the fuzzy clustering model, exploiting the searching capability of fuzzy c-means (FCM) and avoiding its major limitation of getting stuck at locally optimal values. Meanwhile, gradient operator is adopted to accelerate convergence of the proposed algorithm. Wu et al. [44] proposed a novel support vector regression machine (SVRM) and then developed a CPSO to estimate its unknown parameters. The results of two experiments demonstrate the feasibility of this approach. Zhang et al. [45] proposed a fitness-scaling adaptive chaotic PSO (FAC-PSO) approach as a fast and robust approach for the task of path planning of unmanned combat aerial vehicle (UCAV). The FAC-PSO employed the fitness-scaling method, the adaptive parameter mechanism, and the chaotic theory. Experiments showed that the FAC-PSO was more robust and cost less time than elite GA with migration, SA, and chaotic ABC. Zhang et al. [46] combined CPSO with K2 algorithm and applied the method to Bayesian network structure learning. Yang et al. [47] applied PSO with double-bottom chaotic maps (DBMPSO) in order to assist statistical methods in the analysis of associated variations to disease susceptibility. Analysis results supported that the proposed DBM-PSO could identify good models and provided higher chi-square values than conventional PSO. Son [48] used CPSO to optimize municipal solid waste collection in GIS based environments and took Danang city, Vietnam, as a case study. He et al. [49] proposed a novel hybrid model combining ANN and CPSO to improve forecast accuracy. The proposed model was found to perform better for fine particles than for coarse particles. Zeng and Sun [50] combined classical PSO with a chaotic mechanism, time-variant acceleration coefficients, and a selfadaptive mutation scheme to prevent premature convergence and improve solution quality. Multiple efficient constraint handling strategies were employed to deal with complex constraints. Pluhacek et al. [51] utilized different chaotic systems as pseudorandom number generators (PRNGs) for velocity calculation in the PSO algorithm. Two chaos-based PRNGs were used alternately within one run of the PSO algorithm and dynamically switched over when a certain criterion was met.

3.1.4. FPSO. In order to make PSO more powerful, it was combined with fuzzy sets theory. This type of PSO variant is called fuzzy PSO (FPSO). Juang et al. [52] proposed an adaptive FPSO (AFPSO) algorithm. The proposed AFPSO utilized fuzzy set theory to adjust PSO acceleration coefficients adaptively and was thereby able to improve the accuracy and efficiency of searches. Incorporating this algorithm with quadratic interpolation and crossover operator further enhanced the global searching capability to form a new variant called AFPSO-Q1. Alfi and Fateh [53] presented a novel improved FPSO (IFPSO) algorithm to the intelligent identification and control of a dynamic system. The proposed algorithm estimated optimally the parameters of system and controller by minimizing the mean of squared errors. The PSO was enhanced intelligently by using a fuzzy inertia weight to rationally balance the global and local exploitation 
abilities. Every particle dynamically adjusted inertia weight according to particles best memories using a nonlinear fuzzy model. Yang et al. [54] proposed a novel FPSO algorithm based on fuzzy velocity updating strategy in order to optimize the machining parameters. The proposed FPSO algorithm achieved good results on few benchmark problems and obtained significant improvements on two illustrative case studies of multipass face milling. Norouzzadeh et al. [55] proposed a light adaptive PSO, which was a novel method that used a fuzzy control system to conduct the standard algorithm. The suggested method used two adjunct operators along with the fuzzy system in order to improve the base algorithm on global optimization problems. Robati et al. [56] studied an extension of PSO algorithm, the balanced fuzzy PSO algorithm, which was used for fundamental optimization problem entitled traveling salesman problem (TSP). Khan and Engelbrecht [57] presented a multiobjective PSO to efficiently solve the distributed local area networks (DLAN) topology design problem. Fuzzy logic was incorporated in the PSO algorithm to handle the multiobjective nature of the problem. Unified "And-Or" operator was used to aggregate the objectives. Results suggest that the fuzzy PSO is a suitable algorithm for solving the DLAN topology design problem. Galzina et al. [58] described the application of a hybrid of fuzzy logic and PSO in order to achieve suboptimal solutions for flow-shop scheduling problem. They named the proposed method adaptive fuzzy PSO. Nafar et al. [59] proposed a combination of FPSO and ACO method to estimate the parameters of Metal Oxide Surge Arrester (MOSA) models. The proposed method was named modified FPSO (MFPSO). The inertia weight was tuned by using fuzzy rules. Aminian and Teshnehlab [60] introduced a novel FPSO method in which the inertia weight as well as the cognitive and social coefficients was adjusted for each particle separately according to the information coming from a fuzzy logic controller. Chai et al. [61] used Hilbert-Huang transform for the features extractor and FPSO with cross mutated-based ANN (FPSOCM-ANN), for the classification of a three-class mental task-based brain-computer interface (BCI).

3.1.5. PSOTVAC. PSO with time-varying acceleration coefficients (TVAC) was proposed to further improve the performance of standard PSO. The new variant was termed PSOTVAC. Cai et al. [62] considered linear automation strategy may not work well in many cases. Therefore, a new variant, predicted modified PSO with time-varying accelerator coefficients, was proposed, in which the social and cognitive learning factors were adjusted according to a predefined predicted velocity index. The mechanism lied in that the large cognitive coefficient provided a large local search capability, whereas the small one employed a large global search capability. Chaturvedi et al. [63] employed PSOTVAC to solve the practical economic dispatch problem (EDP). TVAC here was to efficiently control the local and global search so that premature convergence was avoided and global solutions were achieved. Boonyaritdachochai et al. [64] proposed an optimal congestion management approach in a deregulated electricity market using PSOTVAC. Initially, the values of generator sensitivity were used to select redispatched generators. PSOTVAC was used to determine the minimum redispatch cost. Sun et al. [65] presented a comparative analysis of PSO, self-organizing hierarchical PSO (HPSO), and self-organizing hierarchical PSO with timevarying acceleration coefficients (HPSO-TVAC) for data clustering. They found that the HPSO and the HPSO-TVAC algorithms had better performance than the PSO algorithm in most cases, and all the clustering algorithms using PSO had good performance for large-scale data and high-dimensional data, over six well-known benchmarks. Abedinia et al. [66] presented an efficient approach for solving economic load dispatch (ELD) problems in different test power systems using PSOTVAC. Accordingly, for practical operation, many realistic constraints as ramp rate limits, generation limitation, prohibited operating zone, transmission loss, and nonlinear cost functions were considered. Mohammadi-Ivatloo et al. [67] presented a novel heuristic algorithm for solving EDP by employing iteration PSO with time-varying acceleration coefficients (IPSO-TVAC) method. EDP may even be more complicated if transmission losses were taken into account. Numerical results showed that the IPSO-TVAC method had a good convergence property. Mohammadi-Ivatloo et al. [68] implemented a novel time-varying acceleration coefficients PSO (TVAC-PSO) algorithm to solve combined heat and power ED (CHPED). The acceleration coefficients in PSO algorithm were varied adaptively during iterations to improve solution quality of original PSO and avoid premature convergence. Pookpunt and Ongsakul [69] proposed a binary PSO (BPSO) with TVAC for solving optimal placement of wind turbines within a wind farm. The objective was to extract the maximum turbine power output in a minimum investment cost within a wind farm. The BPSO-TVAC algorithm was applied to 100-square-cell test site considering uniform wind and nonuniform wind speed with variable direction characteristics. Linear wake model was used to calculate downstream wind speed. Abedinia et al. [70] presented a hybrid PSO with time-varying acceleration coefficients (HPSOTVAC) and bacteria foraging algorithm (BFA) for solving a complex ELD problem. The effectiveness of the proposed HPSOTVAC/BFA was tested in 6-, 15-, and 40unit generating systems. Abdullah et al. [71] proposed a modified PSO with TVAC (MPSO-TVAC) for solving ELD problem. To improve the solution quality and robustness of PSO algorithm, a new best neighbor particle called " $r b e s t$ " was proposed. The rbest provided extra information for each particle that was randomly selected from other best particles in order to diversify the movement of particle and avoid premature convergence. Chih et al. [72] adapted standard PSO and proposed two novel PSO algorithms, namely, the binary PSO with TVAC (BPSOTVAC) and the chaotic binary PSO with TVAC (CBPSOTVAC), to solve the multidimensional knapsack problem (MKP). The results showed that the proposed algorithms were superior over the other methods according to success rate, mean absolute deviation, mean absolute percentage error, least error, and standard deviation. 
3.1.6. OPSO. Opposition-based learning (OBL) theory was integrated with PSO, and the new variant was dubbed opposition-based PSO (OPSO). Dhahri and Alimi [73] proposed the OPSO using the concept of opposite number to create a new population during the learning process. They combined OPSO with BBFNN. The results showed that the OPSO-BBFNN produced a better generalization performance. Wang et al. [74] presented an enhanced PSO algorithm called GOPSO, which employed generalized OBL (GOBL) and Cauchy mutation. GOBL provided a faster convergence and the Cauchy mutation with a long tail helped trapped particles escape from local optima. Dong et al. [75] proposed an evolutionary circle detection method based on a novel chaotic hybrid algorithm (CHA). The method combined the strengths of PSO, GA, and chaotic dynamics and involved the standard velocity and position update rules of PSOs, with the ideas of selection, crossover, and mutation from GA. The OBL was employed in CHA for population initialization. In addition, the notion of species was introduced into the proposed CHA to enhance its performance in solving multimodal problems. Gao et al. [76] proposed a novel PSO called CSPSO to improve the performance of PSO on complex multimodal problems. Specifically, a stochastic search technique was used to execute the exploration in PSO. In addition, to enhance the global convergence, when producing the initial population, both opposition-based learning method and chaotic maps were employed. The numerical simulation and comparisons with some typical existing algorithms demonstrated the superiority of the proposed algorithm. Khan et al. [77] presented a new discrete PSO approach to induce rules from discrete data. The proposed algorithm, called Opposition-Based Natural Discrete PSO (ONDPSO), initialized its population by taking into account the discrete nature of the data. Particles were encoded using a Natural Encoding scheme. Each member of the population updated its position iteratively based on a newly designed position update rule. OBL was implemented in the optimization process. The encoding scheme and position update rule used by the algorithm allowed individual terms corresponding to different attributes within the rule's antecedent to be a disjunction of the values of those attributes. Kaucic [78] presented a multistart PSO algorithm for the global optimization of a function subject to bound constraints. The procedure consisted of three main steps. In the initialization phase, an OBL strategy was performed to improve the search efficiency. Then a variant of the adaptive velocity based on the differential operator enhanced the optimization ability of the particles. Finally, a reinitialization strategy based on two diversity measures for the swarm was acted in order to avoid premature convergence and stagnation. Dai et al. [79] established a mathematical model to study the motions of ships in order to control them effectively. They proposed an algorithm based on PSO and the OBL theory, known as the opposition-based particle swarm optimization (OPSO). Muñoz et al. [80] described how adequate hardware implementations of the PSO algorithm can be useful for realtime adaptation of mobile robot controllers. For achieving this goal, they proposed a new architecture, which was based on an FPGA implementation of the OBL approach applied to the PSO and which explored the intrinsic parallelism of this algorithm in order to adjust the weights of a neural robot controller in real time according to desired behaviors.

3.1.7. SPSO. In contrast, some researchers objected to those researches that made PSO more and more complex, and they tended to simplify standard PSO without impairing its performance, with the aim of reducing computation time, improving convergence performance, or making it easier to implement. For example, Guochu [81] divided the swarm into three categories denoted as better particles, ordinary particles, and the worst particles, according to the fitness values. These three types of particles evolved dynamically according to three corresponding kinds of simplified algorithm models. The results showed that simplified PSO (SPSO) had better optimization performance than other improved PSOs. Pedersen and Chipperfield [82] simplified PSO method in order to increase its adaptability and used an overlaid metaoptimizer for efficiently tuning behavior parameters. The simplified version was dubbed Many Optimizing Liaisons (MOL). Experiments showed that MOL had comparable performance with PSO. Martins et al. [83] proposed a simplified PSO, which allowed saving some computational effort and obtained a considerable performance in the optimization of nonlinear functions. The method was tested by four nonlinear benchmark functions: Sphere, Schwefel, Schaffer, and Ackley. Panda et al. [84] presented the design and performance analysis of proportional-integral derivate (PID) controller for an automatic voltage regulator system using MOL. The superiority of MOL was shown by comparing the results with ABC, PSO, and DE. Vastrakar and Padhy [85] proposed a simplified PSO with proportional-integral proportionalderivative (PI-PD) controller for unstable processes. Complete search space was divided into small subsearch spaces. In each subspace, they calculated the global minima and local minima and took the minimum of all, that is, minimum of complete search space. Yeh [86] proposed a parameter-free simplified swarm optimization to adjust the weights in ANNs.

3.1.8. Topology. The premature of PSO can be avoided by not using the entire swarm's best known position gbest but just the best position of the local area around the particle that is moved. In such case, the PSO variant is said to be local best lbest. Further, suppose there is an information link between each particle and its neighbors, and the set of those links builds a graph, which is called the topology of PSO variant. Scholars have carried out numerous researches in this field. Wang and Watada [87] proposed a hybrid mutationneighborhood-based PSO (MN-PSO) which comprised the approximation algorithm to search for the approximate optimal solution. Jiang et al. [88] proposed PSO with age-group topology (PSOAG), a novel age-based PSO. They presented a new concept of age to measure the search ability of each particle in local area. To keep population diversity during searching, particles were separated to different age-groups by their age. Particles in each age-group could only select the ones in younger groups or their own groups as their neighborhoods. To allow search escape from local optima, 
the aging particles were regularly replaced by new and randomly generated ones. In addition, an age-group based parameter setting method was designed, where particles in different age-groups had different parameters to accelerate convergence. Marinakis and Marinaki [89] introduced a new algorithmic nature-inspired approach that used PSO with different neighborhood topologies, for successfully solving one of the most computationally complex problems, the permutation flow-shop scheduling problem (PFSP). The proposed algorithm combined a PSO algorithm, the variable neighborhood search strategy, and a path relinking strategy. Rada-Vilela et al. [90] presented a performance study on the effect of synchronicity in communications and neighborhood size in PSO on large-scale optimization problems. The algorithms under study were the Synchronous PSO (S-PSO), the Asynchronous PSO (A-PSO), and the recently proposed Random Asynchronous PSO (RA-PSO). Wang et al. [91] proposed a hybrid PSO algorithm called DNSPSO, which employed a diversity enhancing mechanism and neighborhood search strategies to achieve a trade-off between exploration and exploitation abilities. Comparison results showed that DNSPSO obtained a promising performance on the majority of the test problems. Fu et al. [92] proposed a new QPSO algorithm called NQPSO, in which one local and one global neighborhood search strategies were utilized to balance exploitation and exploration. Moreover, a concept of opposition-based learning was employed for population initialization. Computational results showed that the proposed approach outperformed some similar QPSO algorithms and five other state-of-theart PSO variants. Ni and Deng [93] proposed to use random topology and analyzed its performance. The relationship between population topology and the performance of PSO was also explored from the perspective of graph theory characteristics in population topologies. Further, in logistic dynamic particle optimization, an extensive simulation study was presented to discuss the effectiveness of the random topology and the design strategies of population topology. Beheshti et al. [94] proposed an improved PSO scheme called fusion global-local-topology PSO (FGLT-PSO). The algorithm employed both global and local topologies in PSO to jump out of the local optima. The experimental results showed that the proposed method improved the performance of PSO algorithm in terms of solution accuracy and convergence speed. Lim and Isa [95] proposed PSO with increasing topology connectivity (PSO-ITC) to solve unconstrained single-objective optimization problems with continuous search space. An ITC module was developed to achieve better control of exploration/exploitation searches by linearly increasing the particle's topology connectivity with time as well as performing the shuffling mechanism. Furthermore, they introduced a new learning framework that consisted of a new velocity update mechanism and a new neighborhood search operator that aimed at enhancing the algorithm's searching performance. Kalayci and Gupta [96] proposed a new approach based on the PSO algorithm with a neighborhood-based mutation operator to solve the sequence-dependent disassembly line balancing problem.
3.1.9. Other Modifications. Some researchers make tentative research on improving the optimization performance of PSO by other efficient strategies. For example, Chuang et al. [97] proposed a novel catfish PSO, the mechanism of which is dependent on the incorporation of a catfish particle into the linearly decreasing weight particle swarm optimization. Unlike other ordinary particles, the catfish particles initialized a new search from the extreme points of the search space when the gbest fitness value had not been changed for a given time, which resulted in further opportunities to find better solutions for the swarm by guiding the whole swarm to promising new regions of the search space and accelerating convergence. Shi and Liu [98] proposed a hybrid improved $\mathrm{PSO}$, in which chaos initialization was introduced to improve the population diversity, and adaptive parameters' control strategy was employed to make it independent from specific problem. Besides, novel acceptance policy based on Metropolis rule was taken to guarantee the convergence of the algorithm. Zhang et al. [99] proposed a new adaptive PSO (APSO) that could dynamically follow the frequently changing market demand and supply in each trading interval. A numerical example served to illustrate the essential features of the approach. Liu et al. [100] proposed an improved cooperative PSO to solve both the local extrema and the pseudominimum problem, with the aim of solving production scheduling more efficiently. The results showed that the convergent speed and solution quality of the improved cooperative PSO preceded the other two efficient algorithms. Shen et al. [101] presented a correlation PSO model in which a novel correlative strategy was used to process the personal experience and sharing experience. The relational expression between the correlation coefficient and population diversity was developed through theoretical analysis. They found that the processing strategy with positive linear correlation was helpful to maintain the population diversity. Lin et al. [102] introduced a jumping-out strategy named crown jewel defense (CJD). CJD was used to relocate the gbest position and reinitialized all particles' pbest position when the swarm was trapped in local optima. Taking the advantage of CJD strategy, the swarm could jump out of the local optimal region without being dragged back and the performance of PSO became more robust to the initialization. Experimental results on benchmark functions showed that the CJD-based PSOs were comparable to or better than the other representative state-of-the-art PSOs. Wang and Watada [103] studied a facility location model with fuzzy random parameters and its swarm intelligence approach. A Value-at-Risk (VaR) based fuzzy random facility location model (VaR-FRFLM) was built in which both the costs and demands were assumed to be fuzzy random variables, and the capacity of each facility was unfixed but a decision variable assuming continuous values. A hybrid modified PSO approach was proposed to solve the VaR-FRFLM. Li et al. [104] developed a knowledge-based heuristic PSO approach with the adjustment strategy (KHPSOA), inspired by the No Free Lunch Theorem to solve the weighted circle packing problem. The numerical experiments showed that KHPSOA was superior to the existing algorithms in the performances. Inspired by the ecological behavior, Lu et al. [105] developed an augmented PSO (AugPSO) algorithm using two new 
strategies, boundary shifting and particle position resetting, with the aim of optimizing the design of truss structures. The boundary shifting approach forced particles to move to the boundary between feasible and infeasible regions in order to increase the convergence rate in searching. The particle position resetting approach was motivated by mutation scheme in GA to increase the diversity of particles and to prevent the solution of particles from falling into local minima. Mattos et al. [106] presented a constrained hybrid PSO algorithm in order to find feasible solutions to the resource allocation problem. Wu et al. [107] integrated some problem-oriented knowledge into the design of a certain PSO variant. They investigated the inner variable learning (IVL) strategy which could help the particle to inspect the relation among its inner variables, determine the exemplar variable for all other variables, and then make each variable learn from the exemplar variable in terms of their quantitative relations. Hence, a novel PSO algorithm with an IVL strategy was proposed and found particularly efficient for optimizing functions with symmetric variables. In addition, they designed a new trap detection and jumping-out strategy to help particles escape from local optima. Lim and Mat Isa [108] presented an adaptive two-layer PSO algorithm with elitist learning strategy (ATLPSO-ELS), which had better search capability than classical PSO. In ATLPSO-ELS, they performed evolution on both the current swarm and the memory swarm, motivated by the tendency of the latter swarm to distribute around the problem's optima. They proposed two adaptive divisionof-labor modules to self-adaptively divide the swarms into exploration and exploitation sections. An elitist learning strategy module was introduced in the proposed algorithm to enhance the search efficiency of swarms and to mitigate premature convergence. Shimizu et al. [109] developed a novel algorithm of PSO associated with binary decision variables. It was quite effective for finding the optimum opening distribution centers in three-echelon logistic network by parallel computing. Eventually, they had implemented the procedure in the parallel algorithm deployed as a multipopulation based approach using multithread programming technique. Fister Jr. et al. [110] presented a simple PSO, which allowed automatic creation of complex two-dimensional graphic characters. The method involved constructing the base characters, optimizing the modifications of the base characters with the PSO algorithm, and finally generating the graphic characters from the solution. They demonstrated the effectiveness of the approach with the creation of simple snowman.

3.2. Hybridization. PSO was combined with some traditional and evolutionary optimization algorithms in order to take the advantages of both methods and compensate the weaknesses of each other. This type of PSO is called hybridized PSO.

3.2.1. With GA. Kuo and Hong [111] presented a two-stage method of investment portfolio based on soft computing techniques. The first stage used data envelopment analysis to select most profitable funds, while hybrid of GA and PSO was proposed to conduct asset allocation in the second stage. Chen and Kurniawan [112] presented a two-stage optimization system to find optimal process parameters of multiple quality characteristics in plastic injection molding. Taguchi method, BPNN, GA, and combination of PSO and GA (PSO-GA) were used in this study to find optimum parameter settings. Nazir et al. [113] extracted facial local features using local binary pattern (LBP) and then fused these features with clothing features, which enhanced the classification accuracy rate remarkably. In the following step, PSO and GA were combined to select the most important features' set that more clearly represented the gender and thus the data size dimension was reduced. Vidhya and Kumar [114] proposed a hybrid technique that included PSO and GA for channel estimation in MIMO-orthogonal frequency division multiplexing (MIMO-OFDM) systems. The result showed the performance of the proposed method was better than LS and MMSE methods in all the mutation and crossover values and in all the iterations computed. Xiao et al. [115] constructed three different types of neural network based models, that is, Elman network, generalized regression neural network (GRNN), and wavelet neural network (WNN) constructed by three nonoverlapping training sets. Their empirical results suggested the ensemble ANNs-PSO-GA approach significantly improved the prediction performance over other individual models and linear combination models. Ghamisi and Benediktsson [116] proposed a new feature selection approach based on the integration of a GA and PSO. The overall accuracy of a support vector machine (SVM) classifier on validation samples was used as a fitness value. The new approach was carried out on the well-known Indian Pines hyperspectral dataset. Results confirmed that the new approach was able to select automatically the most informative features in terms of classification accuracy within an acceptable processing time without requiring the number of desired features to be set a priori by users.

3.2.2. With AIS. Tang et al. [117] presented a novel dynamic PSO algorithm based on improved artificial immune network (IAINPSO). Based on the variance of the population's fitness, a kind of convergence factor was adopted in order to adjust the ability of search. The experimental results showed that not only did the new algorithm satisfy convergence precision, but also the number of iterations was much less than traditional scheme and had much faster convergent speed, with excellent performance in the search of optimal solution to multidimensional function. Zhang et al. [118] proposed a more pragmatic model for stochastic networks, which considered not only determinist variables but also the mean and variances of random variables. In order to accelerate the solution of the model, they integrated PSO with chaos operator and AIS. Ibrahim et al. [119] developed a power quality monitor positioning algorithm to find the optimal number and placement of PQMs in both transmission and distribution systems. First, the concept of topological monitor reach area was introduced. Then the binary PSO hybridized with AIS was used to solve multiobjective function in finding the optimal placement of PQMs. Kuo et al. [120] intended to propose a hybrid of AIS and PSO-based SVM (HIP-SVM) for optimizing SVM parameters and applied it to radio 
frequency identification (RFID) based positioning system. The computational results showed that HIP-SVM had better performance than AIS-based SVM and PSO-based SVM. Liu et al. [121] proposed a coevolutionary PSO algorithm associating with the artificial immune principle. In the proposed algorithm, the whole population was divided into two kinds of subpopulations consisting of one elite subpopulation and several normal subpopulations. The best individual of each normal subpopulation will be memorized into the elite subpopulation during the evolution process. Darzi et al. [122] incorporated PSO, dynamic mutated AIS (DM-AIS), and gravitational search algorithm (GSA) into the existing LCMV technique in order to improve the weights of LCMV. The simulation result demonstrated that received signal to interference and noise ratio (SINR) of target user can be significantly improved by the integration of PSO, DM-AIS, and GSA in LCMV through the suppression of interference in undesired direction.

3.2.3. With TS. Li et al. [123] integrated nonlinear simplex method (NSM) into PSO in order to increase its convergence speed. They also integrated TS into PSO to assign Tabu attribute to local solution regions. The hybrid PSO algorithm was an organic composition of the PSO, NSM, and TS algorithms. Nakano et al. [124] presented a new form of PSO based on the concept of TS. The proposed Tabu list PSO (TLPSO) was a method for combining the strong points of PSO and TS. This method stored the history of pbest in a Tabu list. When a particle had a reduced searching ability, it selected a pbest of the past from the historical values, which was used for the update. This made each particle active, and the searching ability of the swarm made progress. Zhang et al. [125] presented the production planning management architecture for iron-steel manufacturing factories based on make-toorder and make-to-stock management ideas. In order to solve this nonlinear integer program, the authors designed a hybrid PSO and TS algorithm, in which new heuristic rules were proposed to repair infeasible solutions. Ktari and Chabchoub [126] proposed a new heuristic approach such that various features inspired from the TS were incorporated in the Essential Particle Swarm Optimization queen (EPSOq) algorithm in order to obtain another improved discrete PSO version. Wang et al. [127] focused on long-term distribution system maintenance scheduling aided by available operation information. A combined algorithm that consisted of PSO and TS was designed and applied to the optimization problem. Numerical result verified that the proposed method could schedule long-term maintenance of distribution systems in smart grid economically and effectively.

3.2.4. With ACO. Chen and Chien [128] presented a new method, called the genetic simulated annealing ant colony system with particle swarm optimization techniques, for solving the TSP. The experimental results showed that both the average solution and the percentage deviation of the average solution to the best known solution of the proposed method were better than existing methods. Xiao et al. [129] considered the features of the MRCMPSP problem. They employed ant colony's labor division to establish a task priority-scheduling model firstly. Then, they used improved PSO to find out the optimum scheduling scheme. The approach integrating the above two algorithms had abilities of both local search and global search. Kiran et al. [130] proposed a new hybrid method for estimating energy demand of Turkey using PSO and ACO. PSO was developed for solving continuous optimization problems; ACO was used for discrete optimizations. Hybrid method based PSO and ACO was developed to estimate energy demand using gross domestic product, population, import, and export. Huang et al. [131] incorporated ACOR with PSO to improve the search ability, investigating four types of hybridization as follows: (1) sequence approach, (2) parallel approach, (3) sequence approach with an enlarged pheromone-particle table, and (4) global best exchange. Among the four strategies of hybridization, the sequence approach with the enlarged pheromone table was superior to the other approaches because the enlarged pheromone table diversified the generation of new solutions of ACOR and PSO, which prevented traps into the local optimum. Rahmani et al. [132] used a new hybrid swarm technique (HAP) to forecast the energy output of a real wind farm located in Binaloud, Iran. The technique consisted of the hybridization of the ACO and PSO, which were two metaheuristic techniques under the category of swarm intelligence. The hybridization of the two algorithms to optimize the forecasting model led to a higher quality result with a faster convergence profile. Elloumi et al. [133] illustrated a novel optimization approach based on multiobjective PSO (MOPSO) and Fuzzy ACO (FACO). The basic idea was to combine these two techniques using the best particle of the Fuzzy Ant algorithm and integrate it as the best local PSO to formulate a new approach called hybrid MOPSO with FACO (H-MOPSO-FACO). This hybridization solved the multiobjective problem, which relied on both time performance criteria and the shortest path.

3.2.5. With SA. Sait et al. [134] proposed a hybrid of PSO and SA for solving the cell assignment in Cnnos\nanowire $\backslash$ MOLecular Hybrid (CMOL). Results showed that the proposed hybrid algorithm achieved better solution in terms of buffer count in reasonable time. Jiang and Zou [135] proposed an improved parameter optimization method based on traditional PSO algorithm by changing the fitness function in the traditional evolution process of SVMs. Then, this PSO method was combined with SA global searching algorithm to avoid local convergence that traditional PSO algorithms usually run into. This method achieved better results that reflected in the ROC curves in medical images classification and gained considerable identification accuracy in clinical disease detection. Niknam et al. [136] proposed a hybrid PSO and SA (PSO-SA) method to solve the dynamic optimal power flow (DOPF) problem while the prohibited zones, valve-point effects, and ramp rate constraints were taken into account. The hybrid PSO-SA algorithm could do an efficient search and explore solution space, while it profited from privileges of both PSO and SA algorithms. Khoshahval et al. [137] developed a new parallel optimization algorithm, P-PSOSA, 
for performing the fuel management optimization; they defined two different fitness function considering the multiplication factor maximizing and power peaking factor minimizing objectives simultaneously. Numerical results of P-PSOSA confirmed that the proposed algorithm had a great strength to obtain a near global core pattern with respect to considered objective functions during suitable consuming runtime. Du et al. [138] presented a hybrid algorithm based on improved PSO and SA (IPSOSA) algorithm to solve the resource constrained multiproject scheduling problem. Aimed at overcoming the shortcomings of premature convergence of standard PSO, adaptive inertia weight with cyclical attenuation strategy and SA were employed in the hybrid algorithm. Zhang et al. [139] proposed an improved approach to decompose structuring elements of an arbitrary shape. They introduced in the restarted simulated annealing PSO method, which was the combination of restarted SA and PSO. Geng et al. [140] introduced robust v-support vector regression (RSVR) model to forecast port throughput. In order to search the more appropriate parameters combination for the RSVR model, they presented a chaotic simulated annealing PSO (CSAPSO) algorithm to determine the parameter combination.

3.2.6. With ABC. El-Abd [141] tested a hybrid PSO and ABC algorithm on the CEC13 testbed. The hybridization technique was a component-based one, where the PSO algorithm was augmented with an $\mathrm{ABC}$ component to improve the personal best of the particles. Sharma et al. [142] proposed a variant called Local Global variant ABC (LGABC) to balance the exploration and exploitation in $\mathrm{ABC}$. The proposal harnessed the local and global variant of PSO into ABC. The proposed variant was investigated on a set of thirteen well-known constrained benchmarks problems and three chemical engineering problems, which showed that the variant can get high-quality solutions efficiently. Kiran and Gündüz [143] presented a hybridization of PSO and ABC approaches, based on recombination procedure. The global best solutions obtained by the PSO and ABC were used for recombination, and the solution obtained from this recombination was given to the populations of the PSO and ABC as the global best and neighbor food source for onlooker bees, respectively. Information flow, between particle swarm and bee colony, helped increase global and local search abilities of the hybrid approach. Vitorino et al. [144] put forward a mechanism based on the $\mathrm{ABC}$ to generate diversity when all particles of the PSO converged to a single point of the search space. Then, the swarm entities switched between two predefined behaviors by using fuzzy rules depending on the diversity of the whole swarm.

3.2.7. With DE. Maione and Punzi [145] proposed a two-step design approach. First, DE determined the fractional integral and derivative actions satisfying the required time-domain performance specifications. Second, PSO determined rational approximations of the irrational fractional operators as low-order, stable, minimum-phase transfer functions with poles interlacing zeros. Extensive time- and frequencydomain simulations validated the efficiency of the proposed approach. Fu et al. [146] presented a hybrid DE with QPSO for the unmanned aerial vehicle (UAV) route planning on the sea. It combined DE algorithm with the QPSO algorithm in an attempt to enhance the performance of both algorithms. Experimental results demonstrated that the proposed method was capable of generating higher quality paths efficiently for UAV than any other tested optimization algorithms. Vasundhara et al. [147] presented an efficient way of designing linear-phase finite impulse response (FIR) lowpass and high-pass filters using a novel algorithm ADEPSO, which was hybrid of fitness based adaptive DE and PSO. ADEPSO overcame the above individual disadvantages faced by both algorithms and was used for the design of linearphase low-pass and high-pass FIR filters. The simulation results showed that the ADEPSO outperformed PSO, ADE, and DE in combination with PSO not only in magnitude response but also in the convergence speed and thus proved itself to be a promising candidate for designing the FIR filters. Yu et al. [148] formulated a novel adaptive hybrid algorithm based on PSO and DE (HPSO-DE) by developing a balanced parameter between PSO and DE. Adaptive mutation was carried out on current population when the population clustered around local optima. The HPSO-DE enjoyed the advantages of PSO and DE and maintained diversity of the population. Compared with PSO, DE, and their variants, the performance of HPSO-DE was competitive. Wang et al. [149] proposed a robust hybrid metaheuristic optimization approach by adding DE mutation operator to the accelerated PSO (APSO) algorithm to solve numerical optimization problems. Yadav and Deep [150] proposed a new co-swarm PSO (CSHPSO) for constrained optimization problems, which was obtained by hybridizing shrinking hypersphere PSO (SHPSO) with the DE approach. The total swarm was subdivided into two subswarms in such a way that the first subswarms used SHPSO and second subswarms used DE. Experiments showed that CSHPSO was a promising new coswarm PSO which could be used to solve any real constrained optimization problem.

3.2.8. With Other Approaches. Xu et al. [151] proposed a hybrid PSO integrated with trust region method. The simulation results on some multimodal global optimizations showed that the algorithm was far more effective than linear decreasing weight PSO (LDWPSO) and sequential quadratic programming (SQP) to search the global optimum. Mohanty et al. [152] presented a study on frequency regulation in isolated hybrid DG system. H-infinite loop shaping based on PSO as well as hybrid PSO and HS (PSOHS) controller was proposed to minimize the frequency deviation. Guo et al. [153] employed PSO and BBO to propose a hybrid algorithm termed biogeography-based PSO (BPSO), which could make a large number of elites effective in searching optima. The whole population was split into several subgroups; BBO was employed to search within each subgroup and PSO for the global search.

3.3. Extensions. The success of the PSO algorithm as a single-objective optimizer within continuous search space 
has motivated researchers to extend its use to other areas, which consist of but not limited to multiobjective optimization, constrained optimization, binary optimization, discrete optimization, combinatorial optimization, and so forth.

\subsubsection{Multiobjective Optimization. Multiple objective PSO} (MOPSO) has been proposed to deal with multiobjective optimization problems, in which the objective function took Pareto dominance into account when moving the PSO particles and nondominated solutions were stored so as to approximate the Pareto front. Qiu et al. [154] proposed a MOPSO with a new gbest selection strategy. They used $K$-means algorithm and proportional distribution based approach to select gbest from the archive for each particle of the population. A symmetric mutation operator was incorporated to enhance the exploratory capabilities. The simulation results indicated that the proposed algorithm was highly competitive in terms of convergence and diversity in comparison with several state-of-the-art algorithms. Chen et al. [155] presented an ironless permanent magnet linear brushless motor with three objective functions: maximal thrust force, minimal temperature, and minimal volume. An elitist hybrid QPSO algorithm with mutation was used to solve this multiobjective optimization problem. Elitist mechanism with crowding distance sorting was used to improve the number and diversity of the solutions. Ghanei et al. [156] presented application of thermal-economic multiobjective optimization of shell and tube heat exchanger (STHE) using MOPSO to obtain the maximum effectiveness (heat recovery) and the minimum total cost as two objective functions. Duan et al. [157] developed a mathematical model based on thermodynamic analysis of Stirling engine considering regenerative losses and internal irreversibilities. Power output, thermal efficiency, and the cycle irreversibility parameter of Stirling engine were optimized simultaneously using MOPSO. Amiryousefi et al. [158] performed a multiobjective optimization for deep-fat frying of ostrich meat plates. MOPSO was used to obtain the best solutions. This problem had three objective functions that must be satisfied simultaneously. Results showed a Pareto where all the points on this Pareto were the best possible solutions. Ganguly [159] presented a PSObased multiobjective planning algorithm for reactive power compensation of radial distribution networks with unified power quality conditioner (UPQC) allocation. The optimal location, the optimal reactive power compensation required at the location, and the optimal design parameters of UPQC were determined by minimizing three objective functions: the rating of UPQC, network power loss, and percentage of nodes with undervoltage problem. These objectives were simultaneously minimized to obtain a set of nondominated solutions using MOPSO. Zhang et al. [160] combined BBPSO and sensitivity-based clustering for solving multiobjective reliability redundancy allocation problems (RAPs). A twostage process was performed to identify promising solutions. A new bare-bones MOPSO (BBMOPSO) was developed and applied in the first stage to identify a Pareto-optimal set. This algorithm mainly differed from other MOPSO algorithms in the parameter-free particle updating strategy, which was especially suitable for handling the complexity and nonlinearity of RAPs. Perera et al. [161] applied MOPSO to identify intermediate debonding damage in the problem of fiber-reinforced-polymer-composites- (FRP-) plated RC beams. The use of permanently installed fiber Bragg grating sensors embedded into the FRP concrete interface or bonded onto the FRP strip together with the proposed methodology resulted in an automated method able to operate in an unsupervised mode. Cheng et al. [162] proposed an improved MOPSO with preference strategy (IMPSO-PS) and applied it to the optimal integration of distributed generation (DG) into the distribution system. Preference factors were introduced to quantify the degree of preference for certain attributes in the constraint-space. In addition to the application of a popular nondominated sorting technique for identifying Pareto solutions, the performance of IMPSO-PS was strengthened via the inclusion of a dynamic selection of the global best, a novel circular nondominated selection of particles, and a special mutation operation.

\subsubsection{Constrained Optimization. Scholars have proposed} several solutions to constrained optimization problem. For example, Daneshyari and Yen [163] proposed a culturalbased constrained PSO to incorporate the information of the objective function and constraint violation into four sections of the belief space, specifically normative knowledge, spatial knowledge, situational knowledge, and temporal knowledge. The archived information facilitated communication among swarms in the population space and assisted in selecting the leading particles in three different levels: personal, swarm, and global levels. Afshar [164] presented three constrained versions of PSO algorithm for the efficient optimal operation of multireservoir systems using storage/release volumes as decision variables of the problem. Proposed algorithms were based on identifying and excluding the infeasible region of the search space before and during the search. Koulinas et al. [165] proposed a PSO-based hyperheuristic algorithm for solving the resource constrained project scheduling problem. The hyperheuristic worked as an upper-level algorithm that controlled several low-level heuristics which operated to the solution space. The solution representation was based on random keys. Active schedules were constructed by the serial scheduling generation scheme using the priorities of the activities which were modified by the low-level heuristics of the algorithm. Shan and Ren [166] combined PSO with direct approach and applied the method to low-thrust trajectory optimization problems. A double-loop trajectory optimization algorithm was developed. The outer loop of this algorithm was a modified PSO optimizer, which dealt with constrained optimization problems and avoided premature convergence. The direct approach (fourth-order Runge-Kutta shooting/parallel shooting method) was adopted as the inner loop algorithm, whose main task was to correct the particles provided by the outer loop and ensure that all the constraints were satisfied. Yeh and Chien [167] proposed an efficient algorithm for solving a class of constrained minimal spanning tree (MST) problems. The proposed PSO-like strategy for solving constrained MST problems identified optimal MSTs under 
degree and delay constraints. Singh et al. [168] presented a novel approach to detect a salient object which involved two phases. In the first phase, three features such as multiscale contrast, center-surround histogram, and color spatial distribution were obtained. Constrained PSO was used in the second phase to determine an optimal weight vector to combine these features to obtain saliency map to distinguish a salient object from the image background. Paliwal et al. [169] presented a systematic approach for determination of optimal mix of resources. The considered constituent resources comprised of diesel, photovoltaic (PV), wind, and battery storage. A technosocioeconomic criterion was formulated in order to determine optimum combination of resources. PSO was used to determine optimal component sizing for each of the configuration. Cui et al. [170] developed a multitarget PSO (mPSO) to solve the parallel model of independentcomponent-analysis constrained by a 5-parameter Reference Curve. Shou et al. [171] proposed a hybrid PSO procedure to solve the preemptive resource constrained project scheduling problem in which a maximum of one interruption per activity was allowed. Four types of particle representations were designed and two schedule generation schemes were adopted to decode the particle representations. Particleupdating mechanisms based on the peak crossover operator were designed for all particle representations.

3.3.3. Discrete Optimization. Discrete PSO (DPSO) was proposed and harnessed to address discrete optimization/integer programming problems. Chen and Ludwig [172] devised a PSO-based discrete implementation with a local search strategy (DPSO-LS). The local search strategy helped to overcome local optima in order to improve the solution quality. The DPSO-LS used the Pittsburgh approach whereby a rule base was used to represent a particle. This rule base evolved over time as to finding the best possible classification model. Shen et al. [173] proposed a novel bivelocity DPSO (BVDPSO) approach and extended its application to the nondeterministic polynomial complete multicast routing problem (MRP). First, a novel bivelocity strategy was developed to represent the possibilities of each dimension being 1 and 0 . Second, BVDPSO updated the velocity and position according to the learning mechanism of the original PSO in the continuous domain. Experiments showed BVDPSO outperformed not only several state-of-the-art and recent heuristic algorithms for the MRP problems, but also algorithms based on GA, ACO, and PSO. Chen et al. [174] proposed a revised DPSO (RDPSO) to solve the permutation flow-shop scheduling problem with the objective of minimizing makespan (PFSPmakespan). RDPSO proposed new particle swarm learning strategies to thoroughly study how to properly apply the gbest solution and the pbest solution to guide the search of RDPSO. A new filtered local search was developed to filter the solution regions that had been reviewed and guided the search to new solution regions in order to keep the search from premature convergence. Cai et al. [175] suggested using a novel DPSO for identifying community structures in signed networks. Particles' status had been redesigned in discrete form so as to make PSO proper for discrete scenarios. Particles' updating rules had been reformulated by making use of the topology of the signed network. Kashan et al. [176] presented a novel DPSO, which used group-based operators, in place of arithmetic operators, in the body of the updating equations analogous to those of the classical PSO equations. All operators in the new algorithm worked with constructed cells (groups) rather than parts/machines (objects). Xu et al. [177] employed a DPSO approach to solve the requirements contradiction between high transparency in pass band and high reflectance in stop band. Garg and Singh [178] used $\varepsilon$-fuzzy dominance sort based DPSO (FDPSO) approach to solve the workflow scheduling problem in the grid. The metric, fuzzy dominance which quantified the relative fitness of solutions in multiobjective domain was used to generate the Pareto optimal solutions. In addition, the scheme also incorporated a fuzzy-based mechanism to determine the best compromised solution. Zong et al. [179] proposed a DPSO with neighborhood learning factor algorithm to solve the temporal-spatial conflict and congestion for pedestrian-vehicle mixed evacuation. The proposed algorithm introduced a neighborhood learning factor to simulate the subgroup phenomenon among evacuees and to accelerate the evacuation process. Ezzeldin et al. [180] used integer DPSO as an optimization technique for the design of water distribution networks in order to minimize its total cost. Because the particle swarm was highly sensitive to its parameters and boundary conditions, the available restricted boundary conditions were applied. Also, a new boundary condition called the billiard boundary condition was introduced, which did not depend on the velocity clamping that mainly depended on human assumptions.

\subsubsection{Binary Integer Programing. 0-1 integer programming} or binary integer programming (BIP) is the special case of integer programming where variables are required to be 0 or 1. Binary PSO (BPSO) was used to solve this type of problems. Zhai and He [181] proposed a new immune BPSO (IBPSO) to solve the problem of instance selection for time series classification, whose objective was to find out the smallest instance combination with maximal classification accuracy. The proposed IBPSO was based on the BPSO algorithm. Its immune mechanism included vaccination and immune selection. Sarath and Ravi [182] developed a BPSO based association rule miner, which generated the association rules from the transactional database by formulating a combinatorial global optimization problem, without specifying the minimum support and minimum confidence unlike the a priori algorithm. The quality of the rule was measured by a fitness function defined as the product of support and confidence. Taha and Abu Al Nadi [183] presented a maximum likelihood estimate (MLE) to detect the number of vacant channels in the spectrum. The resulting MLE needed exhaustive search to be determined accurately. BPSO was proposed to solve the problem. Simulation results had shown significant improvement of the MLE-BPSO estimated over the conventional techniques by 3-5 dB. El-Maleh et al. [184] proposed an improved BPSO algorithm and demonstrated its effectiveness in solving the state assignment problem 
in sequential circuit synthesis targeting area optimization. Experimental results demonstrated the effectiveness of the proposed BPSO algorithm in comparison to other BPSO variants and in comparison to GA, SimE, and deterministic algorithms like Jedi and Nova. Erturk et al. [185] proposed a novel spatial resolution enhancement method using fully constrained least squares (FCLS) spectral unmixing and spatial regularization based on modified BPSO to achieve spatial resolution enhancement in hyperspectral images, without using an additional image with higher spatial resolution. The proposed method had a highly parallel nature with respect to its counterparts in the literature and was fit to be adapted to field-programmable gate array architecture. Zhang et al. [186] proposed a novel spam detection method that focused on reducing the false positive error of mislabeling nonspam as spam. BPSO with mutation operator (MBPSO) was employed as the subset search strategy. The results showed that the MBPSO performed better than sequential forward selection (SFS) and sequential backward selection (SBS). Yin et al. [187] used a feature selection algorithm according to the separability criterion to preselect the discrete cosine transform (DCT) coefficients and then employed search algorithm based on BPSO and SVM to find an optimal combination of the DCT coefficients. Yang et al. [188] proposed a modified version of BPSO (MBPSO), which adopted a different transfer function and a new position updating procedure with mutation, for the task allocation problem to obtain the best solution. Each particle in MBPSO was encoded to represent a complete potential solution for task allocation. The task workload and connectivity were ensured by taking them as constraints for the problem. Multiple metrics, including task execution time, energy consumption, and network lifetime, were considered a whole by designing a hybrid fitness function to achieve the best overall performance. Ganesh et al. [189] proposed entropic BPSO (EBPSO), which generated an entropy map, the highest value of which was used to localize the ear in a face image. Experimental results showed the promising performance of EBPSO for ear detection on four benchmark face databases: CMU PIE, Pointing Head Pose, Color FERET, and UMIST.

\subsection{Theoretical Analysis}

3.4.1. Parameter Choice. The choice of parameters of PSO has a large impact on its optimization performance [190]. Therefore, how to select or tune the parameters yielding good results had been the hot topic. Kumar and Chaturvedi [191] developed a FPSO, in which inertia weight was adaptively adjusted using fuzzy logic controller (FLC) during the search process. The FLC presented had one input and one output into PSO. Zhang et al. [192] proposed a simple way to estimate the nonnegative real parameter tuple (omega, $c_{1}$, and $c_{2}$ ) of standard PSO algorithm using control theory. The distribution of complex characteristic roots on the convergence region of particles was studied by means of linear discrete-time system analysis method. Yang [193] proposed an improved PSO variant, called PSO with modified velocity strategy (MVPSO), in which each particle was attracted by the global best particle and a random particle chosen from a set of good particles. Simulation results showed that MVPSO obtained better performance than standard PSO and two other improved PSO variants. Sun et al. [194] presented a comprehensive analysis of the QPSO algorithm. They analyzed the behavior of a single particle in QPSO in terms of probability measure. Since the particle's behavior was influenced by the contraction-expansion (CE) coefficient, the goal of the theoretical analysis was to find out the upper bound of the CE coefficient, within which the value of the CE coefficient selected could guarantee the convergence or boundedness of the particle's position. Yassin et al. [195] found that BPSO algorithm was subject to several parameters: swarm size, maximum iterations, and initial positions. They investigated the effect of the swarm size parameter on the convergence of BPSO. The results over DC motor dataset indicated that the optimal swarm size for convergence was between 20 and 30 particles. Wang et al. [196] compared and analyzed the optimization performance of PSO under different parameters, in order to guarantee the convergence of PSO applied to the inverting of ellipsometry. The result showed that the range of inertia weight omega from 0.5 to 0.8 , the sum of learning parameters $c_{1}$ and $c_{2}$ preferably no more than 3 , and a smaller $c_{1}$ and a bigger $c_{2}$ ensured the better optimization performance of PSO. Hao et al. [197] proposed molecular force model based PSO (MFMPSO). Two parameters were introduced in the MFMPSO algorithm. The orthogonal test design method was applied to optimize the parameter combinations of three levels and four factors, which included $d(1)$ and $d(h)$, the population size, and the iteration number. Xu [198] proposed an adaptive parameter tuning of PSO based on velocity information (APSO-VI). This algorithm introduced the velocity information defined as the average absolute value of velocity of all the particles. A new strategy presented that the inertia weight was dynamically adjusted according to average absolute value of velocity, which followed a given nonlinear ideal velocity by feedback control. Under the guide of the nonlinear ideal velocity, APSO-VI maintained appropriate swarm diversity and alleviated the premature convergence validly. Chauhan et al. [199] proposed three new nonlinear strategies for selecting inertia weight which played a significant role in particle's foraging behavior. The PSO variants implying these strategies were named fine grained inertia weight PSO (FGIWPSO), double exponential self-adaptive IWPSO (DESIWPSO), and double exponential dynamic IWPSO (DEDIWPSO). In FGIWPSO, inertia weight was obtained adaptively, depending on particle's iteration wise performance, and decreased exponentially. DESIWPSO and DEDIWPSO employed Gompertz function, a double exponential function for selecting inertia weight. In DESIWPSO the particles' iteration wise performance was fed as input to the Gompertz function. On the other hand, DEDIWPSO evaluated the inertia weight for whole swarm iteratively using Gompertz function where relative iteration was fed as input. Zhang et al. [200] found that engineering experience could be used to determine the parameters of an optimization algorithm. They analyzed the dynamic characteristics of PSO through a large number of experiments and constructed a relationship between 
the dynamic process of PSO and the transition process of a control system. A novel parameter strategy for PSO was proven using the overshoot and the peak time of a transition process. This strategy provided not only a series of flexible parameters for PSO but also a new way to analyze particle trajectories that incorporated engineering practices. The experimental results showed that the proposed strategy was effective and easy to implement. Kanemasa and Aiyoshi [201] combined a feedback element as an algorithm tuner with an original algorithm; the resulting algorithm was applied to the optimization problem in question. They used genetic programming (GP) to generate tuning rules to automatically tune the PSO algorithm, namely, augmented PSO by using GP as a meta-algorithm to solve the learning problem of automatically generating tuning rules for the parameters in the PSO algorithm.

3.4.2. Convergence Analysis. PSO may converge to global optimal or local optimal positions. The latter is unexpected for PSO-users. Attempts at mathematically analyzing PSO convergence exist in literatures. These analyses offer guides for selection parameters of PSO in another way that guarantees global convergence and avoid premature. Wang and Shen [202] gave the general mathematical description of PSO. Afterwards, they proved that the solution space of PSO in normed space and the iterative relations of PSO were contraction mapping. Using the theorem of Banach space and contraction mapping principle, they proved the existence and uniqueness of the convergence position. Sun et al. [203] presented a convergence analysis and performance evaluation of QPSO algorithm. They investigated in detail the convergence of the QPSO algorithm on a probabilistic metric space and proved that the QPSO algorithm was a form of contraction mapping and converged to the global optimum. It was the first time that the theory of probabilistic metric spaces had been employed to analyze a stochastic optimization algorithm. They provided a new definition for the convergence rate of a stochastic algorithm as well as definitions for three types of convergence according to the correlations between the convergence rate and the objective function values. Kurihara and Jin'no [204] analyzed the convergence property of the PSO and its application to the nonlinear blind source separation system. The interparticle communication of the PSO was realized by the past history of the neighbors and depended on the network structure of the swarm. Lin [205] considered the router node placement of wireless mesh networks in a dynamic network scenario. They first modelled a mathematical form for the concerned problem, then proposed a PSO approach, and, from a theoretical aspect, provided the convergence and stability analysis of the PSO with constriction coefficient, which was much simpler than the previous analysis. Zhang et al. [206] studied an improved BBPSO algorithm with adaptive disturbance (ABPSO). They used stochastic process theory to analyze the convergence of ABPSO, by regarding each particle's position as a stochastic vector. Lin et al. [207] thought the PSO had a tendency to get stuck in a near-optimal solution especially for middle and large size problems, and it was difficult to improve solution accuracy by fine-tuning parameters. Hence, they proposed a local and global search combined PSO (LGSCPSOA), analyzed its convergence, and obtained its convergence qualification. Kim and $\mathrm{Li}$ [208] suggested the conventional derivative-based estimation approach was often terminated earlier without converging due to the singularity if a model was statistically nonidentifiable. To circumvent this difficulty, a derivative-free global optimization algorithm was developed by combining PSO with a derivative-free local optimization algorithm to improve the rate of convergence of PSO. They further checked the convergence of the proposed method.

3.5. Parallel Implementation. Parallel computing is a computational form, in which computations are carried out simultaneously. On one hand, computers using multicore, multiprocessor, and graphics processing unit (GPU) contain multiple processing elements within a single machine. On the other hand, clusters, grids, and clouds employed multiple computers to work on the same task.

3.5.1. Multicore. PSO can be implemented on multicore (multiprocessor) conditions. Waintraub et al. [209] used parallel computation to overcome the huge computational costs required by PSO. They investigated the master-slave approaches and developed several different PPSO algorithms exploring the advantages of enhanced neighborhood topologies implemented by communication strategies in multiprocessor architectures. Yu [210] proposed the incorporation of a local search heuristic into the basic PSO algorithm. The new, hybrid metaheuristic was called twin PSO (TPSO). The proposed metaheuristic scheme was applied to a flow shop with multiprocessors scheduling problem.

3.5.2. GPU Computing. GPU is a specialized electronic circuit designed to rapidly manipulate and alter memory to accelerate the creation of images in a frame buffer intended for output to a display. Modern GPUs are very efficient at manipulating computation of PSO. Hung and Wang [211] focused on the acceleration of PSO for solving box-constrained, load-balanced optimization problems by parallelization on a GPU. They proposed a GPU-accelerated PSO (GPSO) algorithm by using a thread pool model and implement GPSO on a GPU. Numerical results showed that the GPU architecture fitted the PSO framework well by reducing computational timing, achieving high parallel efficiency, and finding better optimal solutions by using a large number of particles. Rymut et al. [212] discussed how to combine particle filter (PF) with PSO to achieve better object tracking. They also proposed a parallel resampling scheme for particle filtering running on GPU. They showed the efficiency of the parallel PF-PSO algorithm on 3D model based human motion tracking. Kumar et al. [213] thought the applications requiring massive computations got benefit from the GPU with compute unified device architecture (CUDA) by reducing the execution time. Hence, they presented a detailed study of parallel implementation of the cooperative PSO (CPSO). They also presented a comparative study on 
CPSO implemented in C and C-CUDA. Awwad et al. [214] applied GPU computing solution based on CUDA to solve the topology control problem in hybrid radio frequency and free space optics wireless mesh networks by adapting and adjusting the transmission power and the beam-width of individual nodes according to QoS requirements. Their approach was based on a PSO and was implemented on the NVIDIA GeForce GTX 285 GPU. The implementation achieved a performance speedup factor of 392 over a CPUonly implementation. Chen et al. [215] proposed a PSO-based algorithm to efficiently find optimal uniform designs with respect to the central composite discrepancy (CCD) criterion. Parallel computation techniques based on state-of-the-art GPU were employed to accelerate the computations. They further demonstrated that the proposed algorithm could be extended to incorporate desirable space-filling properties, such as the noncollapsing property.

3.5.3. Cloud Computing. Cloud computing is a computing form in which large groups of remote servers are networked to allow centralized data storage and online access to computer services or resources. Scholars studied the performance of PSO implemented in cloud computing. Liu et al. [216] established a job scheduling model based on the PSO algorithm for cloud computing to reduce the energy consumption and improve the profit. Based on open source cloud computing simulation platform CloudSim, compared to GA and random scheduling algorithms, the results showed that the proposed algorithm obtained a better solution concerning the energy cost and profit. Xu and You [217] minimized the thermal residual stresses (TRS) of the unidirectional ceramic matrix composites (CMC) with multilayered interphases by controlling the interphases thicknesses. The MapReduce was extended to a new iterative MapReduce, which was combined with classical PSO algorithm to develop an iterative MapReduce guided PSO (IMPSO) algorithm. The IMPSO algorithm was interfaced with finite element code to find an optimal design for minimizing the TRS within CMCs. Ramezani et al. [218] developed a comprehensive multiobjective model for optimizing task scheduling to minimize task execution time, task transferring time, and task execution cost. They designed a multiobjective algorithm based on MOPSO method to provide an optimal solution for the proposed model. To implement and evaluate the proposed model, they extended Jswarm package to multiobjective Jswarm (MO-Jswarm) package. They also extended CloudSim toolkit applying MOJswarm as its task scheduling algorithm. Govindarajan et al. [219] captured the data from students and analyzed and clustered the data based on their individual performances in terms of accuracy, efficiency, and quality. The clustering process was carried out by employing PSO. The proposed PSO-based clustering was compared with existing $K$-means algorithm for analyzing the performance of intercluster and intracluster distances. Finally, the processed data was effectively stored in the Cloud resources using Hadoop distributed file system (HDFS). Ramezani et al. [220] proposed a taskbased system load balancing method using PSO (TBSLBPSO) that achieved system load balancing by only transferring extra tasks from an overloaded VM instead of migrating the entire overloaded VM. They designed an optimization model to migrate these extra tasks to the new host VMs by applying PSO. To evaluate, they extended the cloud simulator (CloudSim) package and used PSO as its task scheduling model.

\section{Applications of PSO}

PSO has been used in many applications in various academic and industrial fields so far. Using the analytical tool provided by "Web of Science Core Collection," the hottest application categories are "electrical and electronic engineering," "automation control systems," "communication theory," "operations research," "mechanical engineering," "fuel and energy," "medicine," "chemistry," "biology," and so forth.

4.1. Electrical and Electronic Engineering. PSO was used by researchers to solve the optimization problems encountered in electrical and electronic engineering. Ganguly et al. [221] presented a multiobjective planning approach for electrical distribution systems under uncertainty in load demand incorporating DG. The optimization tool was a MOPSO variant that used heuristic selection and assignment of leaders or guides for efficient identification of nondominated solutions. Komsiyah [222] used Gaussian PSO and Lagrange multiplier to solve the EDP of electric power generation, scheduling the committed generating units outputs so as to meet the required load demand at minimum operating cost, while satisfying all units and system equality and inequality constraint. Feng et al. [223] employed orthogonal signal correction and PSO in order to detect wound infection by and improve the performance of electronic nose. Pekşen et al. [224] proposed a PSO method for estimating the model parameters of a layered anisotropic earth model such as horizontal and vertical resistivities and thickness. The result was promising and the proposed method could be used for evaluating one-dimensional direct current data in anisotropic media. Yang et al. [225] proposed a novel electric vehicle (EV) charging model. A PSO algorithm was proposed for the model optimization. Simulation results showed that the proposed strategy could reduce the operational cost of the power grid considerately, while meeting the EV owner's driving requirement. de Mendonça [226] used PSO and heuristic information to find the minimum expansion cost of the electrical energy transmission system. The proposed methodology was applied to the Garver system and to two real equivalent systems for the south and southeast of Brazil. Liu et al. [227] employed DPSO to systematically investigate the structural stability and features of tetrahexahedral Ptbased bimetallic nanoparticles with high-index facets. Aich and Banerjee [228] applied SVM for developing the model of electrical discharge machining process, with the aim of predicting the output with reasonable accuracy. PSO was employed for the purpose of optimizing SVM parameter combinations. Chou et al. [229] surveyed defects of reservoir grounding system, considering the performance of lightning protection and improved design, based on soil drilling data and the PSO technique. They showed PSO was indeed 
better than conventional planning and design method. Lee et al. [230] applied an electrical resistance tomography technique combining the PSO algorithm with the Gauss-Newton method, to the visualization of two-phase flows. Thakral and Bakhshi [231] employed PSO with negative factor counting technique and inverse iteration method, for designing novel binary and ternary copolymers based on thiophene, pyrrole, and furan skeletons. Fister et al. [232] tried to use the semantic tools such as resource definition framework (RDF) and RDF query language (SPARQL) for the optimization purpose. These tools were combined with PSO and the selection of the best solutions depends on its fitness. Aghaei et al. [233] presented a multiobjective optimization algorithm for the multistage distribution expansion planning (MDEP) in the presence of DGs using nonlinear formulations. The objective functions of the MDEP consisted of minimization of costs, energy-not-distributed, active power losses, and voltage stability index based on short circuit capacity. A modified PSO algorithm was developed and used for this multiobjective MDEP optimization. Selakov et al. [234] proposed a practical new hybrid model for short-term electrical load forecasting based on PSO and SVM. Proposed PSO-SVM model was targeted for forecast load during periods with significant temperature variations. The proposed model detected periods when temperature significantly changed based on weather (temperature) forecast and decided whether the model could be trained just on recent history. Shirvany et al. [235] used a modified PSO to solve the electroencephalography (EEG) source localization problem. The method was applied to noninvasive EEG recording of somatosensory evoked potentials for a healthy subject. Tungadio et al. [236] discussed the application of PSO to solve the state estimation problem, that is, to estimate the state variables of the power system by minimizing all measurement errors available at the control center. PSO was shown to be more effective than GA.

4.2. Automatic Control System. PSO has found several applications in automatic control systems. Cai and Yang [237] proposed an improved PSO-based approach for a team of mobile robots to cooperatively search for targets in complex unknown environments. The improved cooperation rules for a multirobot system were applied in the potential field function, which acted as the fitness function of the PSO. Kolomvatsos and Hadjieftymiades [238] held a negotiation between intelligence agents, which undertook the responsibility of representing buyers and sellers and negotiated over the conclusion of purchases. They studied concurrent negotiations between a buyer and a set of sellers. The PSO algorithm was adopted by each thread, which followed a specific strategy and adopted swarm intelligence techniques for achieving the optimal agreement. Pandey et al. [239] presented the load frequency control (LFC) problem using different optimization algorithms for two types of power system configurations. The control scheme proposed was based on linear matrix inequalities with its parameters tuned by PSO. Śtimac et al. [240] applied PSO to tune PID controller, using active magnetic bearing that suspended the rotating shaft and maintained it in levitated position by applying controlled electromagnetic forces on the rotor in radial and axial directions. Nedic et al. [241] proposed a new cascade load force control design for a parallel robot platform. A parameter search based on PSO was suggested to effectively search the parameters of the cascade controller. Simulation results showed the advantages of the proposed optimal tuned cascade controller to solve the formulated tracking problem in relation to the classical proportionalintegral controller. Chang and Chen [242] aimed at the PID control system design for multivariable input and multivariable output (MIMO) processes. An improved version of PSO algorithm was utilized to design PID control gains in MIMO control systems. The velocity updating formula of the developed algorithm included a new factor, that is, the best particle of each subpopulation, to enhance the search capacity. Xiang et al. [243] proposed a new sensorless control scheme without the use of a position feedback sensor. With the help of the system identification technique and PSO, the control scheme showed a satisfactory command tracking performance for the conducting polymer actuator's step and dynamic displacement responses, especially under a disturbance, without needing a physical feedback loop, but using a simulated feedback loop. Danapalasingam [244] presented an autonomous helicopter stabilization in the presence of a wind disturbance. A controller was designed based on the nonlinear adaptive output regulation and robust stabilization of a chain of integrators by a saturated feedback to stabilize the position and velocity of a helicopter. PSO was applied to automate the tuning process. Mahmoodabadi et al. [245] presented a novel MOPSO called IngeniousMOPSO and compared its capability with three well-known multiobjective optimization algorithms. They applied the proposed method to an intellectual challenge in robotics, that is, a biped robot walking in the lateral plane on slope. Zhong et al. [246] presented an improved PID intelligent control algorithm, which was applied to the electric gas pressure regulator. The algorithm used the improved radial basis function (RBF) neural network based on PSO algorithm to make online adjustment on PID parameters. Both theoretical analysis and simulation result showed that the algorithm shortened the step response time and improved tracking performance. Perng et al. [247] proposed a combination of PSO and RBFNN to determine the optimal operating point for the PID controller of a wind turbine and to identify the stability regions in the parameter space. Huang and Li [248] utilized an improved PSO with bounded constraints technique on velocity and positioning for adjusting the gains of a PID and iterative learning control (MC) controllers. Simulations and experiment results showed that the proposed controller could reduce the error significantly after two learning iterations. The developed method using bounded constraints technique provided valuable programming tools to practicing engineers. Nisha and Pillai [249] demonstrated control accuracy and computational efficiency of nonlinear model predictive control (NMPC) strategy, which utilized a probabilistic sparse kernel learning technique called relevance vector regression (RVR) and PSO with controllable random exploration velocity (PSO-CREV). 
4.3. Communication Theory. An interesting application area of PSO is communication theory. Yousefi et al. [250] presented the design procedure and implementation of a dual band planar quadrature hybrid with enhanced bandwidth. Small dual frequency transformers in two sections were used to modify conventional broad band hybrid coupler for having arbitrary dual band operation frequencies. By merging compilers and full-wave simulators, PSO was used to achieve proper values to have desired goals. Sun et al. [251] applied PSO algorithm to design ultrawideband (UWB) pulse waveform, which was converted into a constraint problem with multiband chirp signals. Yongqiang et al. [252] investigated the receive antenna selection problem to maximize capacity in wireless MIMO communication system, which could be formulated as an integer programming optimization problem and could not be directly solved because of its nonconvex characteristics caused by the discrete binary antenna selection factor. Hence, they introduced PSO, in which the particle was defined as the discrete binary antenna selection factor and the objective function was associated with the capacity corresponding to the specified antenna subsection represented by the particle. Chiu et al. [253] presented a method for determining the required number and locations of transmitting antennas (TXs) to optimize wireless propagation coverage in an indoor UWB communication system. They used the $3 \mathrm{D}$ ray-tracing technique associated with a PSO and Asynchronous PSO (APSO) for optimizing the TXs location in an indoor environment. Minasian and Bird [254] applied PSO as a design tool for a parasitically coupled microstrip antenna array. The antenna was characterized by a unique nonintuitive design which resulted from an application of PSO with no constraints implemented on the shape of the array during optimization apart from the maximum dimensions. Zubair and Moinuddin [255] used PSO for joint optimization of three different shape-printed monopole antennas, namely, printed square monopole antenna, printed circular monopole antenna, and printed hexagonal monopole antenna, for UWB applications. Kim and Lee [256] applied metaheuristic to solve the optimum scheduling of multiple channels and time slots in multihop networks. SA and PSO were adopted to schedule the resources. The simulation results demonstrated that PSO-based scheduling outperformed SA-based scheduling in terms of end-to-end delay. Yazgan and Hakki Cavdar [257] comparatively studied PSO and least mean square (LMS) algorithms to estimate the optical communication channel parameters for radio over fiber systems. PSO reached better mean square error values, when selecting high noisy fiber optical channels or free space optical channels. Rabady and Ababneh [258] employed PSO to reach a global optimal design for optical interference filters, which were widely used in modern optical communication systems, biomedical applications, astronomy, and many others. Das et al. [259] applied ANN training with PSO for the problem of channel equalization. Existing applications of PSO to ANN training had only been used to find optimal weights of the network. Novelty in this paper was that it also took care of appropriate network topology and transfer functions of the neuron. The PSO algorithm optimized all the variables, including network weights and network parameters. Scott-Hayward and Garcia-Palacios [260] introduced a resource allocation solution capable of handling mixed media applications within the constraints of a $60 \mathrm{GHz}$ wireless network. A new channel time allocation PSO (CTA-PSO) was proposed to solve the network utility maximization resource allocation problem. CTA-PSO optimized the time allocated to each device in the network in order to maximize the quality of service experienced by each user. Omidvar and Mohammadi [261] used PSO in intelligent choosing of number of message copies. Regarding message delivery ratio and network overhead, PSO greatly helped in finding the suitable number of copies. Kuila and Jana [262] presented linear/nonlinear programming formulations of energy efficient clustering and routing problems for wireless sensor networks. They proposed two PSO-based algorithms to solve the optimization problem.

4.4. Operations Research. PSOs have been employed to solve problems in operations research. Liu and Wang [263] proposed a new task scheduling model. In the model, the task execution time was optimized in view of both the task running time and the system resource utilization. They improved the standard PSO and introduced a simple mutation mechanism and a self-adapting inertia weight method by classifying the fitness values. Che et al. [264] proposed a novel method based on the analytic network process and turbo PSO to evaluate partners and to determine an optimal supply chain network pattern and production-distribution mode. Hajipour and Pasandideh [265] focused on determination of the number of required facilities along with the relevant allocation process. They first proposed a novel biobjective facility location problem within batch arrival queuing framework under capacity, budget, and nearestfacility constraint. Then, PSO algorithm with considering a specific representation process was proposed to solve the biobjective problem. Cabrerizo et al. [266] used PSO to solve the granulation of the linguistic terms problem. A performance index was maximized by a suitable mapping of the linguistic terms on information granules. Prescilla and Selvakumar [267] applied a modified BPSO algorithm and a novel BPSO algorithm to solve the real-time task assignment in heterogeneous multiprocessor. The problem consisted of a set of independent periodic tasks, which had to be assigned to a heterogeneous multiprocessor without exceeding the utilization bound. The objective was to schedule maximum number of tasks with minimum energy consumption. Duan et al. [268] developed a multiple UCAVs cooperative air combat simulation platform, which was based on PSO, ACO, and game theory. The Matlab program was used as the developing tool. The practitioners could investigate the inherent mechanism by applying game theory to solve the mission decision-making problem of multiple UCAVs in attacking multiple objects. Belmecheri et al. [269] proposed a PSO with a local search to solve the vehicle routing problem (VRP) with heterogeneous fleet, mixed backhauls, and time windows. Hu et al. [270] proposed a hybrid chaos PSO (HPSO) to solve VRP with time window. The chaos algorithm was employed to reinitialize the particle swarm. 
An efficient insertion heuristic algorithm was also proposed to build the valid vehicle route in the particle decoding process. A premature convergence judgment mechanism was formulated and combined with the chaos algorithm and Gaussian mutation into HPSO when the particle swarm fell into the local convergence. Al Badawi and Shatnawi [271] developed an efficient method based on PSO to solve the multiprocessor task scheduling problem, which determined the assignment of tasks to the processors in a multiprocessor environment. Goksal et al. [272] presented a heuristic solution approach based on PSO, in which a local search was performed by variable neighborhood descent algorithm to solve the VRP with simultaneous pickup and delivery. Zhang et al. [273] first formulated and described the energyaware real-time task scheduling problem in heterogeneous multiprocessors. Then they proposed a PSO-based algorithm, which could successfully reduce the energy cost and the time for searching feasible solutions. Kechagiopoulos and Beligiannis [274] designed a PSO-based algorithm, aiming at the efficient solution of urban transit routing problem, which comprised an NP-hard problem that dealt with the construction of route networks for public transit networks.

4.5. Mechanical Engineering. A few applications of PSO are related to mechanical engineering. Ming et al. [275] investigated the implicit relationship between the compositions and mechanical properties of as-cast $\mathrm{Mg}$-Li-Al alloys. A momentum back-propagation (BP) neural network with a single hidden layer was established. PSO was applied to optimize the BP model. Chen et al. [276] aimed to develop a method for reliability-based optimum design of composite structures. A procedure combining PSO and finite element analysis (FEA) was proposed. Examples showed that the proposed method had good stability and was efficient in dealing with the probabilistic optimal design of composite structures. Mohan et al. [277] evaluated the use of frequency response function (FRF) with the help of PSO technique, for structural damage detection and quantification. It was observed that the use of FRF as response of damaged structure had led to better accuracy, since it contained data related to mode shape in addition to natural frequencies. Chen et al. [278] applied a surrogate based PSO algorithm, which combined the surrogate modeling technique and PSO, to the reliabilitybased robust design (RBRD) of composite pressure vessels. An optimization problem for maximizing the performance factor was formulated by choosing the winding orientation of the helical plies in the cylindrical portion, the thickness of metal liner, and the drop-off region size as the design variables. Strength constraints for composite layers and the metal liner were constructed by using Tsai-Wu failure criterion and Mises failure criterion, respectively. Zhang et al. [279] presented a methodology for the identification of parameter values in the Barcelona basic model (BBM) by inverse analysis of the experimental cavity pressure-cavity strain curve from pressure-meter tests in unsaturated soils. A novel parallel PSO algorithm was utilized to minimize the difference between measured and computed values on the cavity pressure-cavity strain curve. Wang et al. [280] proposed a decomposition-based multiobjective differential evolution PSO (DMDEPSO) algorithm for the design of a tubular permanent magnet linear synchronous motor (TPMLSM), which took into account multiple conflicting objectives. DMDEPSO hybridized DE and PSO together, first decomposed the multiobjective optimization problem into a number of single-objective optimization subproblems, each of which was associated with a Pareto optimal solution, and then optimized these subproblems simultaneously. Lazrag et al. [281] aimed to identify all the hydraulic parameters of sand by using a unique column test, which was a gravity drainage test where only the flow-rate temporal evolution was measured. They used several tensiometers installed in different positions along the column to measure the pressure head inside the soil sample. Inverse analysis was performed thanks to the PSO algorithm and the finite element modeling of the column test. Lee et al. [282] first developed 3D numerical models of a fractured femur with the locking compression plates (LCP). Then, the best screw position and number of LCPs were determined by using a simulationbased PSO algorithm, in order to discover the best number and positions of LCP screws to achieve acceptable fixation stability. Lake et al. [283] used PSO to optimize the geometry of a slotted MEMS resonator, in order to reduce energy dissipation from thermoelastic dissipation (TED). The optimization technique combined fundamental physics with PSO to navigate the complicated design space that arises from multiphysical problems. Vosoughi and Gerist [284] proposed a hybrid optimization technique that combines FEA, continuous GA, and PSO, for damage detection of laminated composite beams. Ribeiro et al. [285] investigated the geometric effects (thickness, width, and internal cell angle) of auxetic structures made of recycled rubber composites based on experimental and numerical data. The response surface models integrated with the PSO and FEA were proposed in order to obtain a range of solutions that provided useful information to the user during the selection of geometric parameters for reentrant cells. Kitak et al. [286] presented a method for determination of heat transfer coefficients for finite-element method housing model of medium-voltage switchgear cell. Suggested method was based on the PSO algorithm. A real test model of partition wall had been created as well as an equivalent numerical finite-element model. Kalatehjari et al. [287] applied PSO in 3D slope stability problem to determine the critical slip surface of soil slopes. In the contrast, past publications were limited to $2 \mathrm{D}$ slope stability analysis.

4.6. Civil Engineering. PSO was widely used to solve the optimization problems existing in civil engineering. Ashuri and Tavakolan [288] presented a hybrid GA-PSO approach to solve complex time-cost-resource optimization problem in construction project planning. The proposed method also used the fuzzy set theory to characterize uncertainty about the input data (i.e., time, cost, and resources required to perform an activity). Experiments showed that the proposed fuzzy enabled hybrid GA-PSO approach was superior to existing optimization algorithms at finding better project 
schedule solutions with less total project cost, less total project duration, and less total variation in resource allocation. Bozorgi-Amiri et al. [289] investigated a relief chain design problem where not only demands but also supplies and the cost of procurement and transportation were considered as the uncertain parameters. An efficient solution approach based on PSO was developed in order to solve the proposed mathematical model. Sadoghi Yazdi et al. [290] used a neurofuzzy model in conjunction with PSO for calibration of soil parameters used within a linear elastic-hardening plastic constitutive model with the Drucker-Prager yield criterion. The neurofuzzy system was used to provide a nonlinear regression between the deviatoric stress and axial strain obtained from a consolidated drained triaxial test on samples of poorly graded sand. The soil model parameters were determined in an iterative optimization loop with PSO and an adaptive network based on a fuzzy inference system such that the equations of the linear elastic model and (where appropriate) the hardening Drucker-Prager yielded criterion are simultaneously satisfied. Bolat et al. [291] developed a particle swarm optimization (PSO) algorithm to deal with car-call allocation problem. In vertical transportation, when a passenger makes a hall call by pressing a landing call button installed at the floor and located near the cars of the elevator group, the complex-elevator-group-control system must allocate one of the cars of the group to the hall call. Babu and Vijayalakshmi [292] presented a hybrid model PSO-GA, aimed at effectively utilizing local and global search capabilities of PSO and GA, respectively, to reduce the computational burden. Their analyses on different water distribution networks uncovered that the proposed hybrid model was capable of exploring the optimal combination of pipe diameters with minimal computational effort. Mohan et al. [293] showcased the efficacy of PSO and GA in damage assessment of structures, for early detection of cracks, corrosion, and structural failure in aging structures. The results showed the effectiveness of PSO in crack identification and the possibility of implementing it in a real-time structural health monitoring system for aircraft and civil structures. Asadnia et al. [294] presented the application of an improved PSO technique for training an ANN to predict water levels for the Heshui Watershed, China. The results indicated that the LM-NN model performed poorly in predicting the low and peak water levels in comparison to the PSO-based ANNs. Moreover, the IPSONN model was superior to CPSONN in predicting extreme water levels. Lastly, IPSONN had a quicker convergence rate compared to CPSONN. Yan et al. [295] presented a new imaging-based intelligent method for quantitatively rating the corrosion states of weathering steel bridges. To enhance the performance of a SVM in corrosion state classification, a PSO was developed to obtain the optimal parameters of the SVM. Their comparative study indicated that PSO-SVM could achieve better classification accuracy rates than ANN did. Wang and Li [296] advanced PSO and applied it to find effectively near-optimal solutions to the redundancy allocation problem of multistate systems with bridge topology, which was a commonly used structure for load balancing and control in applications such as electric power generation and transmission, transportation and computer networks, and electronic circuits. Sadeghi et al. [297] used an improved PSO to optimize a hybrid vendor-managed inventory and transportation problem with fuzzy demand. The parameters of both algorithms were calibrated using the Taguchi method to have better quality solutions. Kanović et al. [298] presented the comparison of some well-known global optimization techniques in optimization of an expert system controlling a ship locking process. The purpose was to find the best algorithm for optimization of membership function parameters of fuzzy expert system for the shiplock control. The results confirmed that all these procedures showed similar results and provided overall improvement of ship-lock operation performance.

4.7. Fuel and Energy. PSO had been introduced for the fuel and energy area. Mandal et al. [299] described the problem of short-term wind power production forecasting based on meteorological information. Aggregated wind power forecasts were produced for multiple wind farms using a hybrid intelligent algorithm that used a data filtering technique based on wavelet transform (WT) and a soft computing model based on neural network, which was optimized by using PSO algorithm. Chao [300] presented an adaptive maximum power point (MPP) tracking approach for PV power generation system. An optimization approach was proposed on the basis of a PSO algorithm for the complexity reduction in the determination of weighting values. Chen et al. [301] proposed chaotic improved PSO-based multiobjective optimization (MOCIPSO) and improved PSObased multiobjective optimization (MOIPSO) approaches, for solving complex multiobjective, mixed integer nonlinear problems such as minimization of power losses and $\mathrm{L}$ index in power systems simultaneously. Hu et al. [302] presented a data-driven method for estimating the capacity of Li-ion battery based on the charge voltage and current curves. An adaptation of PSO was proposed to find the optimal combination of feature weights for creating a $k \mathrm{NN}$ regression model that minimizes the cross validation (CV) error in the capacity estimation. Tabet et al. [303] used a mathematical model to determine the solar radiation incident on an inclined surface and the optimum slope angles for each month, season, and year are calculated for solar hybrid collectors. PSO method was applied to obtain the tilt angle setting of the tilt angle of photovoltaic thermal (PVT) hybrid collector. The objective was to improve the efficiency of PVT collector. Bahrami et al. [304] thought STLF (short-term electric load forecasting) played an important role in the operation of power systems. A new model based on combination of the WT and grey model (GM) was presented for STLF and was improved by PSO algorithm. Askarzadeh [305] studied the performance of different PSO variants for estimating Iran's electricity demand. Seven PSO variants, namely, original PSO, PSO-w (PSO with weighting factor), PSO-cf (PSO with constriction factor), PSO-rf (PSO with repulsion factor), PSO-vc (PSO with velocity control), CLPSO (comprehensive learning PSO), and a MPSO (modified PSO), were used to find the unknown weighting factors based on the data 
from 1982 to 2003. Sharafi and ElMekkawy [306] proposed a novel approach for optimal design of hybrid renewable energy systems (HRES) including various generators and storage devices. The epsilon-constraint method was applied to minimize simultaneously the total cost of the system, unmet load, and fuel emission. A PSO-simulation based approach was used to tackle the multiobjective optimization problem. García-Triviño et al. [307] presented and evaluated three energy management systems (EMSs) based on PSO for long-term operation optimization of a grid-connected hybrid system. It was composed of wind turbine (WT) and PV panels as primary energy sources and hydrogen system (fuel cell, electrolyzer and hydrogen storage tank) and battery as energy storage system (ESS). Biao et al. [308] proposed and established an electricity market based joint optimization scheduling model of thermal power plants, from the perspective of economics and environmental science. Mutation PSO (MPSO) was proposed to solve the model. Using an authentication instance, they compared and analyzed the performances of the MPSO method and the mixed integer programming method in solving the model. Chen et al. [309] proposed a sliding mode extremum seeking control (SMESC) of chaos embedded PSO (CEPSO) algorithm, applied to the design of MPP tracking in wind power systems. Its features were that the control parameters in SMESC were optimized by CEPSO, making it unnecessary to change the output power of different wind turbines, the designed in-repetition rate was reduced, and the system control efficiency was increased. Seyedmahmoudian et al. [310] aimed to employ PSO in MPP detection, while most conventional MPP tracking methods developed errors under certain circumstances and reduced the efficiency of PV systems even further. Aman et al. [311] presented a new approach for optimum simultaneous multiDG placement and sizing based on maximization of system loadability without violating the system constraints. DG penetration level, line limit, and voltage magnitudes were considered as system constraints. Hybrid PSO algorithm was also proposed to find the optimum solution considering maximization of system loadability and the corresponding minimum power losses. Xiao and Huang [312] proposed an optimization method combining PSO with FEA to design the heating system for rapid thermal cycling molding mold, aiming at high heating efficiency and uniform cavity surface temperature distribution. Lian et al. [313] proposed a MPP tracking method based on PSO in order to track the global maximum point. The proposed method was better than conventional MPP tracking methods such as perturb-andobserve method that could only track the first local maximum point and stop progressing to the next maximum point.

4.8. Medical Engineering. In medicine, Qasem and Shamsuddin [314] introduced a time variant MOPSO (TVMOPSO) of RBF network for diagnosing the medical diseases. The approach was tested on three standard datasets from UCI machine learning repository. Zhang et al. [315] developed an adaptive CPSO to train the parameters of feed-forward neural network, with the aim of accurate classification of magnetic resonance $(\mathrm{MR})$ brain images. Their results on 160 images showed the classification accuracy of the proposed method was $98.75 \%$. Guo et al. [316] proposed an adaptive PSO with neighborhood search to obtain the position and orientation of the medical microdevices. Experimental results showed that the tracking method was valid and the modified algorithm succeeded in dealing with the nonlinear system of equations in localization. Chang et al. [317] applied a PSO model to construct a decision-making system for diseases identification. The average forecasting accuracy for breast cancer was $97.4 \%$ and for liver disorders was $76.8 \%$. Chen et al. [318] proposed an analytical approach by integrating PSO and the 1-NN method. The proposed approach was applied to an actual case on the diagnosis of obstructive sleep apnea (OSA). Sung and Chiang [319] examined wireless sensor network with real-time remote identification using the Android study of things (HCIOT) platform in community healthcare. An improved PSO method was proposed to efficiently enhance physiological multisensors data fusion measurement precision in the Internet of Things (IOT) system. Cruz-Aceves et al. [320] presented a novel image segmentation method based on multiple active contours driven by PSO (MACPSO), which was used to segment the human heart and the human left ventricle from datasets of sequential computed tomography and MR images, respectively. Subasi [321] proposed a novel PSO-SVM model that hybridized the PSO and SVM in order to improve the electromyography (EMG) signal classification accuracy. Zhang et al. [322] proposed a novel hybrid system to classify a given MR brain image as either normal or abnormal. They used PSO to optimize the parameters $C$ and sigma, which were the key parameters of kernel SVM (KSVM). The results showed that the PSO was more effective to build optimal KSVM than random selection method. Mangat and Vig [323] discussed a rule mining classifier called DA-AC (dynamic adaptiveassociative classifier), which was based on a dynamic particle swarm optimizer. It could avoid premature convergence and provided a better value in every dimension. The method was then applied to predict life expectancy of postoperative thoracic surgery patients. Mandal et al. [324] formulated the fitting energy minimization problem to be solved using PSO technique. The algorithm was developed for two-phase level set implementation of the Chan and Vese model and it was successfully utilized for both scalar-valued and vectorvalued medical images. Hsieh et al. [325] proposed a class of hyperrectangular composite neural networks (HRCNNs) of which synaptic weights could be interpreted as a set of crisp If-Then rules and proposed a PSO-based fuzzy hyperrectangular composite neural network (PFHRCNN) which applied PSO to trim the rules generated by a trained HRCNN while the recognition performance would not be degraded or even be improved. Ganapathy et al. [326] proposed a new pattern classification system by combining temporal features with fuzzy min-max (TFMM) neural network based classifier for effective decision support in medical diagnosis. A PSO algorithm based rule extractor was also proposed for improving the detection accuracy.

4.9. Chemical Engineering. In chemistry, Thakral et al. [327] adopted PSO along with negative factor counting technique 
and inverse iteration method (IIM) for investigating the electronic properties of a model binary copolymer, of Type I class of quasi one-dimensional polymeric superlattices. Parastar et al. [328] proposed a multivariate curve resolution-PSO (MCR-PSO) algorithm to exploit pure chromatographic and spectroscopic information from multicomponent hyphenated chromatographic signals. Khajeh et al. [329] developed a simple and fast method for preconcentration and determination of trace amount of methylene blue from water samples by silver nanoparticles based solid-phase extraction method and UV-Vis spectrophotometry. Response surface methodology and hybrid of ANN-PSO were used to develop predictive models for simulation and optimization of solid-phase extraction method. Wu et al. [330] predicted $\mathrm{CO}_{2}$ solubility in polymers by RBF-ANN, based on a chaotic self-adaptive PSO (CSPSO) and fuzzy clustering method. The proposed method was also used to investigate solubility of $\mathrm{CO}_{2}$ in polystyrene, polypropylene, poly(butylene succinate), and poly(butylene succinate-co-adipate), respectively. Khajeh and Dastafkan [331] proposed a hybrid of ANN and PSO to develop predictive models for simulation and optimization of solidphase extraction method, with the aim of developing a simple and fast method for preconcentration and determination of trace amount of molybdenum from water samples by silver nanoparticles based solid-phase extraction method and UV-Vis spectrophotometry. Thakral et al. [332] investigated the electronic properties of the novel binary and ternary copolymers using PSO in combination with simple negative factor counting and inverse iteration method, using the ab initio Hartree Fock crystal orbital band structure results of the homopolymers, namely, polypyrrolo[3,4-C]pyrrole, polythieno[3,4-C]thiophene, and polyfurano[3,4-C]furan. Sun and Li [333] provided two improved mathematical expressions of attenuation function to quantify the effect of water in the process of methanol transformed to olefins on SAPO-34. Double PSO was employed to minimize the error objective function and the calculated values agree well with the experimental data. Skvortsov [334] researched on the rotation ambiguity (RA) in multivariate curve resolution (MCR), which was an undesirable case when the physicochemical constraints were not sufficiently strong to provide a unique resolution of the data matrix of the mixtures into spectra and concentration profiles of individual chemical components. They proposed a method to estimate RA with charged PSO (cPSO). cPSO-MCR was shown to be capable of estimating the strength of the constraints and of revealing RA in noisy data. Khansary and Sani [335] aimed for the prediction of liquid-liquid equilibria (LLE), Wilson, universal quasi chemical (UNIQUAC) and nonrandom two liquid (NRTL) models were used. Evolutionary algorithms such as GA and PSO were used for estimation of binary interaction parameters of these models. The reliability of GA and PSO in LLE applications was successfully approved. Nasimi and Irani [336] combined PSO with a BP algorithm to form a new learning algorithm for training artificial neural networks. This strategy was applied to model a highly nonlinear system of yeast fermentation bioreactor. Based on the results comparison, the PSO-BP model was found to be superior to the BP-ANN model in identification of nonlinear systems.
4.10. Biological Engineering. Saraswathi et al. [337] used a combination of integer-coded GA and PSO, coupled with the neural-network-based extreme learning machine (ELM), for gene selection and cancer classification. The proposed algorithm could handle sparse data and sample imbalance. Mansour et al. [338] presented a PSO-based algorithm for predicting protein structures in the $3 \mathrm{D}$ hydrophobic polar model. Starting from a small set of candidate solutions, their algorithm efficiently explored the search space and returns $3 \mathrm{D}$ protein structures with minimal energy. Karabulut and Ibrikci [339] proposed a PSO-based motif-finding method that utilized a proven Bayesian scoring scheme as the fitness function to identify transcription factor binding sites, which was a vital task in contemporary biology, since it helped researchers to comprehend the regulatory mechanism of gene expression. Liu et al. [340] established a classifier based on the two-layer PSO (TLPSO) algorithm and uncertain training sample sets. Samples of diffuse large B cell lymphoma (DLBLC) gene expression data were used for training and validating. The classification stability and accuracy by the proposed TLPSO algorithm increased significantly compared with the results obtained by using algorithms known as PSO and $K$-means. Salahi et al. [341] applied a variant of PSO with linear decreasing inertia weight to solve the problem of finding the global minimum of multifunnel-shaped functions with many local minima, which was a well-known and interesting problem in computational biology. Du et al. [342] presented construction of gene network using combined QPSO and K2 algorithm. Results showed QPSO-K2 algorithm performed better than stand-alone K2 and QPSO for all datasets. Chuang et al. [343] compared patients with high and low facial emotion perception (FEP) performances $(n=89$ and 93, resp.). A PSO algorithm was used to identify the best single-nucleotide polymorphisms (SNP) barcodes, that is, the SNP combinations and genotypes that revealed the largest differences between the high and low FEP groups. Mandal and Mukhopadhyay [344] organized the feature selection problem as a graph-theoretic problem where a feature-dissimilarity graph was shaped from the data matrix. They proposed a multiobjective BPSO that optimized average node-weight and average edge-weight of the candidate subgraph simultaneously. The proposed algorithm was applied for identifying relevant and nonredundant disease-related genes from microarray gene expression data. Chen et al. [345] developed a novel method utilizing PSO combined with a decision tree as the classifier to achieve efficient gene selection from thousands of candidate genes that could contribute in identifying cancers. Based on statistical analysis, the proposed method outperformed other popular classifiers for all test datasets and was compatible to SVM for certain specific datasets. García Nieto et al. [346] focused on turbidity parameter and how it was influenced by other water quality parameters in order to simplify water quality controls since they were expensive and time consuming. Turbidity values were predicted here by using the hybrid PSO-SVM-based model from the remaining measured water quality parameters (input variables) in the Nalon River basin (Northern Spain) with success. 
TABLE 3: Adherence to SI principles.

\begin{tabular}{ll}
\hline Principle & Adherence of PSO \\
\hline Proximity principle & Particle swarm carried out $N$-dimensional space calculation over a series of time steps. \\
Quality principle & Particle swarm responds to quality factors pbest \& gbest. \\
Diverse response principle & Particle swarm responses allocated between pbest and gbest. \\
Stability principle & Particle swarm changes state only when gbest changes. \\
Adaptability principle & Particle swarm changes state every time gbest changes. \\
\hline
\end{tabular}

\section{Discussion and Conclusion}

5.1. Adherence to SI Principles. Revisiting Section 2, we can find close adherence of PSO to the five SI principles by Millonas [15]. They can be expressed in Table 3. When the behavior of particle swarm explained below is reexamined, it is clear that the four principles of self-organization defined by Bonabeau et al. [14] are also fully satisfied.

5.2. Drawback, Improvement, and Innovation. Like all other SI-based optimization approaches, PSO has some drawbacks like premature, high computational complexity, slow convergence, sensitivity to parameters, and so forth. The reasons behind the problems are complicated. One potential reason is that PSO does not utilize the crossover operator as employed in GA or DE; hence the distribution of good information between candidates is not at a required level. Another reason may fall within the fact that PSO does not appropriately handle the relationship between exploitation (local search) and exploration (global search), so it usually converges to a local minimum quickly.

To address abovementioned problems, scholars have proposed many solutions listed in Section 3 (summarized in Table 4), which can be divided into following three types.

(i) Major modifications, including quantum-behaved PSO, bare-bones PSO, chaotic PSO, fuzzy PSO, PSOTVAC, OPSO, SPSO, and topology.

(ii) Minor modifications, including constriction coefficient, velocity clamping, trap detection, adaptive parameter, fitness-scaling, surrogate modeling, cooperative mechanism, boundary shifting, position resetting, entropy map, ecological behavior, jumping-out strategy, preference strategy, neighborhood learning, and local search.

(iii) Hybridization, PSO being hybridized with GA, SA, TS, AIS, ACO, HS, ABC, DE, and so forth.

Now there are over hundreds of both various PSO variants and test functions at present. It is impossible for each newly proposed PSO variant to compare with all other variants and to go through all test functions. Therefore, it is difficult to proclaim which type of modification is better or promising. In our opinion, it is important and essential to create a platform, to which the authors who proposed PSO variants can submit their programs. After the comprehensive and fair comparison, we then can conclude which PSO variant is the winner. Particularly, the design of PSO without parameters is worth studying.
Success of PSO as a single-objective optimizer for processing continuous optimization problem has motivated researchers to extend the use of PSO to other optimization fields, like multiobjective optimization, constrained optimization, and discrete optimization (binary optimization and combinatorial optimization).

(i) For multiobjective optimization, the optimizer needs to consider Pareto dominance every time it updates particles and store nondominated solutions to approximate the Pareto front.

(ii) For constrained optimization, both infeasible and feasible solutions should be generated at the search stage, and constraints are dealt with when evaluating solutions using penalty factor

(iii) For discrete optimization, a commonly used method is to map the discrete space to a continuous domain and apply standard PSO, followed by demapping the results. However, that method is not efficient and costs high computational resources. Particular PSObased methods have been developed as discrete PSO, binary PSO, and their variants.

Study of PSO in those fields had impressive achievements; however, lack of in-depth research on theoretical aspects impaired its application potentials. We believe it should be interesting to perform a more comprehensive theoretical study of both the run-time and convergence properties of PSO and its variants. Other aspects such as fitness landscapes and dynamics of PSO are also very attractive theoretical research directions.

For the programmers, writing parallel programs are more difficult than to write sequential programs, since they need to take into consideration the communication and synchronization between different subtasks. Therefore, there are merely a few publications related to PSO implemented in parallel. Some new computational forms are worth investigating within the next years, such as performing PSO in computer clusters, in grid computing, and in MPP (massively parallel processor).In addition to them, different computational levels of parallel computing are also interesting: bit-level, instruction-level, data-level, and task-level.

5.3. Trends of Applications. The first practical application of PSO was in the field of ANN training and was reported together with the algorithm itself [6]. Many areas of application were explored ever since. In addition to what we discussed in Section 4 (see Table 5), there are thousands of publications reporting application of PSO to other fields. Due to the page limit, we cannot list all of them. 
TABLE 4: Different modifications of PSO algorithm.

\begin{tabular}{|c|c|}
\hline Contributions & Studies \\
\hline QPSO & $\begin{array}{l}\text { Jau et al. [20], Jamalipour et al. [21], Bagheri et al. [22], Tang et al. [23], Davoodi et al. [24], Li and Xiao [25], } \\
\text { Yumin and Li [26], Jia et al. [27], and Gholizadeh and Moghadas [28] }\end{array}$ \\
\hline BBPSO & $\begin{array}{l}\text { Zhang et al. [30], Zhang et al. [31], Zhang et al. [32], Zhang et al. [33], Blackwell [34], Wang et al. [35], Jiang and } \\
\text { Wang [36], Liu et al. [37], Campos et al. [38], and Zhang et al. [39] }\end{array}$ \\
\hline CPSO & $\begin{array}{l}\text { Chuang et al. [40], Zhang and Wu [41], Dai et al. [42], Li et al. [43], Wu et al. [44], Zhang et al. [45], Zhang et al. } \\
\text { [46], Yang et al. [47], Son [48], He et al. [49], Zeng and Sun [50], and Pluhacek et al. [51] }\end{array}$ \\
\hline FPSO & $\begin{array}{l}\text { Juang et al. [52], Alfi and Fateh [53], Yang et al. [54], Norouzzadeh et al. [55], Robati et al. [56], Khan and } \\
\text { Engelbrecht [57], Galzina et al. [58], Nafar et al. [59], Aminian and Teshnehlab [60], and Chai et al. [61] }\end{array}$ \\
\hline PSOTVAC & $\begin{array}{l}\text { Cai et al. [62], Chaturvedi et al. [63], Boonyaritdachochai et al. [64], Sun et al. [65], Abedinia et al. [66], } \\
\text { Mohammadi-Ivatloo et al. [67], Mohammadi-Ivatloo et al. [68], Pookpunt and Ongsakul [69], Abedinia et al. } \\
\text { [70], Abdullah et al. [71], and Chih et al. [72] }\end{array}$ \\
\hline OPSO & $\begin{array}{l}\text { Dhahri and Alimi [73], Wang et al. [74], Dong et al. [75], Gao et al. [76], Khan et al. [77], Kaucic [78], Dai et al. } \\
\text { [79], and Muñoz et al. [80] }\end{array}$ \\
\hline SPSO & $\begin{array}{l}\text { Guochu [81], Pedersen and Chipperfield [82], Martins et al. [83], Panda et al. [84], Vastrakar and Padhy [85], } \\
\text { and Yeh [86] }\end{array}$ \\
\hline $\begin{array}{l}\text { Population } \\
\text { topology }\end{array}$ & $\begin{array}{l}\text { Wang and Watada [87], Jiang et al. [88], Marinakis and Marinaki [89], Rada-Vilela et al. [90], Wang et al. [91], } \\
\text { Fu et al. [92], Ni and Deng [93], Beheshti et al. [94], Lim and Isa [95], and Kalayci and Gupta [96] }\end{array}$ \\
\hline $\begin{array}{l}\text { Other } \\
\text { modifications }\end{array}$ & $\begin{array}{l}\text { Chuang et al. [97], Shi and Liu [98], Zhang et al. [99], Liu et al. [100], Shen et al. [101], Lin et al. [102], Wang and } \\
\text { Watada [103], Li et al. [104], Lu et al. [105], Mattos et al. [106], Wu et al. [107], Lim and Mat Isa [108], Shimizu et } \\
\text { al. [109], and Fister Jr. et al. [110] }\end{array}$ \\
\hline PSO-GA & $\begin{array}{l}\text { Kuo and Hong [111], Chen and Kurniawan [112], Nazir et al. [113], Vidhya and Kumar [114], Xiao et al. [115], and } \\
\text { Ghamisi and Benediktsson [116] }\end{array}$ \\
\hline PSO-AIS & Tang et al. [117], Zhang et al. [118], Ibrahim et al. [119], Kuo et al. [120], Liu et al. [121], and Darzi et al. [122] \\
\hline PSO-TS & Li et al. [123], Nakano et al. [124], Zhang et al. [125], Ktari and Chabchoub [126], and Wang et al. [127] \\
\hline PSO-ACO & $\begin{array}{l}\text { Chen and Chien [128], Xiao et al. [129], Kiran et al. [130], Huang et al. [131], Rahmani et al. [132], and Elloumi } \\
\text { [133] }\end{array}$ \\
\hline PSO-SA & $\begin{array}{l}\text { Sait et al. [134], Jiang and Zou [135], Niknam et al. [136], Khoshahval et al. [137], Du et al. [138], Zhang et al. } \\
\text { [139], and Geng et al. [140] }\end{array}$ \\
\hline PSO-ABC & El-Abd [141], Sharma et al. [142], Kiran and Gündüz [143], and Vitorino et al. [144] \\
\hline PSO-DE & $\begin{array}{l}\text { Maione and Punzi [145], Fu et al. [146], Vasundhara et al. [147], Yu et al. [148], Wang et al. [149], and Yadav and } \\
\text { Deep [150] }\end{array}$ \\
\hline $\begin{array}{l}\text { Other } \\
\text { hybridization }\end{array}$ & Xu et al. [151], Mohanty et al. [152], and Guo et al. [153] \\
\hline Multiple objective & $\begin{array}{l}\text { Qiu et al. [154], Chen et al. [155], Ghanei et al. [156], Duan et al. [157], Amiryousefi et al. [158], Ganguly [159], } \\
\text { Zhang et al. [160], Perera et al. [161], and Cheng et al. [162] }\end{array}$ \\
\hline $\begin{array}{l}\text { Constrained } \\
\text { optimization }\end{array}$ & $\begin{array}{l}\text { Daneshyari and Yen [163], Afshar [164], Koulinas et al. [165], Shan and Ren [166], Yeh and Chien [167], Singh et } \\
\text { al. [168], Paliwal et al. [169], Cui et al. [170], and Shou et al. [171] }\end{array}$ \\
\hline $\begin{array}{l}\text { Discrete } \\
\text { optimization }\end{array}$ & $\begin{array}{l}\text { Chen and Ludwig [172], Shen et al. [173], Chen et al. [174], Cai et al. [175], Kashan et al. [176], Xu et al. [177], } \\
\text { Garg and Singh [178], Zong et al. [179], and Ezzeldin et al. [180] }\end{array}$ \\
\hline $\begin{array}{l}\text { Binary } \\
\text { optimization }\end{array}$ & $\begin{array}{l}\text { Zhai and He [181], Sarath and Ravi [182], Taha and Abu Al Nadi [183], El-Maleh et al. [184], Erturk et al. [185], } \\
\text { Zhang et al. [186], Yin et al. [187], Yang et al. [188], and Ganesh et al. [189] }\end{array}$ \\
\hline Parameter choice & $\begin{array}{l}\text { Kumar and Chaturvedi [191], Zhang et al. [192], Yang [193], Sun et al. [194], Yassin et al. [195], Wang et al. [196], } \\
\text { Hao et al. [197], Xu [198], Chauhan et al. [199], Zhang et al. [200], and Kanemasa and Aiyoshi [201] }\end{array}$ \\
\hline $\begin{array}{l}\text { Convergence } \\
\text { analysis }\end{array}$ & $\begin{array}{l}\text { Wang and Shen [202], Sun et al. [203], Kurihara and Jin'no [204], Lin [205], Zhang et al. [206], Lin et al. [207], } \\
\text { and Kim and Li [208] }\end{array}$ \\
\hline Multicore & Waintraub et al. [209] and Yu [210] \\
\hline GPU & Hung and Wang [211], Rymut et al. [212], Kumar et al. [213], Awwad et al. [214], and Chen et al. [215] \\
\hline Cloud & Liu et al. [216], Xu and You [217], Ramezani et al. [218], Govindarajan et al. [219], and Ramezani et al. [220] \\
\hline
\end{tabular}


TABLE 5: Application areas of PSO algorithm.

\begin{tabular}{|c|c|}
\hline Area & Publication \\
\hline $\begin{array}{l}\text { Electrical and } \\
\text { electronic } \\
\text { engineering }\end{array}$ & $\begin{array}{l}\text { Ganguly et al. [221], Komsiyah [222], Feng et al. [223], Pekşen et al. [224], Yang et al. [225], de Mendonça et } \\
\text { al. [226], Liu et al. [227], Aich and Banerjee [228], Chou et al. [229], Lee et al. [230], Thakral and Bakhshi } \\
\text { [231], Fister et al. [232], Aghaei et al. [233], Selakov et al. [234], Shirvany et al. [235], and Tungadio et al. [236] }\end{array}$ \\
\hline Automatic control & $\begin{array}{l}\text { Cai and Yang [237], Kolomvatsos and Hadjieftymiades [238], Pandey et al. [239], Štimac et al. [240], Nedic et } \\
\text { al. [241], Chang and Chen [242], Xiang et al. [243], Danapalasingam [244], Mahmoodabadi et al. [245], } \\
\text { Zhong et al. [246], Perng et al. [247], Huang and Li [248], and Nisha and Pillai [249] }\end{array}$ \\
\hline Communication & $\begin{array}{l}\text { Yousefi et al. [250], Sun et al. [251], Yongqiang et al. [252], Chiu et al. [253], Zubair and Moinuddin [255], } \\
\text { Kim and Lee [256], Yazgan and Hakki Cavdar [257], Rabady and Ababneh [258], Das et al. [259], } \\
\text { Scott-Hayward and Garcia-Palacios [260], Omidvar and Mohammadi [261], and Kuila and Jana [262] }\end{array}$ \\
\hline Operations & $\begin{array}{l}\text { Liu and Wang [263], Che et al. [264], Hajipour and Pasandideh [265], Cabrerizo et al. [266], Prescilla and } \\
\text { Selvakumar [267], Duan et al. [268], Belmecheri et al. [269], Hu et al. [270], Al Badawia and Shatnawi [271], } \\
\text { Goksal et al. [272], Zhang et al. [273], and Kechagiopoulos and Beligiannis [274] }\end{array}$ \\
\hline $\begin{array}{l}\text { Mechanical } \\
\text { engineering }\end{array}$ & $\begin{array}{l}\text { Ming et al. [275], Chen et al. [276], Mohan et al. [277], Chen et al. [278], Zhang et al. [279], Wang et al. [280], } \\
\text { Lazrag et al. [281], Lee et al. [282], Lake et al. [283], Vosoughi and Gerist [284], Ribeiro et al. [285], Kitak et } \\
\text { al. [286], and Kalatehjari et al. [287] }\end{array}$ \\
\hline Civil engineering & $\begin{array}{l}\text { Ashuri and Tavakolan [288], Bozorgi-Amiri et al. [289], Sadoghi Yazdi et al. [290], Bolat et al. [291], Babu and } \\
\text { Vijayalakshmi [292], Mohan et al. [293], Asadnia et al. [294], Yan et al. [295], Wang and Li [296], Sadeghi et } \\
\text { al. [297], and Kanović et al. [298] }\end{array}$ \\
\hline Fuel and energy & $\begin{array}{l}\text { Mandal et al. [299], Chao [300], Chen et al. [301], Hu et al. [302], Tabet et al. [303], Bahrami et al. [304], } \\
\text { Askarzadeh [305], Sharafi and Elmekkawy [306], García-Triviño et al. [307], Biao et al. [308], Chen et al. } \\
\text { [309], Seyedmahmoudian et al. [310], Aman et al. [311], Xiao and Huang [312], and Lian et al. [313] }\end{array}$ \\
\hline Medicine engineering & $\begin{array}{l}\text { Qasem and Shamsuddin [314], Zhang et al. [315], Guo et al. [316], Chang et al. [317], Chen et al. [318], Sung } \\
\text { and Chiang [319], Cruz-Aceves et al. [320], Subasi [321], Zhang et al. [322], Mangat and Vig [323], Mandal et } \\
\text { al. [324], Hsieh et al. [325], and Ganapathy et al. [326] }\end{array}$ \\
\hline Chemical engineering & $\begin{array}{l}\text { Thakral et al. [327], Parastar et al. [328], Khajeh et al. [329], Wu et al. [330], Khajeh and Dastafkan [331], } \\
\text { Thakral et al. [332], Sun and Li [333], Skvortsova [334], Khansary and Sani [335], and Nasimi and Irani [336] }\end{array}$ \\
\hline $\begin{array}{l}\text { Biological } \\
\text { engineering }\end{array}$ & $\begin{array}{l}\text { Saraswathi et al. [337], Mansour et al. [338], Karabulut and Ibrikci [339], Liu et al. [340], Salahi et al. [341], } \\
\text { Du et al. [342], Chuang et al. [343], Mandal and Mukhopadhyay [344], Chen et al. [345], and García Nieto et } \\
\text { al. [346] }\end{array}$ \\
\hline
\end{tabular}

From the table, it is clear that the distribution of PSO exceeded our expectations. It is now applied to nearly every discipline that comes across optimization problems. Generally, PSO can be applied for optimization in dynamic and uncertain environments. The engineers need to first transform the problem to an optimization problem and then apply PSO to solve it.

Several research topics need to be fully investigated in future, since there are so far either no or merely few publications applying PSO to the optimization problem in those fields including what follows.

(i) Symbolic regression, which is a type of regression analysis that searches the space of mathematical expressions to find the model that best fits a given dataset, both in terms of accuracy and simplicity, is a crucially important theoretical and practical problem.

(ii) Floorplanning is to design the layout of equipment in a factory or components on a computer chip to reduce manufacturing time.

(iii) Weapon target assignment problem is to find an optimal assignment of a set of weapons of various types to a set of targets in order to maximize the expected damage done to the opponent. (iv) Supply chain management is the systemic, strategic coordination of the traditional business functions and the tactics across these business functions within a particular company and across businesses within the supply chain, for the purposes of improving the longterm performance of the individual companies and the supply chain as a whole [347].

(v) Nurse scheduling problem [348] is to find an optimal way to assign nurses to shifts, typically with a set of hard constraints in which all valid solutions must follow and a set of soft constraints which define the relative quality of valid solutions.

(vi) Queuing theory is the mathematical study of waiting lines. In queueing theory a model is constructed so that queue lengths and waiting times can be predicted to make business decisions about the resources needed [349].

5.4. Publication Analysis. Figure 3 demonstrated the number of publications related to PSO against year. It is clearly observed that the number of publications increases exponentially from 2000 to 2006, and then it fluctuates constantly and continually around about 1,000 publications per year from 2007 to 2013. We can conclude that the research in PSO has 


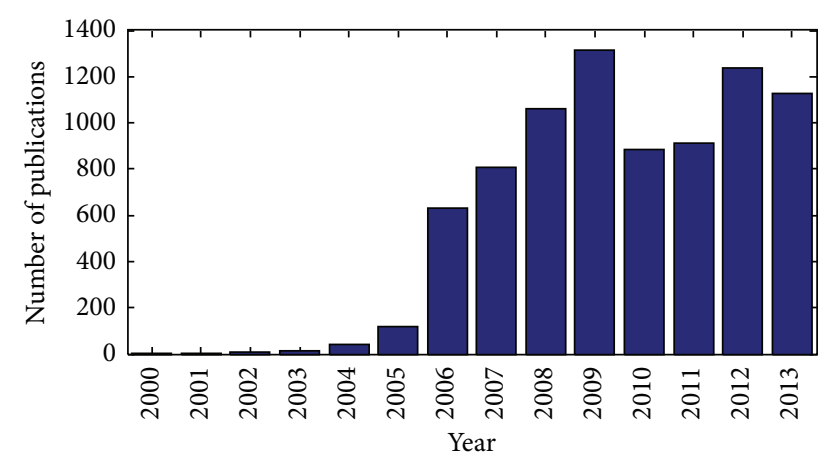

Figure 3: Publication number against year.

arrived at mature stage, and we expect a lot of more results within the coming few years.

\section{Acronyms}

ABC: Artificial bee colony

ACO: Ant colony optimization

AI: Artificial intelligence

AIS: Artificial immune system

ANN: Artificial neural network

BA: Bat algorithm

BBO: Biogeography-based optimization

BBPSO: Bare-bones PSO

BCI: Brain-computer interface

BFO: Bacterial foraging optimization

BP: Back-propagation

BPSO: Binary PSO

CI: $\quad$ Computational intelligence

CPSO: Chaotic PSO

CUDA: Compute unified device architecture

DCT: Discrete cosine transform

DE: Differential evolution

DG: Distributed generation

DPSO: Discrete PSO

EC: Evolutionary computation

EDP: Economic dispatch problem

EEG: Electroencephalography

ELD: Economic load dispatch

EMG: Electromyography

FCM: Fuzzy c-means

FEA: Finite element analysis

FPSO: Fuzzy PSO

GA: Genetic algorithm

GP: $\quad$ Genetic programming

GPU: Graphics processing unit

GSA: Gravitational search algorithm

GSO: Glowworm swarm optimization

HS: Harmonic search

HDFS: Hadoop distributed file system

KSVM: Kernel SVM

MIMO: Multivariable input and multivariable output

MLE: Maximum likelihood estimate

MOL: Many Optimizing Liaisons
MOPSO: Multiple objective PSO

MPP: $\quad$ Maximum power point

MR: $\quad$ Magnetic resonance

MRP: $\quad$ Multicast routing problem

MST: $\quad$ Minimal spanning tree

OBL: $\quad$ Opposition-based learning

OPSO: Opposition-based PSO

PID: $\quad$ Proportional-integral derivate

PSO: Particle swarm optimization

PSOTVAC: PSO with TVAC

PV: $\quad$ Photovoltaic

QPSO: Quantum-behaved PSO

RBF: Radial basis function

SA: $\quad$ Simulated annealing

SBS: $\quad$ Sequential backward selection

SFS: Sequential forward selection

SI: $\quad$ Swarm intelligence

SPSO: $\quad$ Simplified PSO

SQP: $\quad$ Sequential quadratic programming

SVM: $\quad$ Support vector machine

SVRM: Support vector regression machine

TS: $\quad$ Tabu search

TSP: $\quad$ Traveling salesman problem

TVAC: Time-varying acceleration coefficients

UAV: Unmanned aerial vehicle

UCAV: Unmanned combat aerial vehicle

UWB: Ultrawideband

VRP: $\quad$ Vehicle routing problem

WT: Wavelet transform.

\section{Conflict of Interests}

The authors declare that there is no conflict of interests regarding the publication of this paper.

\section{Acknowledgments}

This work was supported by NSFC (no. 610011024), Program of Natural Science Research of Jiangsu Higher Education Institutions of China (no. 14KJB520021), Jiangsu Key Laboratory of 3D Printing Equipment and Manufacturing (BM2013006), and Nanjing Normal University Research Foundation for Talented Scholars (no. 2013119XGQ0061).

\section{References}

[1] S. J. Russell and P. Norvig, Artificial Intelligence: A Modern Approach, Prentice Hall, New York, NY, USA, 2010.

[2] B. L. Agarwal, Basic Statistics, New Age International, 2006.

[3] K. E. Voges and N. Pope, Business Applications and Computational Intelligence, Idea Group Publishing, 2006.

[4] V. Kothari, J. Anuradha, S. Shah, and P. Mittal, "A survey on particle swarm optimization in feature selection," in Global Trends in Information Systems and Software Applications: Proceedings of the 4th International Conference, ObCom 2011, Vellore, TN, India, December 9-11, 2011, Part 2, P. V. Krishna, M. R. Babu, and E. Ariwa, Eds., vol. 270, pp. 192-201, Springer, Berlin, Germany, 2012. 
[5] M. Dorigo, Optimization, learning and natural algorithms [Ph.D. thesis], Politecnico di Milano, Milano, Italy, 1992.

[6] J. Kennedy and R. Eberhart, "Particle swarm optimization," in Proceedings of the IEEE International Conference on Neural Networks, vol. 4, pp. 1942-1948, December 1995.

[7] R. Storn and K. Price, "Differential evolution-a simple and efficient heuristic for global optimization over continuous spaces," Journal of Global Optimization, vol. 11, no. 4, pp. 341359, 1997.

[8] S. Müller, S. Airaghi, J. Marchetto, and P. Koumoutsakos, "Optimization algorithms based on a model of bacterial chemotaxis," in Proceedings of the 6th International Conference on Simulation of Adaptive Behavior: From Animals to Animats, pp. 375-384, 2000.

[9] K. M. Passino, "Biomimicry of bacterial foraging for distributed optimization and control," IEEE Control Systems Magazine, vol. 22, no. 3, pp. 52-67, 2002.

[10] D. Karaboga and B. Basturk, "Artificial Bee Colony (ABC) optimization algorithm for solving constrained optimization problems," in Proceedings of the Foundations of Fuzzy Logic and Soft Computing, P. Melin, O. Castillo, L. T. Aguilar, and W. Pedrycz, Eds., pp. 789-798, Springer, New York, NY, USA, 2007.

[11] K. N. Krishnanand and D. Ghose, "Detection of multiple source locations using a glowworm metaphor with applications to collective robotics," in Proceedings of the IEEE Swarm Intelligence Symposium (SIS '05), pp. 84-94, June 2005.

[12] X. S. Yang, "A new metaheuristic bat-inspired algorithm," in Nicso 2010: Nature Inspired Cooperative Strategies, J. R. Gonzalez, D. A. Pelta, C. Cruz, G. Terrazas, and N. Krasnogor, Eds., pp. 65-74, Springer, Berlin, Germany, 2010.

[13] Y. Zhang, P. Agarwal, V. Bhatnagar, S. Balochian, and X. Zhang, "Swarm intelligence and its applications 2014," The Scientific World Journal, vol. 2014, Article ID 204294, 4 pages, 2014.

[14] E. Bonabeau, M. Dorigo, and G. Theraulaz, Swarm Intelligence: From Natural to Artificial Systems, Oxford University Press, New York, NY, USA, 1999.

[15] M. Millonas, "Swarms, phase transitions and collective intelligence," in Artificial life III, C. Langton, Ed., pp. 417-445, Addison-Wesley, Reading, Mass, USA, 1994.

[16] Y. Zhang, S. Balochian, P. Agarwal, V. Bhatnagar, and O. J. Housheya, "Artificial intelligence and its applications," Mathematical Problems in Engineering, vol. 2014, Article ID 840491, 10 pages, 2014.

[17] F. Shahzad, S. Masood, and N. K. Khan, "Probabilistic opposition-based particle swarm optimization with velocity clamping," Knowledge and Information Systems, vol. 39, no. 3, pp. 703737, 2014.

[18] M. Clerc and J. Kennedy, "The particle swarm-explosion, stability, and convergence in a multidimensional complex space," IEEE Transactions on Evolutionary Computation, vol. 6, no. 1, pp. 58-73, 2002.

[19] Y. Zhang, S. Wang, and Z. Dong, "Classification of alzheimer disease based on structural magnetic resonance imaging by kernel support vector machine decision tree," Progress in Electromagnetics Research, vol. 144, pp. 171-184, 2014.

[20] Y.-M. Jau, K.-L. Su, C.-J. Wu, and J.-T. Jeng, "Modified quantumbehaved particle swarm optimization for parameters estimation of generalized nonlinear multi-regressions model based on Choquet integral with outliers," Applied Mathematics and Computation, vol. 221, pp. 282-295, 2013.
[21] M. Jamalipour, R. Sayareh, M. Gharib, F. Khoshahval, and M. R. Karimi, "Quantum behaved particle swarm optimization with differential mutation operator applied to WWER-1000 in-core fuel management optimization," Annals of Nuclear Energy, vol. 54, pp. 134-140, 2013.

[22] A. Bagheri, H. Mohammadi Peyhani, and M. Akbari, "Financial forecasting using ANFIS networks with Quantum-behaved Particle Swarm Optimization," Expert Systems with Applications, vol. 41, no. 14, pp. 6235-6250, 2014.

[23] D. Y. Tang, Y. Cai, J. Zhao, and Y. Xue, "A quantum-behaved particle swarm optimization with memetic algorithm and memory for continuous non-linear large scale problems," Information Sciences, vol. 289, pp. 162-189, 2014.

[24] E. Davoodi, M. T. Hagh, and S. G. Zadeh, "A hybrid improved quantum-behaved particle swarm optimizationsimplex method (IQPSOS) to solve power system load flow problems," Applied Soft Computing Journal, vol. 21, pp. 171-179, 2014.

[25] P. Li and H. Xiao, "An improved quantum-behaved particle swarm optimization algorithm," Applied Intelligence, vol. 40, no. 3, pp. 479-496, 2014.

[26] D. Yumin and Z. Li, "Quantum behaved particle swarm optimization algorithm based on artificial fish swarm," Mathematical Problems in Engineering, vol. 2014, Article ID 592682, 10 pages, 2014.

[27] P. F. Jia, F. C. Tian, S. Fan, Q. H. He, J. W. Feng, and S. X. Yang, "A novel sensor array and classifier optimization method of electronic nose based on enhanced quantum-behaved particle swarm optimization," Sensor Review, vol. 34, no. 3, pp. 304-311, 2014.

[28] S. Gholizadeh and R. K. Moghadas, "Performance-based optimum design of steel frames by an improved quantum particle swarm optimization," Advances in Structural Engineering, vol. 17, no. 2, pp. 143-156, 2014.

[29] J. Kennedy, "Bare bones particle swarms," in Proceedings of the IEEE Swarm Intelligence Symposium (SIS '03), pp. 80-87, Indianapolis, Ind, USA, 2003.

[30] H. Zhang, D. D. Kennedy, G. P. Rangaiah, and A. BonillaPetriciolet, "Novel bare-bones particle swarm optimization and its performance for modeling vapor-liquid equilibrium data," Fluid Phase Equilibria, vol. 301, no. 1, pp. 33-45, 2011.

[31] J. Q. Zhang, L. Ni, J. Yao, W. Wang, and Z. Tang, "Adaptive bare bones particle swarm inspired by cloud model," IEICE Transactions on Information and Systems, vol. 94, no. 8, pp. 15271538, 2011.

[32] H. Zhang, J. A. Fernández-Vargas, G. P. Rangaiah, A. BonillaPetriciolet, and J. G. Segovia-Hernández, "Evaluation of integrated differential evolution and unified bare-bones particle swarm optimization for phase equilibrium and stability problems," Fluid Phase Equilibria, vol. 310, no. 1-2, pp. 129-141, 2011.

[33] Y. Zhang, D.-W. Gong, and Z. Ding, "A bare-bones multiobjective particle swarm optimization algorithm for environmental/economic dispatch," Information Sciences, vol. 192, pp. 213-227, 2012.

[34] T. Blackwell, "A study of collapse in bare bones particle swarm optimization," IEEE Transactions on Evolutionary Computation, vol. 16, no. 3, pp. 354-372, 2012.

[35] P. P. Wang, L. Shi, Y. Zhang, and L. Han, "A hybrid simplex search and modified bare-bones particle swarm optimization," Chinese Journal of Electronics, vol. 22, no. 1, pp. 104-108, 2013. 
[36] B. Jiang and N. Wang, "Cooperative bare-bone particle swarm optimization for data clustering," Soft Computing, vol. 18, no. 6, pp. 1079-1091, 2014.

[37] H. Liu, G. Ding, and B. Wang, "Bare-bones particle swarm optimization with disruption operator," Applied Mathematics and Computation, vol. 238, pp. 106-122, 2014.

[38] M. Campos, R. A. Krohling, and I. Enriquez, "Bare bones particle swarm optimization with scale matrix adaptation," IEEE Transactions on Cybernetics, vol. 44, no. 9, pp. 1567-1578, 2014.

[39] Y. Zhang, D. Gong, Y. Hu, and W. Zhang, "Feature selection algorithm based on bare bones particle swarm optimization," Neurocomputing, vol. 148, pp. 150-157, 2015.

[40] L.-Y. Chuang, S.-W. Tsai, and C.-H. Yang, "Chaotic catfish particle swarm optimization for solving global numerical optimization problems," Applied Mathematics and Computation, vol. 217, no. 16, pp. 6900-6916, 2011.

[41] Y. Zhang and L. Wu, "Crop classification by forward neural network with adaptive chaotic particle swarm optimization," Sensors, vol. 11, no. 5, pp. 4721-4743, 2011.

[42] Y. S. Dai, Y. Wei, J. Chen, Y. Zhang, and J. Ding, "Seismic wavelet estimation based on adaptive chaotic embedded particle swarm optimization algorithm," in Proceedings of the 5th International Symposium on Computational Intelligence and Design (ISCID '12), vol. 2, pp. 57-60, October 2012.

[43] C. S. Li, J. Zhou, P. Kou, and J. Xiao, "A novel chaotic particle swarm optimization based fuzzy clustering algorithm," Neurocomputing, vol. 83, pp. 98-109, 2012.

[44] Q. Wu, R. Law, E. Wu, and J. Lin, "A hybrid-forecasting model reducing Gaussian noise based on the Gaussian support vector regression machine and chaotic particle swarm optimization," Information Sciences, vol. 238, pp. 96-110, 2013.

[45] Y. Zhang, L. Wu, and S. Wang, "UCAV path planning by fitness-scaling adaptive chaotic particle swarm optimization," Mathematical Problems in Engineering, vol. 2013, Article ID 705238, 9 pages, 2013.

[46] Q. Zhang, Z. Li, C. J. Zhou, and X. P. Wei, "Bayesian network structure learning based on the chaotic particle swarm optimization algorithm," Genetics and Molecular Research, vol. 12, no. 4, pp. 4468-4479, 2013.

[47] C.-H. Yang, Y.-D. Lin, L.-Y. Chuang, and H.-W. Chang, "Double-bottom chaotic map particle swarm optimization based on chi-square test to determine gene-gene interactions," BioMed Research International, vol. 2014, Article ID 172049, 10 pages, 2014.

[48] L. H. Son, "Optimizing municipal solid waste collection using chaotic particle swarm optimization in GIS based environments: a case study at Danang city, Vietnam," Expert Systems with Applications, vol. 41, no. 18, pp. 8062-8074, 2014.

[49] H.-D. He, W.-Z. Lu, and Y. Xue, "Prediction of particulate matter at street level using artificial neural networks coupling with chaotic particle swarm optimization algorithm," Building and Environment, vol. 78, pp. 111-117, 2014.

[50] Y. J. Zeng and Y. G. Sun, "An improved particle swarm optimization for the combined heat and power dynamic economic dispatch problem," Electric Power Components and Systems, vol. 42, no. 15, pp. 1700-1716, 2014.

[51] M. Pluhacek, R. Senkerik, and I. Zelinka, "Particle swarm optimization algorithm driven by multichaotic number generator," Soft Computing, vol. 18, no. 4, pp. 631-639, 2014.
[52] Y.-T. Juang, S.-L. Tung, and H.-C. Chiu, "Adaptive fuzzy particle swarm optimization for global optimization of multimodal functions," Information Sciences, vol. 181, no. 20, pp. 4539-4549, 2011.

[53] A. Alfi and M.-M. Fateh, "Intelligent identification and control using improved fuzzy particle swarm optimization," Expert Systems with Applications, vol. 38, no. 10, pp. 12312-12317, 2011.

[54] W.-A. Yang, Y. Guo, and W.-H. Liao, "Optimization of multipass face milling using a fuzzy particle swarm optimization algorithm," International Journal of Advanced Manufacturing Technology, vol. 54, no. 1-4, pp. 45-57, 2011.

[55] M. S. Norouzzadeh, M. R. Ahmadzadeh, and M. Palhang, "LADPSO: using fuzzy logic to conduct PSO algorithm," Applied Intelligence, vol. 37, no. 2, pp. 290-304, 2012.

[56] A. Robati, G. A. Barani, H. Nezam Abadi Pour, M. J. Fadaee, and J. R. Anaraki, "Balanced fuzzy particle swarm optimization," Applied Mathematical Modelling, vol. 36, no. 5, pp. 2169-2177, 2012.

[57] S. A. Khan and A. P. Engelbrecht, "A fuzzy particle swarm optimization algorithm for computer communication network topology design," Applied Intelligence, vol. 36, no. 1, pp. 161-177, 2012.

[58] V. Galzina, R. Lujić, and T. Šarić, "Adaptive fuzzy particle swarm optimization for flow-shop scheduling problem," Tehnicki Vjesnik-Technical Gazette, vol. 19, no. 1, pp. 151-157, 2012.

[59] M. Nafar, G. B. Gharehpetian, and T. Niknam, "Using modified fuzzy particle swarm optimization algorithm for parameter estimation of surge arresters models," International Journal of Innovative Computing, Information and Control, vol. 8, no. 1, pp. 567-581, 2012.

[60] E. Aminian and M. Teshnehlab, "A novel fuzzy particle swarm optimization," in Proceedings of the 13th Iranian Conference on Fuzzy Systems, pp. 1-6, IEEE, Qazvin, Iran, 2013.

[61] R. Chai, S. H. Ling, G. P. Hunter, Y. Tran, and H. T. Nguyen, "Brain-computer interface classifier for wheelchair commands using neural network with fuzzy particle swarm optimization," IEEE Journal of Biomedical and Health Informatics, vol. 18, no. 5, pp. 1614-1624, 2014.

[62] X. J. Cai, Y. Cui, and Y. Tan, "Predicted modified PSO with timevarying accelerator coefficients," International Journal of BioInspired Computation, vol. 1, no. 1-2, pp. 50-60, 2009.

[63] K. T. Chaturvedi, M. Pandit, and L. Srivastava, "Particle swarm optimization with time varying acceleration coefficients for non-convex economic power dispatch," International Journal of Electrical Power \& Energy Systems, vol. 31, no. 6, pp. 249-257, 2009.

[64] P. Boonyaritdachochai, C. Boonchuay, and W. Ongsakul, "Optimal congestion management in an electricity market using particle swarm optimization with time-varying acceleration coefficients," Computers \& Mathematics with Applications, vol. 60, no. 4, pp. 1068-1077, 2010.

[65] C. Y. Sun, H. N. Zhao, and Y. F. Wang, "A comparative analysis of PSO, HPSO, and HPSO-TVAC for data clustering," Journal of Experimental and Theoretical Artificial Intelligence, vol. 23, no. 1, pp. 51-62, 2011.

[66] O. Abedinia, N. Amjady, and K. Kiani, "Optimal complex economic load dispatch solution using particle swarm optimization with time varying acceleration coefficient," International Review of Electrical Engineering, vol. 7, no. 2, pp. 4249-4256, 2012.

[67] B. Mohammadi-Ivatloo, A. Rabiee, A. Soroudi, and M. Ehsan, "Iteration PSO with time varying acceleration coefficients for 
solving non-convex economic dispatch problems," International Journal of Electrical Power \& Energy Systems, vol. 42, no. 1, pp. 508-516, 2012.

[68] B. Mohammadi-Ivatloo, M. Moradi-Dalvand, and A. Rabiee, "Combined heat and power economic dispatch problem solution using particle swarm optimization with time varying acceleration coefficients," Electric Power Systems Research, vol. 95, pp. 9-18, 2013.

[69] S. Pookpunt and W. Ongsakul, "Optimal placement of wind turbines within wind farm using binary particle swarm optimization with time-varying acceleration coefficients," Renewable Energy, vol. 55, pp. 266-276, 2013.

[70] O. Abedinia, N. Amjady, A. Ghasemi, and Z. Hejrati, "Solution of economic load dispatch problem via hybrid particle swarm optimization with time-varying acceleration coefficients and bacteria foraging algorithm techniques," International Transactions on Electrical Energy Systems, vol. 23, no. 8, pp. 1504-1522, 2013.

[71] M. N. Abdullah, A. H. A. Bakar, N. A. Rahim, H. Mokhlis, H. A. Illias, and J. J. Jamian, "Modified particle swarm optimization with time varying acceleration coefficients for economic load dispatch with generator constraints," Journal of Electrical Engineering \& Technology, vol. 9, no. 1, pp. 15-26, 2014.

[72] M. C. Chih, C.-J. Lin, M.-S. Chern, and T.-Y. Ou, "Particle swarm optimization with time-varying acceleration coefficients for the multidimensional knapsack problem," Applied Mathematical Modelling, vol. 38, no. 4, pp. 1338-1350, 2014.

[73] H. Dhahri and A. M. Alimi, "Opposition-based particle swarm optimization for the design of beta basis function neural network," in Proceedings of the International Joint Conference on Neural Networks (IJCNN '10), pp. 1-8, 2010.

[74] H. Wang, Z. Wu, S. Rahnamayan, Y. Liu, and M. Ventresca, "Enhancing particle swarm optimization using generalized opposition-based learning," Information Sciences, vol. 181, no. 20, pp. 4699-4714, 2011.

[75] N. Dong, C.-H. Wu, W.-H. Ip, Z.-Q. Chen, C.-Y. Chan, and K.-L. Yung, "An opposition-based chaotic GA/PSO hybrid algorithm and its application in circle detection," Computers \& Mathematics with Applications, vol. 64, no. 6, pp. 1886-1902, 2012.

[76] W. F. Gao, S. Y. Liu, and L. L. Huang, "Particle swarm optimization with chaotic opposition-based population initialization and stochastic search technique," Communications in Nonlinear Science and Numerical Simulation, vol. 17, no. 11, pp. 4316-4327, 2012.

[77] N. K. Khan, A. R. Baig, and M. A. Iqbal, "Opposition-based discrete PSO using natural encoding for classification rule discovery," International Journal of Advanced Robotic Systems, vol. 9, 2012.

[78] M. Kaucic, "A multi-start opposition-based particle swarm optimization algorithm with adaptive velocity for bound constrained global optimization," Journal of Global Optimization, vol. 55, no. 1, pp. 165-188, 2013.

[79] Y. Dai, L. Liu, and S. Feng, "On the identification of coupled pitch and heave motions using opposition-based particle swarm optimization," Mathematical Problems in Engineering, vol. 2014, Article ID 784049, 10 pages, 2014.

[80] D. M. Muñoz, C. H. Llanos, L. D. S. Coelho, and M. AyalaRincón, "Hardware opposition-based PSO applied to mobile robot controllers," Engineering Applications of Artificial Intelligence, vol. 28, pp. 64-77, 2014.
[81] C. Guochu, "Simplified particle swarm optimization algorithm based on particles classification," in Proceedings of the 6th International Conference on Natural Computation (ICNC'10), pp. 2701-2705, August 2010.

[82] M. E. H. Pedersen and A. J. Chipperfield, "Simplifying particle swarm optimization," Applied Soft Computing Journal, vol. 10, no. 2, pp. 618-628, 2010.

[83] C. H. Martins, R. P. B. dos Santos, and F. L. Santos, "Simplified particle swarm optimization algorithm," Acta ScientiarumTechnology, vol. 34, no. 1, pp. 21-25, 2012.

[84] S. Panda, B. K. Sahu, and P. K. Mohanty, "Design and performance analysis of PID controller for an automatic voltage regulator system using simplified particle swarm optimization," Journal of the Franklin Institute, vol. 349, no. 8, pp. 2609-2625, 2012.

[85] N. K. Vastrakar and P. K. Padhy, "Simplified PSO PI-PD controller for unstable processes," in Proceedings of the 4th International Conference on Intelligent Systems, Modelling o Simulation (ISMS '13), pp. 350-354, IEEE, Bangkok, Thailand, January 2013.

[86] W.-C. Yeh, "New parameter-free simplified swarm optimization for artificial neural network training and its application in the prediction of time series," IEEE Transactions on Neural Networks and Learning Systems, vol. 24, no. 4, pp. 661-665, 2013.

[87] S. Wang and J. M. Watada, "Two-stage fuzzy stochastic programming with Value-at-Risk criteria," Applied Soft Computing Journal, vol. 11, no. 1, pp. 1044-1056, 2011.

[88] B. Jiang, N. Wang, and L. P. Wang, "Particle swarm optimization with age-group topology for multimodal functions and data clustering," Communications in Nonlinear Science and Numerical Simulation, vol. 18, no. 11, pp. 3134-3145, 2013.

[89] Y. Marinakis and M. Marinaki, "Particle swarm optimization with expanding neighborhood topology for the permutation flowshop scheduling problem," Soft Computing, vol. 17, no. 7, pp. 1159-1173, 2013.

[90] J. Rada-Vilela, M. J. Zhang, and W. Seah, "A performance study on synchronicity and neighborhood size in particle swarm optimization," Soft Computing, vol. 17, no. 6, pp. 1019-1030, 2013.

[91] H. Wang, H. Sun, C. Li, S. Rahnamayan, and J.-S. Pan, "Diversity enhanced particle swarm optimization with neighborhood search," Information Sciences, vol. 223, pp. 119-135, 2013.

[92] X. Fu, W. Liu, B. Zhang, and H. Deng, "Quantum behaved particle swarm optimization with neighborhood search for numerical optimization," Mathematical Problems in Engineering, vol. 2013, Article ID 469723, 10 pages, 2013.

[93] Q. J. Ni and J. M. Deng, "A new logistic dynamic particle swarm optimization algorithm based on random topology," The Scientific World Journal, vol. 2013, Article ID 409167, 8 pages, 2013.

[94] Z. Beheshti, S. M. Shamsuddin, and S. Sulaiman, "Fusion global-local-topology particle swarm optimization for global optimization problems," Mathematical Problems in Engineering, vol. 2014, Article ID 907386, 19 pages, 2014.

[95] W. H. Lim and N. A. M. Isa, "Particle swarm optimization with increasing topology connectivity," Engineering Applications of Artificial Intelligence, vol. 27, pp. 80-102, 2014.

[96] C. B. Kalayci and S. M. Gupta, "A particle swarm optimization algorithm with neighborhood-based mutation for sequencedependent disassembly line balancing problem," International Journal of Advanced Manufacturing Technology, vol. 69, no. 1-4, pp. 197-209, 2013. 
[97] L.-Y. Chuang, S.-W. Tsai, and C.-H. Yang, "Catfish particle swarm optimization," in Proceedings of the IEEE Swarm Intelligence Symposium (SIS '08), IEEE, St. Louis, Mo, USA, September 2008.

[98] R. F. Shi and X. J. Liu, "A hybrid improved particle swarm optimization based on dynamic parameters control and metropolis accept rule strategy," in Proceedings of the 3rd International Conference on Genetic and Evolutionary Computing (WGEC '09), T. L. Huang, L. F. Li, and M. Zhao, Eds., pp. 649-653, IEEE Computer Society, Los Alamitos, Calif, USA, October 2009.

[99] J. H. Zhang, Y. Wang, R. Wang, and G. Hou, "Bidding strategy based on adaptive particle swarm optimization for electricity market," in Proceedings of the 8th World Congress on Intelligent Control and Automation (WCICA '10), pp. 3207-3210, IEEE, Jinan, China, July 2010.

[100] A. Liu, Y. Yang, Q. Xing, H. Yao, Y. Zhang, and Z. Zhou, "Improved collaborative particle swarm algorithm for job shop scheduling optimization," Advanced Science Letters, vol. 4, no. 6-7, pp. 2180-2183, 2011.

[101] Y. X. Shen, G. Y. Wang, and C. M. Tao, "Particle swarm optimization with novel processing strategy and its application," International Journal of Computational Intelligence Systems, vol. 4, no. 1, pp. 100-111, 2011.

[102] L. Lin, Z. Ji, S. He, and Z. Zhu, "A crown jewel defense strategy based particle swarm optimization," in Proceedings of the IEEE Congress on Evolutionary Computation (CEC '12), June 2012.

[103] S. M. Wang and J. Watada, "A hybrid modified PSO approach to VaR-based facility location problems with variable capacity in fuzzy random uncertainty," Information Sciences, vol. 192, pp. 3-18, 2012.

[104] Z. Li, Z. Tian, Y. Xie, R. Huang, and J. Tan, "A knowledgebased heuristic particle swarm optimization approach with the adjustment strategy for the weighted circle packing problem," Computers \& Mathematics with Applications, vol. 66, no. 10, pp. 1758-1769, 2013.

[105] Y. C. Lu, J. C. Jan, S. L. Hung, and G. H. Hung, "Enhancing particle swarm optimization algorithm using two new strategies for optimizing design of truss structures," Engineering Optimization, vol. 45, no. 10, pp. 1251-1271, 2013.

[106] C. L. C. Mattos, G. A. Barreto, and F. R. P. Cavalcanti, "An improved hybrid particle swarm optimization algorithm applied to economic modeling of radio resource allocation," Electronic Commerce Research, vol. 14, no. 1, pp. 51-70, 2014.

[107] G. Wu, W. Pedrycz, M. Ma, D. Qiu, H. Li, and J. Liu, "A particle swarm optimization variant with an inner variable learning strategy," The Scientific World Journal, vol. 2014, Article ID 713490, 15 pages, 2014.

[108] W. H. Lim and N. A. Mat Isa, "An adaptive two-layer particle swarm optimization with elitist learning strategy," Information Sciences, vol. 273, pp. 49-72, 2014.

[109] Y. Shimizu, T. Sakaguchi, and T. Miura, "Parallel computing for huge scale logistics optimization through binary PSO associated with topological comparison," Journal of Advanced Mechanical Design, Systems, and Manufacturing, vol. 8, no. 1, Article ID JAMDSM0005, 2014.

[110] I. Fister Jr., M. Perc, K. Ljubič, S. M. Kamal, and A. Iglesias, "Particle swarm optimization for automatic creation of complex graphic characters," Chaos, Solitons \& Fractals, vol. 73, pp. 2935, 2015.
[111] R. J. Kuo and C. W. Hong, "Integration of genetic algorithm and particle swarm optimization for investment portfolio optimization," Applied Mathematics and Information Sciences, vol. 7, no. 6, pp. 2397-2408, 2013.

[112] W. C. Chen and D. Kurniawan, "Process parameters optimization for multiple quality characteristics in plastic injection molding using Taguchi method, BPNN, GA, and hybrid PSOGA," International Journal of Precision Engineering and Manufacturing, vol. 15, no. 8, pp. 1583-1593, 2014.

[113] M. Nazir, A. Majid-Mirza, and S. Ali-Khan, "PSO-GA based optimized feature selection using facial and clothing information for gender classification," Journal of Applied Research and Technology, vol. 12, no. 1, pp. 145-152, 2014.

[114] K. Vidhya and K. R. S. Kumar, "Channel estimation of MIMOOFDM system using PSO and GA," Arabian Journal for Science and Engineering, vol. 39, no. 5, pp. 4047-4056, 2014.

[115] Y. Xiao, J. Xiao, F. Lu, and S. Wang, "Ensemble ANNs-PSO-GA approach for day-ahead stock E-exchange prices forecasting," International Journal of Computational Intelligence Systems, vol. 7, no. 2, pp. 272-290, 2014.

[116] P. Ghamisi and J. A. Benediktsson, "Feature selection based on hybridization of genetic algorithm and particle swarm optimization," IEEE Geoscience and Remote Sensing Letters, vol. 12, no. 2, pp. 309-313, 2015.

[117] H. Z. Tang, Y. Xiao, H. Huang, and X. Guo, "A novel dynamic particle swarm optimization algorithm based on improved artificial immune network," in Proceedings of the 10th IEEE International Conference on Signal Processing (ICSP '10), B. Z. Yuan, Q. Q. Ruan, and X. F. Tang, Eds., pp. 103-106, New York, NY, USA, October 2010.

[118] Y. Zhang, Y. Jun, G. Wei, and L. Wu, "Find multi-objective paths in stochastic networks via chaotic immune PSO," Expert Systems with Applications, vol. 37, no. 3, pp. 1911-1919, 2010.

[119] A. A. Ibrahim, A. Mohamed, H. Shareef, and S. P. Ghoshal, "Optimal power quality monitor placement in power systems based on particle swarm optimization and artificial immune system," in Proceedings of the 3rd Conference on Data Mining and Optimization (DMO '11), pp. 141-145, June 2011.

[120] R. J. Kuo, C. M. Chen, T. W. Liao, and F. C. Tien, "Hybrid of artificial immune system and particle swarm optimization-based support vector machine for radio frequency identificationbased positioning system," Computers and Industrial Engineering, vol. 64, no. 1, pp. 333-341, 2013.

[121] Z.-H. Liu, J. Zhang, S.-W. Zhou, X.-H. Li, and K. Liu, "Coevolutionary particle swarm optimization using AIS and its application in multiparameter estimation of PMSM," IEEE Transactions on Cybernetics, vol. 43, no. 6, pp. 1921-1935, 2013.

[122] S. Darzi, T. S. Kiong, M. T. Islam, M. Ismail, S. Kibria, and B. Salem, "Null steering of adaptive beamforming using linear constraint minimum variance assisted by particle swarm optimization," The Scientific World Journal, vol. 2014, Article ID 724639, 10 pages, 2014.

[123] Z. C. Li, D. J. Zheng, and H. J. Hou, "A hybrid particle swarm optimization algorithm based on nonlinear simplex method and tabu search," in Advances in Neural NetworksISNN 2010, L. Q. Zhang, B. L. Lu, and J. Kwok, Eds., vol. 6063 of Lecture Notes in Computer Science, pp. 126-135, Springer, Berlin, Germany, 2010.

[124] S. Nakano, A. Ishigame, and K. Yasuda, "Consideration of particle swarm optimization combined with tabu search," Electrical Engineering in Japan, vol. 172, no. 4, pp. 31-37, 2010. 
[125] T. Zhang, Y. J. Zhang, Q. P. Zheng, and P. M. Pardalos, "A hybrid particle swarm optimization and tabu seach algorithm for order planning problems of steel factories based on the make-tostock and make-to-order management architecture," Journal of Industrial and Management Optimization, vol. 7, no. 1, pp. 31-51, 2011.

[126] R. Ktari and H. Chabchoub, "Essential particle swarm optimization queen with tabu search for MKP resolution," Computing, vol. 95, no. 9, pp. 897-921, 2013.

[127] J. Wang, J. Lu, Z. Bie, S. You, and X. Cao, "Long-term maintenance scheduling of smart distribution system through a PSOTS algorithm," Journal of Applied Mathematics, vol. 2014, Article ID 694086, 12 pages, 2014.

[128] S.-M. Chen and C.-Y. Chien, "Solving the traveling salesman problem based on the genetic simulated annealing ant colony system with particle swarm optimization techniques," Expert Systems with Applications, vol. 38, no. 12, pp. 14439-14450, 2011.

[129] R. Xiao, W. Chen, and T. Chen, "Modeling of ant colony's labor division for the multi-project scheduling problem and its solution by pso," Journal of Computational and Theoretical Nanoscience, vol. 9, no. 2, pp. 223-232, 2012.

[130] M. S. Kiran, E. Özceylan, M. Gündüz, and T. Paksoy, "A novel hybrid approach based on Particle Swarm Optimization and Ant Colony Algorithm to forecast energy demand of Turkey," Energy Conversion and Management, vol. 53, no. 1, pp. 75-83, 2012.

[131] C.-L. Huang, W.-C. Huang, H.-Y. Chang, Y.-C. Yeh, and C.Y. Tsai, "Hybridization strategies for continuous ant colony optimization and particle swarm optimization applied to data clustering," Applied Soft Computing Journal, vol. 13, no. 9, pp. 3864-3872, 2013.

[132] R. Rahmani, R. Yusof, M. Seyedmahmoudian, and S. Mekhilef, "Hybrid technique of ant colony and particle swarm optimization for short term wind energy forecasting," Journal of Wind Engineering and Industrial Aerodynamics, vol. 123, pp. 163-170, 2013.

[133] W. Elloumi, N. Baklouti, A. Abraham, and A. M. Alimi, "The multi-objective hybridization of particle swarm optimization and fuzzy ant colony optimization," Journal of Intelligent and Fuzzy Systems, vol. 27, no. 1, pp. 515-525, 2014.

[134] S. M. Sait, A. T. Sheikh, and A. H. El-Maleh, "Cell assignment in hybrid CMOS/nanodevices architecture using a PSO/SA hybrid algorithm," Journal of Applied Research and Technology, vol. 11, no. 5, pp. 653-664, 2013.

[135] H. Jiang and L. Zou, "A hybrid PSO-SA optimizing approach for SVM models in classification," International Journal of Biomathematics, vol. 6, no. 5, Article ID 1350036, 18 pages, 2013.

[136] T. Niknam, M. R. Narimani, and M. Jabbari, "Dynamic optimal power flow using hybrid particle swarm optimization and simulated annealing," International Transactions on Electrical Energy Systems, vol. 23, no. 7, pp. 975-1001, 2013.

[137] F. Khoshahval, A. Zolfaghari, H. Minuchehr, and M. R. Abbasi, "A new hybrid method for multi-objective fuel management optimization using parallel PSO-SA," Progress in Nuclear Energy, vol. 76, pp. 112-121, 2014.

[138] H. Du, P. H. Lou, and W. H. Ye, "Application of hybrid particle swarm optimization in resource constrained multiproject scheduling," Journal of the Chinese Society of Mechanical Engineers, vol. 35, no. 5, pp. 371-379, 2014.

[139] Y. Zhang, S. Wang, Y. Sun, G. Ji, P. Phillips, and Z. Dong, "Binary structuring elements decomposition based on an improved recursive dilation-union model and RSAPSO method," Mathematical Problems in Engineering, vol. 2014, Article ID 272496, 12 pages, 2014.

[140] J. Geng, M.-W. Li, Z.-H. Dong, and Y.-S. Liao, "Port throughput forecasting by MARS-RSVR with chaotic simulated annealing particle swarm optimization algorithm," Neurocomputing, vol. 147, pp. 239-250, 2015.

[141] M. El-Abd, "Testing a particle swarm optimization and artificial bee colony hybrid algorithm on the CEC13 benchmarks," in Proceedings of the IEEE Congress on Evolutionary Computation (CEC '13), pp. 2215-2220, IEEE, Cancún, Mexico, June 2013.

[142] T. K. Sharma, M. Pant, and A. Abraham, "Blend of local and global variant of PSO in ABC," in Proceedings of the World Congress on Nature and Biologically Inspired Computing (NaBIC '13), pp. 113-119, August 2013.

[143] M. S. Kiran and M. Gündüz, "A recombination-based hybridization of particle swarm optimization and artificial bee colony algorithm for continuous optimization problems," Applied Soft Computing Journal, vol. 13, no. 4, pp. 2188-2203, 2013.

[144] L. N. Vitorino, S. F. Ribeiro, and C. J. A. Bastos-Filho, "A mechanism based on artificial bee colony to generate diversity in particle swarm optimization," Neurocomputing, vol. 148, pp. 39-45, 2015.

[145] G. Maione and A. Punzi, "Combining differential evolution and particle swarm optimization to tune and realize fractionalorder controllers," Mathematical and Computer Modelling of Dynamical Systems, vol. 19, no. 3, pp. 277-299, 2013.

[146] Y. G. Fu, M. Y. Ding, C. P. Zhou, and H. P. Hu, "Route planning for unmanned aerial vehicle (UAV) on the sea using hybrid differential evolution and quantum-behaved particle swarm optimization," IEEE Transactions on Systems, Man, and Cybernetics: Systems, vol. 43, no. 6, pp. 1451-1465, 2013.

[147] Vasundhara, D. Mandal, R. Kar, and S. P. Ghoshal, "Digital FIR filter design using fitness based hybrid adaptive differential evolution with particle swarm optimization," Natural Computing, vol. 13, no. 1, pp. 55-64, 2014.

[148] X. Yu, J. Cao, H. Shan, L. Zhu, and J. Guo, "An adaptive hybrid algorithm based on particle swarm optimization and differential evolution for global optimization," The Scientific World Journal, vol. 2014, Article ID 215472, 16 pages, 2014.

[149] G.-G. Wang, A. Hossein Gandomi, X.-S. Yang, and A. Hossein Alavi, "A novel improved accelerated particle swarm optimization algorithm for global numerical optimization," Engineering Computations, vol. 31, no. 7, pp. 1198-1220, 2014.

[150] A. Yadav and K. Deep, "An efficient co-swarm particle swarm optimization for non-linear constrained optimization," Journal of Computational Science, vol. 5, no. 2, pp. 258-268, 2014.

[151] W. Xu, Z. Geng, Q. Zhu, and X. Gu, "Optimal grade transition for polyethylene reactors based on simultaneous strategies and trust region particle swarm optimization," Industrial and Engineering Chemistry Research, vol. 52, no. 9, pp. 3363-3372, 2013.

[152] S. R. Mohanty, N. Kishor, and P. K. Ray, "Robust H-infinite loop shaping controller based on hybrid PSO and harmonic search for frequency regulation in hybrid distributed generation system," International Journal of Electrical Power and Energy Systems, vol. 60, pp. 302-316, 2014.

[153] W. Guo, W. Li, Q. Zhang, L. Wang, Q. Wu, and H. Ren, "Biogeography-based particle swarm optimization with fuzzy 
elitism and its applications to constrained engineering problems," Engineering Optimization, vol. 46, no. 11, pp. 1465-1484, 2014.

[154] C. Y. Qiu, C. L. Wang, and X. Q. Zuo, "A novel multi-objective particle swarm optimization with K-means based global best selection strategy," International Journal of Computational Intelligence Systems, vol. 6, no. 5, pp. 822-835, 2013.

[155] W.-J. Chen, W.-C. Su, and Y.-L. Yang, "Application of constrained multi-objective hybrid quantum particle swarm optimization for improving performance of an ironless permanent magnet linear motor," Applied Mathematics \& Information Sciences, vol. 8, no. 6, pp. 3111-3120, 2014.

[156] A. Ghanei, E. Assareh, M. Biglari, A. Ghanbarzadeh, and A. R. Noghrehabadi, "Thermal-economic multi-objective optimization of shell and tube heat exchanger using particle swarm optimization (PSO)," Heat and Mass Transfer, vol. 50, no. 10, pp. 1375-1384, 2014.

[157] C. Duan, X. Wang, S. Shu, C. Jing, and H. Chang, "Thermodynamic design of Stirling engine using multi-objective particle swarm optimization algorithm," Energy Conversion and Management, vol. 84, pp. 88-96, 2014.

[158] M. R. Amiryousefi, M. Mohebbi, F. Khodaiyan, and M. G. Ahsaee, "Multi-objective optimization of deep-fat frying of ostrich meat plates using multi-objective particle swarm optimization (MOPSO)," Journal of Food Processing and Preservation, vol. 38, no. 4, pp. 1472-1479, 2014.

[159] S. Ganguly, "Multi-objective planning for reactive power compensation of radial distribution networks with unified power quality conditioner allocation using particle swarm optimization," IEEE Transactions on Power Systems, vol. 29, no. 4, pp. 1801-1810, 2014.

[160] E. Z. Zhang, Y. F. Wu, and Q. W. Chen, "A practical approach for solving multi-objective reliability redundancy allocation problems using extended bare-bones particle swarm optimization," Reliability Engineering and System Safety, vol. 127, pp. 65-76, 2014.

[161] R. Perera, E. Sevillano, A. Arteaga, and A. de Diego, "Identification of intermediate debonding damage in FRP-plated RC beams based on multi-objective particle swarm optimization without updated baseline model," Composites Part B: Engineering, vol. 62, pp. 205-217, 2014.

[162] S. Cheng, M.-Y. Chen, and P. J. Fleming, "Improved multiobjective particle swarm optimization with preference strategy for optimal DG integration into the distribution system," Neurocomputing, vol. 148, pp. 23-29, 2015.

[163] M. Daneshyari and G. G. Yen, "Constrained multiple-swarm particle swarm optimization within a cultural framework," IEEE Transactions on Systems, Man, and Cybernetics Part A:Systems and Humans, vol. 42, no. 2, pp. 475-490, 2012.

[164] M. H. Afshar, "Extension of the constrained particle swarm optimization algorithm to optimal operation of multireservoirs system," International Journal of Electrical Power \& Energy Systems, vol. 51, pp. 71-81, 2013.

[165] G. Koulinas, L. Kotsikas, and K. Anagnostopoulos, "A particle swarm optimization based hyper-heuristic algorithm for the classic resource constrained project scheduling problem," Information Sciences, vol. 277, pp. 680-693, 2014.

[166] J. J. Shan and Y. Ren, "Low-thrust trajectory design with constrained particle swarm optimization," Aerospace Science and Technology, vol. 36, pp. 114-124, 2014.

[167] C. C. Yeh and Y. C. Chien, "A particle swarm optimization-like algorithm for constrained minimal spanning tree problems,"
Journal of Marine Science and Technology, vol. 22, no. 3, pp. 341351, 2014.

[168] N. Singh, R. Arya, and R. K. Agrawal, "A novel approach to combine features for salient object detection using constrained particle swarm optimization," Pattern Recognition, vol. 47, no. 4, pp. 1731-1739, 2014.

[169] P. Paliwal, N. P. Patidar, and R. K. Nema, "Determination of reliability constrained optimal resource mix for an autonomous hybrid power system using Particle Swarm Optimization," Renewable Energy, vol. 63, pp. 194-204, 2014.

[170] L. Z. Cui, Z. Ling, J. Poon et al., "A parallel model of independent component analysis constrained by a 5 -parameter reference curve and its solution by multi-target particle swarm optimization," Analytical Methods, vol. 6, no. 8, pp. 2679-2686, 2014.

[171] Y. Y. Shou, Y. Li, and C. T. Lai, "Hybrid particle swarm optimization for preemptive resource-constrained project scheduling," Neurocomputing, vol. 148, pp. 122-128, 2015.

[172] M. Chen and S. A. Ludwig, "Discrete particle swarm optimization with local search strategy for rule classification," in Proceedings of the 4th World Congress on Nature and Biologically Inspired Computing (NaBIC '12), pp. 162-167, November 2012.

[173] M. Shen, Z.-H. Zhan, W.-N. Chen, Y.-J. Gong, J. Zhang, and Y. Li, "Bi-velocity discrete particle swarm optimization and its application to multicast routing problem in communication networks," IEEE Transactions on Industrial Electronics, vol. 61, no. 12, pp. 7141-7151, 2014.

[174] C.-L. Chen, S.-Y. Huang, Y.-R. Tzeng, and C.-L. Chen, "A revised discrete particle swarm optimization algorithm for permutation flow-shop scheduling problem," Soft Computing, vol. 18, no. 11, pp. 2271-2282, 2014.

[175] Q. Cai, M. Gong, B. Shen, L. Ma, and L. Jiao, "Discrete particle swarm optimization for identifying community structures in signed social networks," Neural Networks, vol. 58, pp. 4-13, 2014.

[176] A. H. Kashan, B. Karimi, and A. Noktehdan, "A novel discrete particle swarm optimization algorithm for the manufacturing cell formation problem," The International Journal of Advanced Manufacturing Technology, vol. 73, no. 9-12, pp. 1543-1556, 2014.

[177] N. X. Xu, J. S. Gao, and X. G. Feng, "Study on the optimal design of frequency selective surfaces based on the discrete particle swarm optimization," Acta Physica Sinica, vol. 63, no. 13, Article ID 138401, 2014.

[178] R. Garg and A. K. Singh, "Multi-objective workflow grid scheduling using $\mathcal{\varepsilon}$-fuzzy dominance sort based discrete particle swarm optimization," Journal of Supercomputing, vol. 68, no. 2, pp. 709-732, 2013.

[179] X. Zong, S. Xiong, and Z. Fang, "A conflict-congestion model for pedestrian-vehicle mixed evacuation based on discrete particle swarm optimization algorithm," Computers and Operations Research, vol. 44, pp. 1-12, 2014.

[180] R. Ezzeldin, B. Djebedjian, and T. Saafan, "Integer discrete particle swarm optimization of water distribution networks," Journal of Pipeline Systems Engineering and Practice, vol. 5, no. 1, article 154, 2014.

[181] T. T. Zhai and Z. F. He, "Instance selection for time series classification based on immune binary particle swarm optimization," Knowledge-Based Systems, vol. 49, pp. 106-115, 2013.

[182] K. N. V. D. Sarath and V. Ravi, "Association rule mining using binary particle swarm optimization," Engineering Applications of Artificial Intelligence, vol. 26, no. 8, pp. 1832-1840, 2013. 
[183] M. A. Taha and D. I. Abu Al Nadi, "Spectrum sensing for cognitive radio using binary particle swarm optimization," Wireless Personal Communications, vol. 72, no. 4, pp. 2143-2153, 2013.

[184] A. H. El-Maleh, A. T. Sheikh, and S. M. Sait, "Binary particle swarm optimization (BPSO) based state assignment for area minimization of sequential circuits," Applied Soft Computing Journal, vol. 13, no. 12, pp. 4832-4840, 2013.

[185] A. Erturk, M. K. Gullu, D. Cesmeci, D. Gercek, and S. Erturk, "Spatial resolution enhancement of hyperspectral images using unmixing and binary particle swarm optimization," IEEE Geoscience and Remote Sensing Letters, vol. 11, no. 12, pp. 2100-2104, 2014.

[186] Y. Zhang, S. Wang, P. Phillips, and G. Ji, "Binary PSO with mutation operator for feature selection using decision tree applied to spam detection," Knowledge-Based Systems, vol. 64, pp. 22-31, 2014.

[187] H. T. Yin, P. Fu, and Z. Sun, "Face feature selection and recognition using separability criterion and binary particle swarm optimization algorithm," Chinese Journal of Electronics, vol. 23, no. 2, pp. 361-365, 2014.

[188] J. Yang, H. Zhang, Y. Ling, C. Pan, and W. Sun, “Task allocation for wireless sensor network using modified binary particle swarm optimization," IEEE Sensors Journal, vol. 14, no. 3, pp. 882-892, 2014.

[189] M. R. Ganesh, R. Krishna, K. Manikantan, and S. Ramachandran, "Entropy based Binary Particle Swarm Optimization and classification for ear detection," Engineering Applications of Artificial Intelligence, vol. 27, pp. 115-128, 2014.

[190] A. R. Jordehi and J. Jasni, "Parameter selection in particle swarm optimisation: a survey," Journal of Experimental and Theoretical Artificial Intelligence, vol. 25, no. 4, pp. 527-542, 2013.

[191] S. Kumar and D. K. Chaturvedi, "Tuning of particle swarm optimization parameter using fuzzy logic," in Proceedings of the International Conference on Communication Systems and Network Technologies (CSNT '11), pp. 174-179, June 2011.

[192] W. Zhang, Y. Jin, X. Li, and X. Zhang, "A simple way for parameter selection of standard particle swarm optimization," in Artificial Intelligence and Computational Intelligence Part III, H. Deng, D. Miao, J. Lei, and F. L. Wang, Eds., pp. 436-443, Springer, Berlin, Germany, 2011.

[193] H. Yang, "Particle swarm optimization with modified velocity strategy," in Proceedings of the International Conference on Energy and Environmental Science (ICEES '11), X. Zhou, Ed., pp. 1074-1079, Elsevier Science, 2011.

[194] J. Sun, W. Fang, X. Wu, V. Palade, and W. Xu, "Quantumbehaved particle swarm optimization: analysis of individual particle behavior and parameter selection," Evolutionary Computation, vol. 20, no. 3, pp. 349-393, 2012.

[195] I. M. Yassin, M. N. Taib, R. Adnan, M. K. M. Salleh, and M. K. Hamzah, "Effect of swarm size parameter on Binary Particle Swarm optimization-based NARX structure selection," in Proceedings of the IEEE Symposium on Industrial Electronics and Applications (ISIEA '12), pp. 219-223, Bandung City West Java, Indonesia, September 2012.

[196] D.-S. Wang, H.-J. Wang, and J.-K. Zhang, "Selection of the PSO parameters for inverting of ellipsometry," in Proceedings of the International Conference on Industrial Control and Electronics Engineering (ICICEE 2012), pp. 776-780, August 2012.

[197] H. Hao, N. Hu, X. Xu, and W. Q. Ying, "Parameters selection and optimization of particle swarm optimization algorithm based on molecular force model," in Measurement Technology and Engineering Researches in Industry, P. Yarlagadda and Y. H. Kim, Eds., Parts 1-3, pp. 1370-1373, Trans Tech Publications, Stafa, Switzerland, 2013.

[198] G. Xu, "An adaptive parameter tuning of particle swarm optimization algorithm," Applied Mathematics and Computation, vol. 219, no. 9, pp. 4560-4569, 2013.

[199] P. Chauhan, K. Deep, and M. Pant, "Novel inertia weight strategies for particle swarm optimization," Memetic Computing, vol. 5, no. 3, pp. 229-251, 2013.

[200] W. Zhang, D. Ma, J.-J. Wei, and H.-F. Liang, "A parameter selection strategy for particle swarm optimization based on particle positions," Expert Systems with Applications, vol. 41, no. 7, pp. 3576-3584, 2014.

[201] M. Kanemasa and E. Aiyoshi, "Algorithm tuners for PSO methods and genetic programming techniques for learning tuning rules," IEEJ Transactions on Electrical and Electronic Engineering, vol. 9, no. 4, pp. 407-414, 2014.

[202] K. Wang and J. H. Shen, "The convergence basis of particle swarm optimization," in Proceedings of the International Conference on Industrial Control and Electronics Engineering (ICICEE '12), pp. 63-66, August 2012.

[203] J. Sun, X. Wu, V. Palade, W. Fang, C.-H. Lai, and W. Xu, "Convergence analysis and improvements of quantum-behaved particle swarm optimization," Information Sciences, vol. 193, pp. 81-103, 2012.

[204] T. Kurihara and K. Jin'no, "Analysis of convergence property of PSO and its application to nonlinear blind source separation," in Proceedings of the IEEE Congress on Evolutionary Computation (CEC '13), pp. 976-981, IEEE, Cancun, Mexico, June 2013.

[205] C.-C. Lin, "Dynamic router node placement in wireless mesh networks: a PSO approach with constriction coefficient and its convergence analysis," Information Sciences, vol. 232, pp. 294308, 2013.

[206] Y. Zhang, D.-W. Gong, X.-Y. Sun, and N. Geng, "Adaptive bare-bones particle swarm optimization algorithm and its convergence analysis," Soft Computing, vol. 18, no. 7, pp. 13371352, 2014.

[207] W. T. Lin, Z. Lian, X. Gu, and B. Jiao, "A local and global search combined particle swarm optimization algorithm and its convergence analysis," Mathematical Problems in Engineering, vol. 2014, Article ID 905712, 11 pages, 2014.

[208] S. Kim and L. Li, "Statistical identifiability and convergence evaluation for nonlinear pharmacokinetic models with particle swarm optimization," Computer Methods and Programs in Biomedicine, vol. 113, no. 2, pp. 413-432, 2014.

[209] M. Waintraub, R. Schirru, and C. M. N. A. Pereira, "Multiprocessor modeling of parallel Particle Swarm Optimization applied to nuclear engineering problems," Progress in Nuclear Energy, vol. 51, no. 6, pp. 680-688, 2009.

[210] S.-C. Yu, "Elucidating multiprocessors flow shop scheduling with dependent setup times using a twin particle swarm optimization," Applied Soft Computing Journal, vol. 21, pp. 578589, 2014.

[211] Y. K. Hung and W. C. Wang, "Accelerating parallel particle swarm optimization via GPU," Optimization Methods and Software, vol. 27, no. 1, pp. 33-51, 2012.

[212] B. Rymut, B. Kwolek, and T. Krzeszowski, "GPU-accelerated human motion tracking using particle filter combined with PSO," in Advanced Concepts for Intelligent Vision Systems, ACIVS 2013, J. Blanc-Talon, A. Kasinski, W. Philips, D. Popescu, 
and P. Scheunders, Eds., pp. 426-437, Springer, Berlin, Germany, 2013.

[213] J. Kumar, L. Singh, and S. Paul, "GPU based parallel cooperative particle swarm optimization using C-CUDA: a case study," in Proceedings of the IEEE International Conference on Fuzzy Systems (FUZZ-IEEE '13), July 2013.

[214] O. Awwad, A. Al-Fuqaha, G. Ben Brahim, B. Khan, and A. Rayes, "Distributed topology control in large-scale hybrid RF/FSO networks: SIMT GPU-based particle swarm optimization approach," International Journal of Communication Systems, vol. 26, no. 7, pp. 888-911, 2013.

[215] R.-B. Chen, Y.-W. Hsu, Y. Hung, and W. Wang, "Discrete particle swarm optimization for constructing uniform design on irregular regions," Computational Statistics \& Data Analysis, vol. 72, pp. 282-297, 2014.

[216] J. Liu, X. G. Luo, and X. M. F. Zhang, "Job scheduling algorithm for cloud computing based on particle swarm optimization," in Nanotechnology and Precision Engineering, Pts 1 and 2, Z. Y. Jiang and Y. H. Kim, Eds., pp. 957-960, Trans Tech Publications, Stäfa, Switzerland, 2013.

[217] Y. J. Xu and T. You, "Minimizing thermal residual stresses in ceramic matrix composites by using Iterative Map Reduce guided particle swarm optimization algorithm," Composite Structures, vol. 99, pp. 388-396, 2013.

[218] F. Ramezani, J. Lu, and F. Hussain, "Task scheduling optimization in cloud computing applying multi-objective particle swarm optimization," in Service-Oriented Computing, S. Basu, C. Pautasso, L. Zhang, and X. Fu, Eds., vol. 8274 of Lecture Notes in Computer Science, pp. 237-251, Springer, Berlin, Germany, 2013.

[219] K. Govindarajan, T. S. Somasundaram, and V. S. Kumar, "Particle swarm optimization (PSO)-based clustering for improving the quality of learning using cloud computing," in Proceedings of the IEEE 13th International Conference on Advanced Learning Technologies (ICALT '13), pp. 495-497, IEEE, Beijing, China, July 2013.

[220] F. Ramezani, J. Lu, and F. K. Hussain, "Task-based system load balancing in cloud computing using particle swarm optimization," International Journal of Parallel Programming, vol. 42, no. 5, pp. 739-754, 2014.

[221] S. Ganguly, N. C. Sahoo, and D. Das, "Multi-objective particle swarm optimization based on fuzzy-Pareto-dominance for possibilistic planning of electrical distribution systems incorporating distributed generation," Fuzzy Sets and Systems, vol. 213, pp. 47-73, 2013.

[222] S. Komsiyah, "Computational methods of Gaussian particle swarm optimization (GPSO) and lagrange multiplier on economic dispatch issues (case study on electrical system of JavaBali IV area)," in Proceedings of the International Conference on Advances Science and Contemporary Engineering (ICASCE '13), vol. 68, 2014.

[223] J. W. Feng, F. Tian, P. Jia, Q. He, Y. Shen, and S. Fan, "Improving the performance of electronic nose for wound infection detection using orthogonal signal correction and particle swarm optimization," Sensor Review, vol. 34, no. 4, pp. 389-395, 2014.

[224] E. Pekşen, T. Yas, and A. Kıyak, "1-D DC resistivity modeling and interpretation in anisotropic media using particle swarm optimization," Pure and Applied Geophysics, vol. 171, no. 9, pp. 2371-2389, 2014.

[225] J. Yang, L. F. He, and S. Y. Fu, "An improved PSO-based charging strategy of electric vehicles in electrical distribution grid," Applied Energy, vol. 128, pp. 82-92, 2014.
[226] I. M. de Mendonça, I. C. S. Junior, and A. L. M. Marcato, "Static planning of the expansion of electrical energy transmission systems using particle swarm optimization," International Journal of Electrical Power and Energy Systems, vol. 60, pp. 234-244, 2014.

[227] T.-D. Liu, T.-E. Fan, G.-F. Shao, J.-W. Zheng, and Y.-H. Wen, "Particle swarm optimization of the stable structure of tetrahexahedral Pt-based bimetallic nanoparticles," Physics Letters A, vol. 378, no. 40, pp. 2965-2972, 2014.

[228] U. Aich and S. Banerjee, "Modeling of EDM responses by support vector machine regression with parameters selected by particle swarm optimization," Applied Mathematical Modelling, vol. 38, no. 11-12, pp. 2800-2818, 2014.

[229] C.-J. Chou, C.-Y. Lee, and C.-C. Chen, "Survey of reservoir grounding system defects considering the performance of lightning protection and improved design based on soil drilling data and the particle swarm optimization technique," IEEJ Transactions on Electrical and Electronic Engineering, vol. 9, no. 6, pp. 605-613, 2014.

[230] B. A. Lee, B. S. Kim, M. S. Ko, K. Y. Kim, and S. Kim, "Electrical resistance imaging of two-phase flow with a mesh grouping technique based on particle swarm optimization," Nuclear Engineering and Technology, vol. 46, no. 1, pp. 109-116, 2014.

[231] P. Thakral and A. K. Bakhshi, "Computational atomistic blueprinting of novel conducting copolymers using particle swarm optimization," Journal of Computer-Aided Molecular Design, vol. 28, no. 2, pp. 111-122, 2014.

[232] I. Fister, X.-S. Yang, K. Ljubič, D. Fister, and J. Brest, “Towards the novel reasoning among particles in PSO by the use of RDF and SPARQL," The Scientific World Journal, vol. 2014, Article ID 121782, 10 pages, 2014.

[233] J. Aghaei, K. M. Muttaqi, A. Azizivahed, and M. Gitizadeh, "Distribution expansion planning considering reliability and security of energy using modified PSO (Particle Swarm Optimization) algorithm," Energy, vol. 65, pp. 398-411, 2014.

[234] A. Selakov, D. Cvijetinović, L. Milović, S. Mellon, and D. Bekut, "Hybrid PSO-SVM method for short-term load forecasting during periods with significant temperature variations in city of Burbank," Applied Soft Computing Journal, vol. 16, pp. 80-88, 2014.

[235] Y. Shirvany, Q. Mahmood, F. Edelvik, S. Jakobsson, A. Hedstrom, and M. Persson, "Particle swarm optimization applied to EEG source localization of somatosensory evoked potentials," IEEE Transactions on Neural Systems and Rehabilitation Engineering, vol. 22, no. 1, pp. 11-20, 2014.

[236] D. H. Tungadio, B. P. Numbi, M. W. Siti, and A. A. Jimoh, "Particle swarm optimization for power system state estimation," Neurocomputing, vol. 148, pp. 175-180, 2015.

[237] Y. F. Cai and S. X. Yang, "An improved PSO-based approach with dynamic parameter tuning for cooperative multi-robot target searching in complex unknown environments," International Journal of Control, vol. 86, no. 10, pp. 1720-1732, 2013.

[238] K. Kolomvatsos and S. Hadjieftymiades, "On the use of particle swarm optimization and Kernel density estimator in concurrent negotiations," Information Sciences, vol. 262, pp. 99-116, 2014.

[239] S. K. Pandey, S. R. Mohanty, N. Kishor, and J. P. S. Catalão, "Frequency regulation in hybrid power systems using particle swarm optimization and linear matrix inequalities based robust controller design," International Journal of Electrical Power \& Energy Systems, vol. 63, pp. 887-900, 2014. 
[240] G. Štimac, S. Braut, and R. Žigulić, "Comparative analysis of PSO algorithms for PID controller tuning," Chinese Journal of Mechanical Engineering, vol. 27, no. 5, pp. 928-936, 2014.

[241] N. Nedic, D. Prsic, L. Dubonjic, V. Stojanovic, and V. Djordjevic, "Optimal cascade hydraulic control for a parallel robot platform by PSO," International Journal of Advanced Manufacturing Technology, vol. 72, no. 5-8, pp. 1085-1098, 2014.

[242] W.-D. Chang and C.-Y. Chen, "PID controller design for MIMO processes using improved particle swarm optimization," Circuits, Systems, and Signal Processing, vol. 33, no. 5, pp. 14731490, 2014.

[243] X. Xiang, R. Mutlu, G. Alici, and W. Li, "Control of conducting polymer actuators without physical feedback: simulated feedback control approach with particle swarm optimization," Smart Materials and Structures, vol. 23, no. 3, Article ID 035014, 2014.

[244] K. A. Danapalasingam, "Robust autonomous helicopter stabilizer tuned by particle swarm optimization," International Journal of Pattern Recognition and Artificial Intelligence, vol. 28, no. 1, Article ID 1459002, 2014.

[245] M. J. Mahmoodabadi, M. Taherkhorsandi, and A. Bagheri, "Optimal robust sliding mode tracking control of a biped robot based on ingenious multi-objective PSO," Neurocomputing, vol. 124, pp. 194-209, 2014.

[246] Y. Zhong, X. Huang, P. Meng, and F. Li, "PSO-RBF neural network PID control algorithm of electric gas pressure regulator," Abstract and Applied Analysis, vol. 2014, Article ID 731368, 7 pages, 2014.

[247] J.-W. Perng, G.-Y. Chen, and S.-C. Hsieh, "Optimal pid controller design based on PSO-RBFNN for wind turbine systems," Energies, vol. 7, no. 1, pp. 191-209, 2014.

[248] Y.-C. Huang and Y.-H. Li, "Experiments of iterative learning control system using particle swarm optimization by new bounded constraints on velocity and positioning," Engineering Computations, vol. 31, no. 2, pp. 250-266, 2014.

[249] M. G. Nisha and G. N. Pillai, "Nonlinear model predictive control of MIMO system with relevance vector machines and particle swarm optimization," Control Engineering and Applied Informatics, vol. 16, no. 2, pp. 58-66, 2014.

[250] M. Yousefi, M. Mosalanejad, G. Moradi, and A. Abdipour, "Dual band planar hybrid coupler with enhanced bandwidth using particle swarm optimization technique," IEICE Electronics Express, vol. 9, no. 12, pp. 1030-1035, 2012.

[251] X.-B. Sun, Z.-M. Li, C.-L. Zhao, and Z. Zhou, "Cognitive UWB pulse waveform design based on particle swarm optimization," Ad-Hoc \& Sensor Wireless Networks, vol. 16, no. 1-3, pp. 215-227, 2012.

[252] H. Yongqiang, L. Wentao, and L. Xiaohui, "Particle swarm optimization for antenna selection in MIMO system," Wireless Personal Communications, vol. 68, no. 3, pp. 1013-1029, 2013.

[253] C.-C. Chiu, M.-H. Ho, and S.-H. Liao, "PSO and APSO for optimizing coverage in indoor UWB communication system," International Journal of RF and Microwave Computer-Aided Engineering, vol. 23, no. 3, pp. 300-308, 2013.

[254] A. A. Minasian and T. S. Bird, "Particle swarm optimization of microstrip antennas for wireless communication systems," IEEE Transactions on Antennas and Propagation, vol. 61, no. 12, pp. 6214-6217, 2013.

[255] M. Zubair and M. Moinuddin, "Joint optimization of microstrip patch antennas using particle swarm optimization for UWB systems," International Journal of Antennas and Propagation, vol. 2013, Article ID 649049, 8 pages, 2013.
[256] Y. G. Kim and M. J. Lee, "Scheduling multi-channel and multi-timeslot in time constrained wireless sensor networks via simulated annealing and particle swarm optimization," IEEE Communications Magazine, vol. 52, no. 1, pp. 122-129, 2014.

[257] A. Yazgan and I. Hakki Cavdar, "A comparative study between LMS and PSO algorithms on the optical channel estimation for radio over fiber systems," Optik, vol. 125, no. 11, pp. 2582-2586, 2014.

[258] R. I. Rabady and A. Ababneh, "Global optimal design of optical multilayer thin-film filters using particle swarm optimization," Optik, vol. 125, no. 1, pp. 548-553, 2014.

[259] G. Das, P. K. Pattnaik, and S. K. Padhy, "Artificial Neural Network trained by Particle Swarm Optimization for non-linear channel equalization," Expert Systems with Applications, vol. 41, no. 7, pp. 3491-3496, 2014.

[260] S. Scott-Hayward and E. Garcia-Palacios, "Channel time allocation PSO for gigabit multimedia wireless networks," IEEE Transactions on Multimedia, vol. 16, no. 3, pp. 828-836, 2014.

[261] A. Omidvar and K. Mohammadi, "Particle swarm optimization in intelligent routing of delay-tolerant network routing," EURASIP Journal on Wireless Communications and Networking, vol. 2014, article 147, 8 pages, 2014.

[262] P. Kuila and P. K. Jana, "Energy efficient clustering and routing algorithms for wireless sensor networks: particle swarm optimization approach," Engineering Applications of Artificial Intelligence, vol. 33, pp. 127-140, 2014.

[263] Z. H. Liu and X. L. Wang, "A PSO-based algorithm for load balancing in virtual machines of cloud computing environment," in Advances in Swarm Intelligence: Third International Conference, ICSI 2012, Shenzhen, China, June 17-20, 2012 Proceedings, Part I, Y. Tan, Y. Shi, and Z. Ji, Eds., vol. 7331 of Lecture Notes in Computer Science, pp. 142-147, Springer, Berlin, Germany, 2012.

[264] Z. H. Che, T.-A. Chiang, and Z.-G. Che, "Using analytic network process and turbo particle swarm optimization algorithm for non-balanced supply chain planning considering supplier relationship management," Transactions of the Institute of Measurement and Control, vol. 34, no. 6, pp. 720-735, 2012.

[265] V. Hajipour and S. H. R. Pasandideh, "Proposing an adaptive particle swarm optimization for a novel Bi-objective queuing facility location model," Economic Computation and Economic Cybernetics Studies and Research, vol. 46, no. 3, pp. 223-240, 2012.

[266] F. J. Cabrerizo, E. Herrera-Viedma, and W. Pedrycz, "A method based on PSO and granular computing of linguistic information to solve group decision making problems defined in heterogeneous contexts," European Journal of Operational Research, vol. 230, no. 3, pp. 624-633, 2013.

[267] K. Prescilla and A. I. Selvakumar, "Modified Binary particle swarm optimization algorithm application to real-time task assignment in heterogeneous multiprocessor," Microprocessors and Microsystems, vol. 37, no. 6-7, pp. 583-589, 2013.

[268] H. B. Duan, X. X. Wei, and Z. N. Dong, "Multiple UCAVs cooperative air combat simulation platform based on PSO, ACO, and game theory," IEEE Aerospace and Electronic Systems Magazine, vol. 28, no. 11, pp. 12-19, 2013.

[269] F. Belmecheri, C. Prins, F. Yalaoui, and L. Amodeo, "Particle swarm optimization algorithm for a vehicle routing problem with heterogeneous fleet, mixed backhauls, and time windows," Journal of Intelligent Manufacturing, vol. 24, no. 4, pp. 775-789, 2013.

[270] W. B. Hu, H. Liang, C. Peng, B. Du, and Q. Hu, "A hybrid chaosparticle swarm optimization algorithm for the vehicle routing 
problem with time window," Entropy, vol. 15, no. 4, pp. 12471270, 2013.

[271] A. Al Badawi and A. Shatnawi, "Static scheduling of directed acyclic data flow graphs onto multiprocessors using particle swarm optimization," Computers and Operations Research, vol. 40, no. 10, pp. 2322-2328, 2013.

[272] F. P. Goksal, I. Karaoglan, and F. Altiparmak, "A hybrid discrete particle swarm optimization for vehicle routing problem with simultaneous pickup and delivery," Computers \& Industrial Engineering, vol. 65, no. 1, pp. 39-53, 2013.

[273] W. Zhang, H. Xie, B. Cao, and A. M. K. Cheng, "Energy-aware real-time task scheduling for heterogeneous multiprocessors with particle swarm optimization algorithm," Mathematical Problems in Engineering, vol. 2014, Article ID 287475, 9 pages, 2014.

[274] P. N. Kechagiopoulos and G. N. Beligiannis, "Solving the urban transit routing problem using a particle swarm optimization based algorithm," Applied Soft Computing Journal, vol. 21, pp. 654-676, 2014.

[275] L. Ming, H. Hai, Z. Aimin, S. Yingde, L. Zhao, and Z. Xingguo, "Modeling of mechanical properties of as-cast Mg-Li-Al alloys based on PSO-BP algorithm," China Foundry, vol. 9, no. 2, pp. 119-124, 2012.

[276] J. Q. Chen, Y. Tang, R. Ge, Q. An, and X. Guo, "Reliability design optimization of composite structures based on PSO together with FEA," Chinese Journal of Aeronautics, vol. 26, no. 2, pp. 343-349, 2013.

[277] S. C. Mohan, D. K. Maiti, and D. Maity, "Structural damage assessment using FRF employing particle swarm optimization," Applied Mathematics and Computation, vol. 219, no. 20, pp. 10387-10400, 2013.

[278] J. Chen, Y. Tang, and X. Huang, "Application of surrogate based particle swarm optimization to the reliability-based robust design of composite pressure vessels," Acta Mechanica Solida Sinica, vol. 26, no. 5, pp. 480-490, 2013.

[279] Y. L. Zhang, D. Gallipoli, and C. Augarde, "Parameter identification for elasto-plastic modelling of unsaturated soils from pressuremeter tests by parallel modified particle swarm optimization," Computers and Geotechnics, vol. 48, pp. 293-303, 2013.

[280] G. H. Wang, J. Chen, T. Cai, and B. Xin, "Decompositionbased multi-objective differential evolution particle swarm optimization for the design of a tubular permanent magnet linear synchronous motor," Engineering Optimization, vol. 45, no. 9, pp. 1107-1127, 2013.

[281] T. Lazrag, M. Kacem, P. Dubujet, J. Sghaier, and A. Bellagi, "Determination of unsaturated hydraulic properties using drainage gravity test and particle swarm optimization algorithm," Journal of Porous Media, vol. 16, no. 11, pp. 1025-1034, 2013.

[282] C.-H. Lee, K.-S. Shih, C.-C. Hsu, and T. Cho, "Simulationbased particle swarm optimization and mechanical validation of screw position and number for the fixation stability of a femoral locking compression plate," Medical Engineering \& Physics, vol. 36, no. 1, pp. 57-64, 2014.

[283] J. J. Lake, A. E. Duwel, and R. N. Candler, "Particle swarm optimization for design of slotted MEMS resonators with low thermoelastic dissipation," Journal of Microelectromechanical Systems, vol. 23, no. 2, pp. 364-371, 2014.

[284] A. R. Vosoughi and S. Gerist, "New hybrid FE-PSO-CGAs sensitivity base technique for damage detection of laminated composite beams," Composite Structures, vol. 118, pp. 68-73, 2014.

[285] S. L. M. Ribeiro, T. A. A. Silva, L. M. G. Vieira, T. H. Panzera, K. Boba, and F. Scarpa, "Geometric effects of sustainable auxetic structures integrating the particle swarm optimization and finite element method," Materials Research, vol. 17, no. 3, pp. 747-757, 2014.

[286] P. Kitak, A. Glotic, and I. Ticar, "Heat transfer coefficients determination of numerical model by using particle swarm optimization," IEEE Transactions on Magnetics, vol. 50, no. 2, 2014.

[287] R. Kalatehjari, A. S. A Rashid, N. Ali, and M. Hajihassani, "The contribution of particle swarm optimization to threedimensional slope stability analysis," The Scientific World Journal, vol. 2014, Article ID 973093, 12 pages, 2014.

[288] B. Ashuri and M. Tavakolan, "Fuzzy enabled hybrid genetic algorithm-particle swarm optimization approach to solve TCRO problems in construction project planning," Journal of Construction Engineering and Management, vol. 138, no. 9, pp. 1065-1074, 2012.

[289] A. Bozorgi-Amiri, M. S. Jabalameli, M. Alinaghian, and M. Heydari, "A modified particle swarm optimization for disaster relief logistics under uncertain environment," International Journal of Advanced Manufacturing Technology, vol. 60, no. 14, pp. 357-371, 2012.

[290] J. Sadoghi Yazdi, F. Kalantary, and H. Sadoghi Yazdi, "Calibration of soil model parameters using particle swarm optimization," International Journal of Geomechanics, vol. 12, no. 3, pp. 229-238, 2012.

[291] B. Bolat, O. Altun, and P. Cortés, "A particle swarm optimization algorithm for optimal car-call allocation in elevator group control systems," Applied Soft Computing Journal, vol. 13, no. 5, pp. 2633-2642, 2013.

[292] K. S. J. Babu and D. P. Vijayalakshmi, "Self-adaptive PSOGA hybrid model for combinatorial water distribution network design," Journal of Pipeline Systems Engineering and Practice, vol. 4, no. 1, pp. 57-67, 2013.

[293] S. C. Mohan, A. Yadav, D. Kumar Maiti, and D. Maity, "A comparative study on crack identification of structures from the changes in natural frequencies using GA and PSO," Engineering Computations, vol. 31, no. 7, pp. 1514-1531, 2014.

[294] M. Asadnia, L. H. C. Chua, X. S. Qin, and A. Talei, "Improved particle swarm optimization-based artificial neural network for Rainfall-Runoff modeling," Journal of Hydrologic Engineering, vol. 19, no. 7, pp. 1320-1329, 2014.

[295] B. Yan, S. Goto, A. Miyamoto, and H. Zhao, "Imaging-based rating for corrosion states of weathering steel using wavelet transform and PSO-SVM techniques," Journal of Computing in Civil Engineering, vol. 28, no. 3, Article ID 04014008, 2014.

[296] Y. Wang and L. Li, "A PSO algorithm for constrained redundancy allocation in multi-state systems with bridge topology," Computers \& Industrial Engineering, vol. 68, no. 1, pp. 13-22, 2014.

[297] J. Sadeghi, S. Sadeghi, and S. T. Niaki, "Optimizing a hybrid vendor-managed inventory and transportation problem with fuzzy demand: an improved particle swarm optimization algorithm," Information Sciences, vol. 272, pp. 126-144, 2014.

[298] Ž. Kanović, V. Bugarski, and T. Bačkalić, "Ship lock control system optimization using GA, PSO and ABC: a comparative review," Promet-Traffic \& Transportation, vol. 26, no. 1, pp. 2331, 2014. 
[299] P. Mandal, H. Zareipour, and W. D. Rosehart, "Forecasting aggregated wind power production of multiple wind farms using hybrid wavelet-PSO-NNs," International Journal of Energy Research, vol. 38, no. 13, pp. 1654-1666, 2014.

[300] K. H. Chao, "An extension theory-based maximum power tracker using a particle swarm optimization algorithm," Energy Conversion and Management, vol. 86, pp. 435-442, 2014.

[301] G. G. Chen, L. Liu, P. Song, and Y. Du, "Chaotic improved PSObased multi-objective optimization for minimization of power losses and L index in power systems," Energy Conversion and Management, vol. 86, pp. 548-560, 2014.

[302] C. Hu, G. Jain, P. Zhang, C. Schmidt, P. Gomadam, and T. Gorka, "Data-driven method based on particle swarm optimization and k-nearest neighbor regression for estimating capacity of lithium-ion battery," Applied Energy, vol. 129, pp. 49-55, 2014.

[303] I. Tabet, K. Touafek, N. Bellel, N. Bouarroudj, A. Khelifa, and M. Adouane, "Optimization of angle of inclination of the hybrid photovoltaic-thermal solar collector using particle swarm optimization algorithm," Journal of Renewable and Sustainable Energy, vol. 6, no. 5, Article ID 053116, 2014.

[304] S. Bahrami, R. A. Hooshmand, and M. Parastegari, "Short term electric load forecasting by wavelet transform and grey model improved by PSO (particle swarm optimization) algorithm," Energy, vol. 72, pp. 434-442, 2014.

[305] A. Askarzadeh, "Comparison of particle swarm optimization and other metaheuristics on electricity demand estimation: a case study of Iran," Energy, vol. 72, pp. 484-491, 2014.

[306] M. Sharafi and T. Y. ElMekkawy, "Multi-objective optimal design of hybrid renewable energy systems using PSOsimulation based approach," Renewable Energy, vol. 68, pp. 6779, 2014.

[307] P. García-Triviño, F. Llorens-Iborra, C. A. García-Vázquez, A. J. Gil-Mena, L. M. Fernández-Ramírez, and F. Jurado, "Longterm optimization based on PSO of a grid-connected renewable energy/battery/hydrogen hybrid system," International Journal of Hydrogen Energy, vol. 39, no. 21, pp. 10805-10816, 2014.

[308] S. Biao, H. Chang hua, Y. Xin hua, and H. Chuan, "Mutation particle swarm optimization algorithm for solving the optimal operation model of thermal power plants," Journal of Renewable and Sustainable Energy, vol. 6, no. 4, Article ID 043118, 2014.

[309] J.-H. Chen, H.-T. Yau, and W. Hung, "Design and study on sliding mode extremum seeking control of the chaos embedded particle swarm optimization for maximum power point tracking in wind power systems," Energies, vol. 7, no. 3, pp. 1706-1720, 2014.

[310] M. Seyedmahmoudian, S. Mekhilef, R. Rahmani, R. Yusof, and A. Asghar Shojaei, "Maximum power point tracking of partial shaded photovoltaic array using an evolutionary algorithm: a particle swarm optimization technique," Journal of Renewable and Sustainable Energy, vol. 6, no. 2, Article ID 023102, 2014.

[311] M. M. Aman, G. B. Jasmon, A. H. A. Bakar, and H. Mokhlis, "A new approach for optimum simultaneous multi-DG distributed generation Units placement and sizing based on maximization of system loadability using HPSO (hybrid particle swarm optimization) algorithm," Energy, vol. 66, pp. 202-215, 2014.

[312] C.-L. Xiao and H.-X. Huang, "Optimal design of heating system for rapid thermal cycling mold using particle swarm optimization and finite element method," Applied Thermal Engineering, vol. 64, no. 1-2, pp. 462-470, 2014.

[313] K. L. Lian, J. H. Jhang, and I. S. Tian, "A maximum power point tracking method based on perturb-and-observe combined with particle swarm optimization," IEEE Journal of Photovoltaics, vol. 4, no. 2, pp. 626-633, 2014.

[314] S. N. Qasem and S. M. Shamsuddin, "Radial basis function network based on time variant multi-objective particle swarm optimization for medical diseases diagnosis," Applied Soft Computing Journal, vol. 11, no. 1, pp. 1427-1438, 2011.

[315] Y. Zhang, S. Wang, and L. Wu, "A novel method for magnetic resonance brain image classification based on adaptive chaotic PSO," Progress in Electromagnetics Research, vol. 109, pp. 325343, 2010.

[316] X. D. Guo, C. Wang, and R. G. Yan, "An electromagnetic localization method for medical micro-devices based on adaptive particle swarm optimization with neighborhood search," Measurement, vol. 44, no. 5, pp. 852-858, 2011.

[317] P.-C. Chang, J.-J. Lin, and C.-H. Liu, "An attribute weight assignment and particle swarm optimization algorithm for medical database classifications," Computer Methods and Programs in Biomedicine, vol. 107, no. 3, pp. 382-392, 2012.

[318] L.-F. Chen, C.-T. Su, K.-H. Chen, and P.-C. Wang, "Particle swarm optimization for feature selection with application in obstructive sleep apnea diagnosis," Neural Computing \& Applications, vol. 21, no. 8, pp. 2087-2096, 2012.

[319] W.-T. Sung and Y.-C. Chiang, "Improved particle swarm optimization algorithm for android medical care iot using modified parameters," Journal of Medical Systems, vol. 36, no. 6, pp. 37553763, 2012.

[320] I. Cruz-Aceves, J. G. Aviña-Cervantes, J. M. López-Hernández, and S. E. González-Reyna, "Multiple active contours driven by particle swarm optimization for cardiac medical image segmentation," Computational and Mathematical Methods in Medicine, vol. 2013, Article ID 132953, 13 pages, 2013.

[321] A. Subasi, "Classification of EMG signals using PSO optimized SVM for diagnosis of neuromuscular disorders," Computers in Biology and Medicine, vol. 43, no. 5, pp. 576-586, 2013.

[322] Y. Zhang, S. Wang, G. Ji, and Z. Dong, "An MR brain images classifier system via particle swarm optimization and kernel support vector machine," The Scientific World Journal, vol. 2013, Article ID 130134, 9 pages, 2013.

[323] V. Mangat and R. Vig, "Novel associative classifier based on dynamic adaptive PSO: application to determining candidates for thoracic surgery," Expert Systems with Applications, vol. 41, no. 18, pp. 8234-8244, 2014.

[324] D. Mandal, A. Chatterjee, and M. Maitra, "Robust medical image segmentation using particle swarm optimization aided level set based global fitting energy active contour approach," Engineering Applications of Artificial Intelligence, vol. 35, pp. 199-214, 2014.

[325] Y.-Z. Hsieh, M.-C. Su, and P.-C. Wang, "A PSO-based rule extractor for medical diagnosis," Journal of Biomedical Informatics, vol. 49, pp. 53-60, 2014.

[326] S. Ganapathy, R. Sethukkarasi, P. Yogesh, P. Vijayakumar, and A. Kannan, "An intelligent temporal pattern classification system using fuzzy temporal rules and particle swarm optimization," Academy Proceedings in Engineering Sciences, vol. 39, no. 2, pp. 283-302, 2014.

[327] P. Thakral, V. Arora, S. Kukreti, and A. K. Bakhshi, "Insilico engineering of intrinsically conducting copolymers using particle swarm optimization algorithm," Indian Journal of Chemistry-Section A Inorganic, Physical, Theoretical and Analytical Chemistry, vol. 52, no. 3, pp. 317-326, 2013.

[328] H. Parastar, H. Ebrahimi-Najafabadi, and M. Jalali-Heravi, "Multivariate curve resolution-particle swarm optimization: 
a high-throughput approach to exploit pure information from multi-component hyphenated chromatographic signals," Analytica Chimica Acta, vol. 772, pp. 16-25, 2013.

[329] M. Khajeh, M. Kaykhaii, and A. Sharafi, "Application of PSOartificial neural network and response surface methodology for removal of methylene blue using silver nanoparticles from water samples," Journal of Industrial and Engineering Chemistry, vol. 19, no. 5, pp. 1624-1630, 2013.

[330] Y. Wu, B. Liu, M. Li, and K. Tang, "Prediction of $\mathrm{CO}_{2}$ solubility in polymers by radial basis function artificial neural network based on chaotic self-adaptive particle swarm optimization and fuzzy clustering method," Chinese Journal of Chemistry, vol. 31, no. 12, pp. 1564-1572, 2013.

[331] M. Khajeh and K. Dastafkan, "Removal of molybdenum using silver nanoparticles from water samples: particle swarm optimization-artificial neural network," Journal of Industrial and Engineering Chemistry, vol. 20, no. 5, pp. 3014-3018, 2014.

[332] P. Thakral, V. Kapoor, and A. K. Bakhshi, "In silico architecturing of novel hetero-aromatic bicyclic copolymers using particle swarm optimization algorithm," Indian Journal of Chemistry Section A: Inorganic, Bio-Inorganic, Physical, Theoretical \& Analytical Chemistry, vol. 53, no. 1, pp. 9-16, 2014.

[333] S. Y. Sun and J. W. Li, "Parameter estimation of methanol transformation into olefins through improved particle swarm optimization with attenuation function," Chemical Engineering Research \& Design, vol. 92, no. 11, pp. 2083-2094, 2014.

[334] A. N. Skvortsov, "Estimation of rotation ambiguity in multivariate curve resolution with charged particle swarm optimization (cPSO-MCR)," Journal of Chemometrics, vol. 28, no. 10, pp. 727739, 2014.

[335] M. A. Khansary and A. H. Sani, "Using genetic algorithm (GA) and particle swarm optimization (PSO) methods for determination of interaction parameters in multicomponent systems of liquid-liquid equilibria," Fluid Phase Equilibria, vol. 365, pp. 141-145, 2014.

[336] R. Nasimi and R. Irani, "Identification and modeling of a yeast fermentation bioreactor using hybrid particle swarm optimization-artificial neural networks," Energy Sources, Part A: Recovery, Utilization and Environmental Effects, vol. 36, no. 14, pp. 1604-1611, 2014.

[337] S. Saraswathi, S. Sundaram, N. Sundararajan, M. Zimmermann, and M. Nilsen-Hamilton, "ICGA-PSO-ELM approach for accurate multiclass cancer classification resulting in reduced gene sets in which genes encoding secreted proteins are highly represented," IEEE/ACM Transactions on Computational Biology and Bioinformatics, vol. 8, no. 2, pp. 452-463, 2011.

[338] N. Mansour, F. Kanj, and H. Khachfe, "Particle swarm optimization approach for protein structure prediction in the 3D HP model," Interdisciplinary Sciences: Computational Life Sciences, vol. 4, no. 3, pp. 190-200, 2012.

[339] M. Karabulut and T. Ibrikci, "A Bayesian Scoring Scheme based Particle Swarm Optimization algorithm to identify transcription factor binding sites," Applied Soft Computing Journal, vol. 12, no. 9, pp. 2846-2855, 2012.

[340] Y. J. Liu, X. Shi, G. Huang, B. Li, and L. Zhao, "Classification of diffuse large B cell lymphoma gene expression data based on two-layer particle swarm optimization," in Proceedings of the 10th International Conference on Fuzzy Systems and Knowledge Discovery (FSKD '13), pp. 422-427, Shenyang, China, July 2013.

[341] M. Salahi, A. Jamalian, and A. Taati, "Global minimization of multi-funnel functions using particle swarm optimization,"
Neural Computing and Applications, vol. 23, no. 7-8, pp. 21012106, 2013.

[342] Z. Du, Y. Zhu, and W. Liu, "Combining quantum-behaved PSO and K2 algorithm for enhancing gene network construction," Current Bioinformatics, vol. 8, no. 1, pp. 133-137, 2013.

[343] L.-Y. Chuang, H.-Y. Lane, Y.-D. Lin, M.-T. Lin, C.-H. Yang, and H.-W. Chang, "Identification of SNP barcode biomarkers for genes associated with facial emotion perception using particle swarm optimization algorithm," Annals of General Psychiatry, vol. 13, article 15, 2014.

[344] M. Mandal and A. Mukhopadhyay, "A graph-theoretic approach for identifying non-redundant and relevant gene markers from microarray data using multiobjective binary PSO," PLoS ONE, vol. 9, no. 3, Article ID e90949, 2014.

[345] K.-H. Chen, K.-J. Wang, M.-L. Tsai et al., "Gene selection for cancer identification: a decision tree model empowered by particle swarm optimization algorithm," BMC Bioinformatics, vol. 15, no. 1, article 49, 2014.

[346] P. J. García Nieto, E. García-Gonzalo, J. R. Alonso Fernández, and C. Díaz Muñiz, "Hybrid PSO-SVM-based method for longterm forecasting of turbidity in the Nalón river basin: a case study in Northern Spain," Ecological Engineering, vol. 73, pp. 192-200, 2014.

[347] J. T. Mentzer, W. DeWitt, J. S. Keebler et al., "Defining supply chain management," Journal of Business Logistics, vol. 22, no. 2, pp. 1-25, 2001.

[348] L. Altamirano, M. C. Riff, I. Araya, and L. Trilling, "Anesthesiology nurse scheduling using particle swarm optimization," International Journal of Computational Intelligence Systems, vol. 5, no. 1, pp. 111-125, 2012.

[349] Z. G. Zeng, J. Xu, S. Wu, and M. Shen, "Antithetic method-based particle swarm optimization for a queuing network problem with fuzzy data in concrete transportation systems," ComputerAided Civil and Infrastructure Engineering, vol. 29, no. 10, pp. 771-800, 2014. 


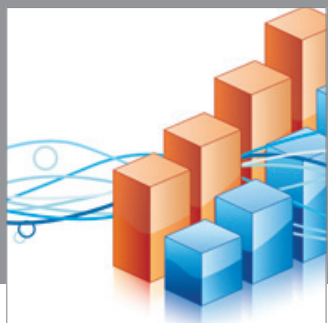

Advances in

Operations Research

mansans

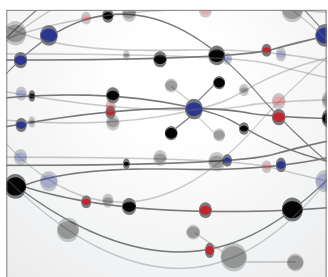

The Scientific World Journal
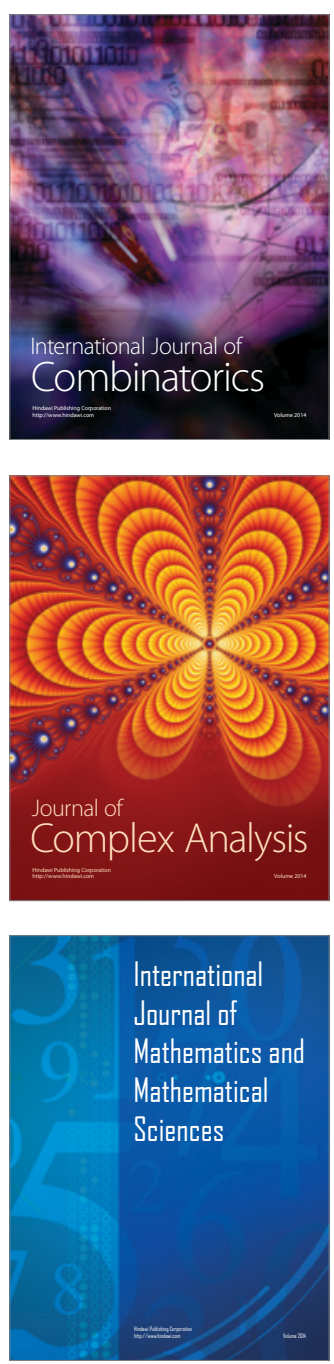
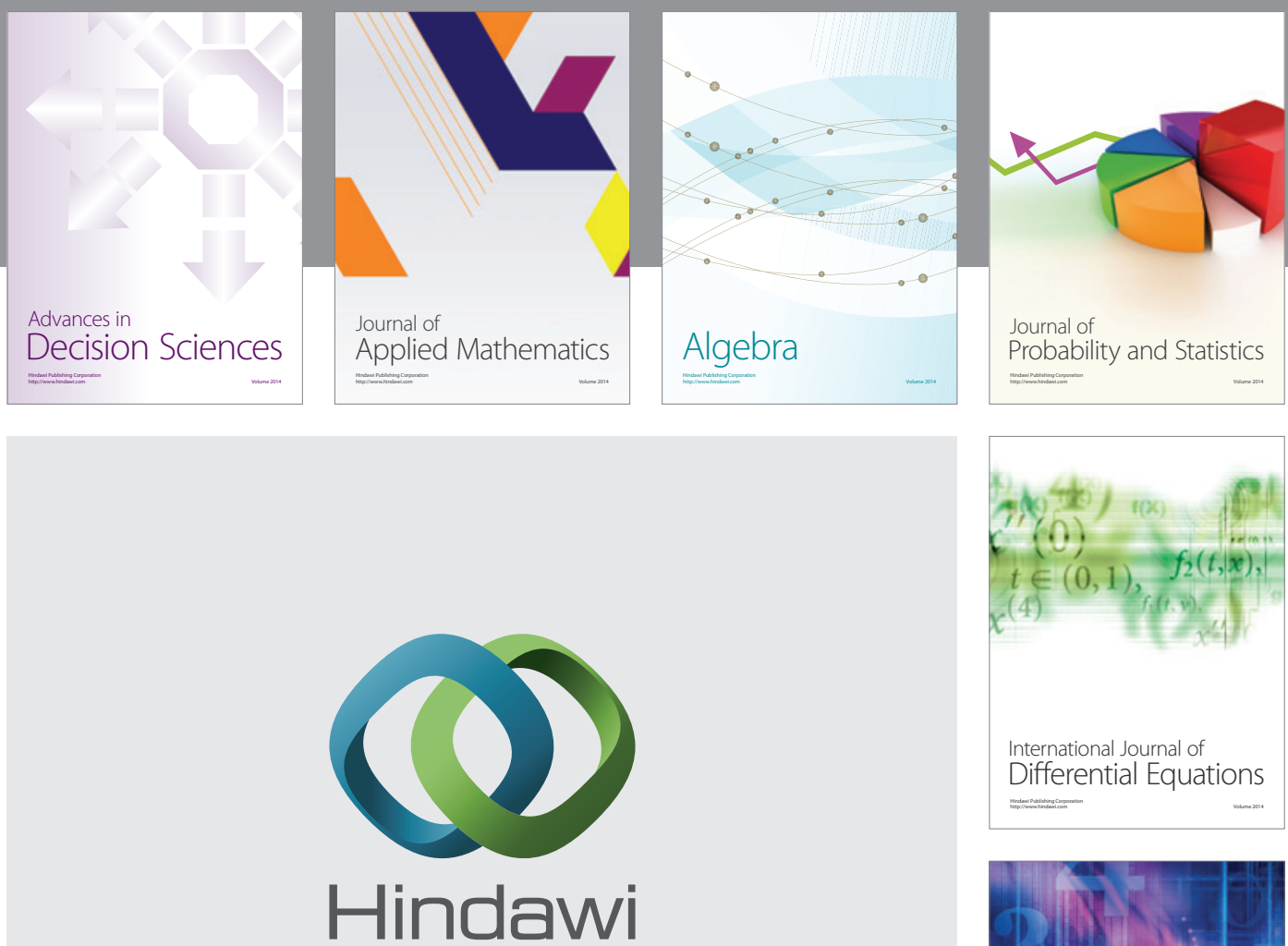

Submit your manuscripts at http://www.hindawi.com
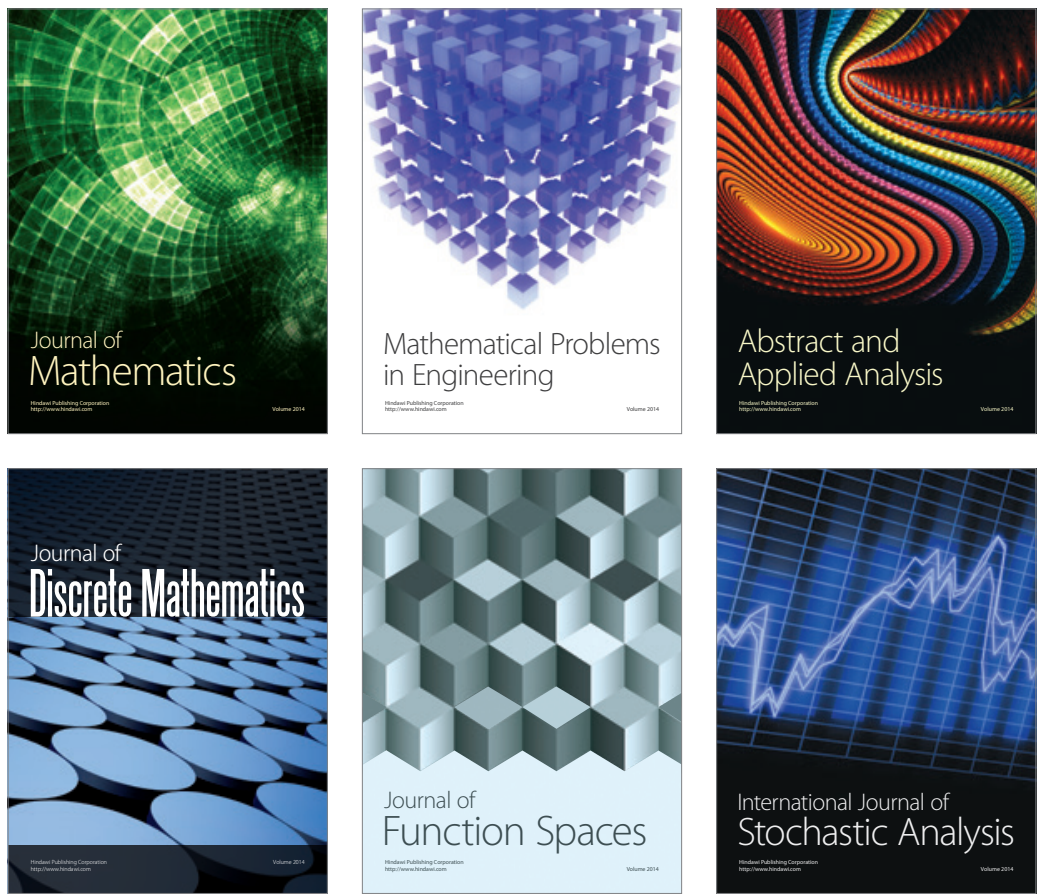

Journal of

Function Spaces

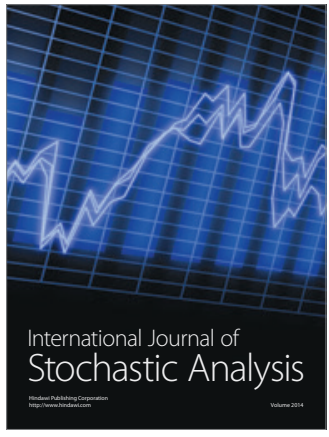

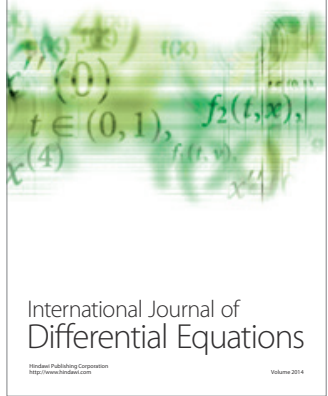
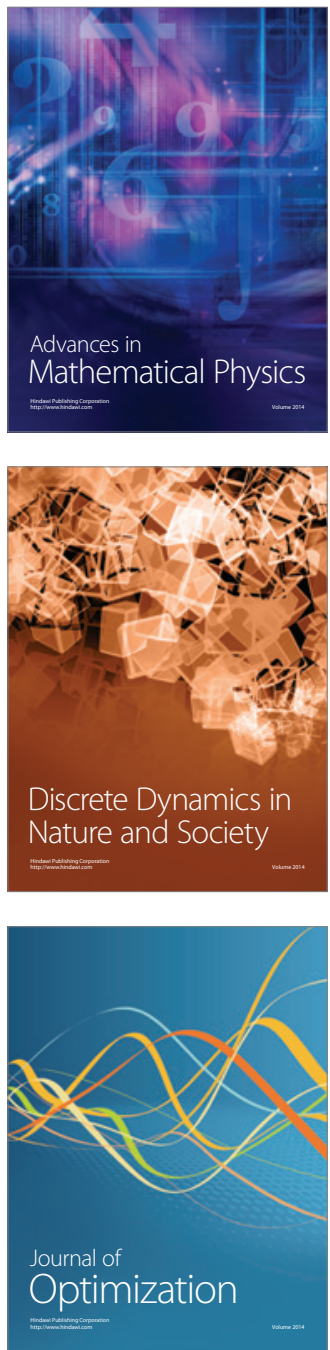